Supporting Information

\title{
Asymmetric Synthesis of Diastereometric Diaminohepatanetetraols. A Proposal for the Configuration of (+)- Zwittermicin A
}

\author{
Evan W. Rogers and Tadeusz F. Molinski* \\ Department of Chemistry and Biochemistry, University of California, San Diego, 9500 \\ Gilman Drive, La Jolla, California 92093, and \\ Department of Chemistry, University of California, Davis, One Shields Avenue, Davis, \\ California 95616 \\ tmolinski@ucsd.edu
}

\section{Materials and Methods}

All non-aqueous reactions were carried out in oven-dried glassware under a nitrogen atmosphere, unless otherwise noted. All solvent were reagent grade. Solvents for dry reactions (DCM, DMF, THF, toluene, acetonitrile, $\mathrm{Et}_{2} \mathrm{O}$ ) were passed through twin alumina columns (J. C. Myer, Glass Contour). DMSO was distilled from calcium hydride under reduced pressure and stored over $4 \AA$ molecular sieves. Dry $\mathrm{MeOH}$ was prepared and stored over $4 \AA$ molecular sieves. Triethylamine and pyridine were distilled from calcium hydride. All other commercially available reagents were used as received. Reactions were monitored by thin layer chromatography (TLC) using 0.25-mm E. Merck per-coated silica gel plates.

NMR spectra were recorded on a Varian Mercury-400 (400 MHz), a Varian Unity-500 (500 MHz) or a Varian Inova-400 (400 MHz) spectrometer. Chemical shifts are reported in parts per million ( $\mathrm{ppm})$ and referenced to residual solvent signal as the internal standard relative $\left[\mathrm{CHCl}_{3}(\delta 7.26)\right.$ or $\mathrm{CD}_{2} \mathrm{HOD}(\delta 3.31)$ for ${ }^{1} \mathrm{H}$, or $\mathrm{CDCl}_{3}(\delta 77.16)$ or $\mathrm{CD}_{3} \mathrm{OD}(\delta 49.0)$ for ${ }^{13} \mathrm{C}$ ] unless otherwise stated. NMR solvents were obtained from Cambridge Isotope Laboratories. Optical rotations were measured using a Jasco DIP-370 digital polarimeter or a Jasco P-1010 polarimeter. IR spectra were obtained on a Mattson Galaxy Series FTIR 3000 or a Nicolet Magna IR 550 spectrometer. Optical rotations for compounds 2-6 were not reported due to being too small for accurate measurements. HRMS were run by either University of California, Riverside mass spectrometry facility, University of California, San Diego mass spectrometry facility or the Scripps Research Institute's Center for Mass Spectrometry. Normal-phase HPLC was carried out on a 
Rainin Rabbit HP systems using $100 \AA \mathrm{SiO}_{2} 10$ x 250 mm column for Microsorb with a UV detector. 


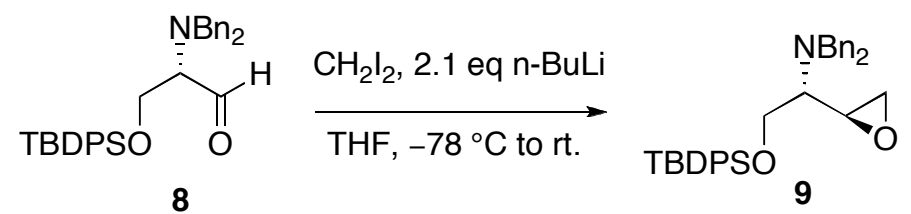

(2S)-2-\{1'(S)-(Dibenzylamino)-2'-[(tert-butyldiphenylsilanyl)oxy]ethyl\}oxirane (9). Under an atmosphere of nitrogen, $n$-BuLi $(3.76 \mathrm{~mL}, 9.41 \mathrm{mmol}, 2.5 \mathrm{M}$ in hexane) was added dropwise to a stirred solution of $(S)$-aldehyde $8(1.60 \mathrm{~g}, 3.15 \mathrm{mmol})$ and $\mathrm{CH}_{2} \mathrm{I}_{2}$ $(0.76 \mathrm{~mL}, 9.41 \mathrm{mmol})$ in anhydrous THF at $-78^{\circ} \mathrm{C}$. The mixture was stirred for $30 \mathrm{~min}$ then warmed to room temperature. The solution was stirred at room temperature for 1 hour then quenched with $10 \mathrm{~mL}$ saturated aqueous $\mathrm{NH}_{4} \mathrm{Cl}$. The mixture was extracted with ethyl ether $(4 \times 15 \mathrm{~mL})$ and combined extracts washed with brine $(20 \mathrm{~mL})$, dried over $\mathrm{Na}_{2} \mathrm{SO}_{4}$ and concentrated under reduced pressure. Flash chromatography on triethylamine-saturated silica (1\% triethylamine in 1:19 EtOAc:hexane) provided 9 (1.34 g, $81 \%$, de $=94 \%$ ) as a light yellow viscous oil: IR (neat) $v 3069,3026,2998,2956$, 2888, 2857, 2803, 1602, 1589, 1493, 1471, 1453, 1428, 1390, 1362, 1253, 1112, 1027, 866, 823, 740, 699, $612 \mathrm{~cm}^{-1} ;[\alpha]_{\mathrm{D}}{ }^{23} 5.6^{\circ}\left(\mathrm{CH}_{2} \mathrm{Cl}_{2}, c 5.64\right) ;{ }^{1} \mathrm{H} \mathrm{NMR}\left(400 \mathrm{MHz}, \mathrm{CDCl}_{3}\right) \delta$ $1.11(\mathrm{~s}, 9 \mathrm{H}), 2.60$ (dd, $J=4.8,2.8 \mathrm{~Hz}, 1 \mathrm{H}), 2.73-2.79(\mathrm{~m}, 2 \mathrm{H}), 3.19(\mathrm{~m}, 1 \mathrm{H}), 3.83-3.98$ $(\mathrm{m}, 6 \mathrm{H}), 7.20-7.50(\mathrm{~m}, 16 \mathrm{H}), 7.71(\mathrm{~d}, J=8.0 \mathrm{~Hz}, 4 \mathrm{H}) ;{ }^{13} \mathrm{C} \mathrm{NMR}\left(100 \mathrm{MHz}, \mathrm{CDCl}_{3}\right) \delta$ $19.3(\mathrm{C}), 27.0\left(\mathrm{CH}_{3}\right), 46.1\left(\mathrm{CH}_{2}\right), 51.3(\mathrm{CH}), 55.3\left(\mathrm{CH}_{2}\right), 60.5(\mathrm{CH}), 61.7\left(\mathrm{CH}_{2}\right), 127.0$ $(\mathrm{CH}), 127.9(\mathrm{CH}), 128.3(\mathrm{CH}), 128.6(\mathrm{CH}), 129.8(\mathrm{CH}), 129.9(\mathrm{CH}), 133.2(\mathrm{C}), 133.4$ (C), $135.7(\mathrm{CH}), 135.8(\mathrm{CH}), 140.3(\mathrm{C})$; HRMS m/z $522.2813[\mathrm{M}+\mathrm{H}]^{+}$, calcd. for $\mathrm{C}_{34} \mathrm{H}_{40} \mathrm{~N}_{1} \mathrm{O}_{2} \mathrm{Si}_{1} 522.2828$.
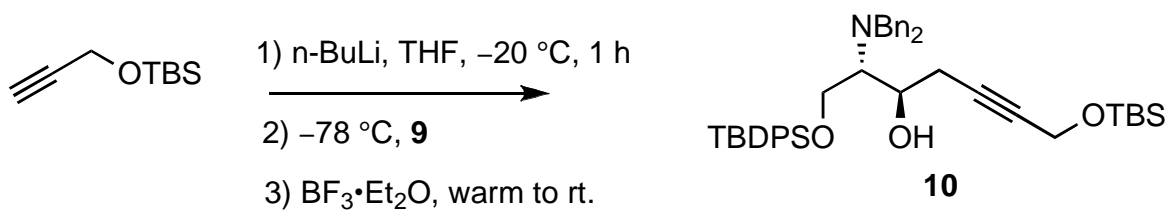

(6S,7R)-6-(dibenzylamino)-2,2,13,13,14,14-hexamethyl-3,3-diphenyl-4,12-dioxa-3,13disilapentadec-9-yn-7-ol (10). Under an atmosphere of nitrogen, $n$-BuLi $(253 \mu \mathrm{L}, 632$ $\mu \mathrm{mol}, 2.5 \mathrm{M}$ in hexane) was added dropwise to a stirred solution of alkyne (118 mg, 690 $\mu \mathrm{mol})$ in anhydrous THF $(1.5 \mathrm{~mL})$ at $-20^{\circ} \mathrm{C}$. The mixture was stirred for 1 hour then cooled to $-78^{\circ} \mathrm{C}$ and epoxide $9(300 \mathrm{mg}, 575 \mu \mathrm{mol}$ in THF $(1.2 \mathrm{~mL}))$ was added dropwise followed by slow addition of $\mathrm{BF}_{3} \cdot \mathrm{Et}_{2} \mathrm{O}(73 \mu \mathrm{L}, 575 \mu \mathrm{mol})$. The mixture was stirred for 1 hour then warmed to room temperature overnight. The solution was quenched with $10 \mathrm{~mL}$ saturated aqueous $\mathrm{NH}_{4} \mathrm{Cl}$, extracted with ethyl ether $(3 \times 20 \mathrm{~mL})$ and combined extracts washed with brine $(20 \mathrm{~mL})$, dried over $\mathrm{Na}_{2} \mathrm{SO}_{4}$ and concentrated under reduced pressure. Flash chromatography (Analogix $4 \mathrm{~g}$ silica cartridge, 1:19 EtOAc:hexane, $13 \mathrm{~mL} / \mathrm{min}$ flow rate) provided $10(283 \mathrm{mg}, 71 \%)$ as a viscous oil: IR (neat) v 3472, 3059, 3018, 2960, 2927, 2853, 1475, 1433, 1359, 1252, 1112, 1079, 831, $691 \mathrm{~cm}^{-1} ;[\alpha]_{\mathrm{D}}^{23}+24.5^{\circ}\left(\mathrm{CHCl}_{3}, c 18.1\right) ;{ }^{1} \mathrm{H} \mathrm{NMR}\left(500 \mathrm{MHz}, \mathrm{CDCl}_{3}\right) \delta 7.72-7.76(\mathrm{~m}$, $4 \mathrm{H}), 7.40-7.49(\mathrm{~m}, 6 \mathrm{H}), 7.20-7.30(\mathrm{~m}, 10 \mathrm{H}), 4.23(\mathrm{~s}, 2 \mathrm{H}), 4.11(\mathrm{dd}, J=11.0,5.0 \mathrm{~Hz}, 1 \mathrm{H})$, $4.06(\mathrm{~m}, 2 \mathrm{H}), 3.87(\mathrm{~d}, J=13.5 \mathrm{~Hz}, 2 \mathrm{H}), 3.58(\mathrm{~d}, J=13.5 \mathrm{~Hz}, 2 \mathrm{H}), 2.79-2.84(\mathrm{~m}, 1 \mathrm{H})$, $2.78(\mathrm{dt}, J=8.0,5.0 \mathrm{~Hz}, 1 \mathrm{H}), 2.74(\mathrm{~d}, J=5.0 \mathrm{~Hz}, 1 \mathrm{H}), 2.29(\mathrm{ddt}, J=17.0,8.0,2.5 \mathrm{~Hz}$, 
1H), $1.10(\mathrm{~s}, 9 \mathrm{H}), 0.91(\mathrm{~s}, 9 \mathrm{H}), 0.11(\mathrm{~s}, 6 \mathrm{H}) ;{ }^{13} \mathrm{C} \mathrm{NMR}\left(100 \mathrm{MHz}, \mathrm{CDCl}_{3}\right) \delta 139.9(\mathrm{C})$, $135.9(\mathrm{CH}), 135.8(\mathrm{CH}), 133.1(\mathrm{C}), 132.9(\mathrm{C}), 130.0(\mathrm{CH}), 129.0(\mathrm{CH}), 128.4(\mathrm{CH})$, $128.0(\mathrm{CH}), 127.9(\mathrm{CH}), 127.1(\mathrm{CH}), 82.4(\mathrm{C}), 81.0(\mathrm{C}), 70.1(\mathrm{CH}), 61.5(\mathrm{CH}), 61.4$ $\left(\mathrm{CH}_{2}\right), 55.4\left(\mathrm{CH}_{2}\right), 52.1\left(\mathrm{CH}_{2}\right), 27.1\left(\mathrm{CH}_{3}\right), 26.0\left(\mathrm{CH}_{3}\right), 25.9\left(\mathrm{CH}_{2}\right), 19.2(\mathrm{C}), 18.5(\mathrm{C}),-$ $5.0\left(\mathrm{CH}_{3}\right)$; HREIMS $\mathrm{m} / z 691.3871[\mathrm{M}]^{+}$, calcd. for $\mathrm{C}_{43} \mathrm{H}_{57} \mathrm{~N}_{1} \mathrm{O}_{3} \mathrm{Si}_{2} 691.3871$.

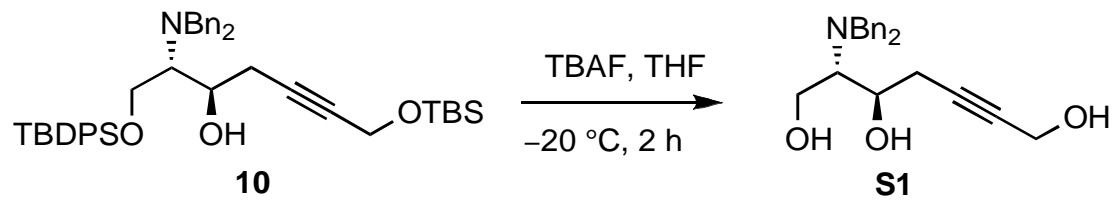

(2S,3R)-2-(dibenzylamino)hept-5-yne-1,3,7-triol (S1). Under an atmosphere of nitrogen TBAF (296 mg, $938 \mu \mathrm{mol}$ ) was added to a stirred solution of alkyne $\mathbf{1 0}$ (270 mg, 390 $\mu \mathrm{mol})$ in THF $(3 \mathrm{~mL})$ at $-20^{\circ} \mathrm{C}$. The mixture was stirred for 2 hours then quenched by addition of saturated aqueous $\mathrm{NH}_{4} \mathrm{Cl}(5 \mathrm{~mL})$. The mixture was extracted with ethyl acetate $(4 \times 3 \mathrm{~mL})$ and combined extracts washed with brine $(5 \mathrm{~mL})$, dried over $\mathrm{Na}_{2} \mathrm{SO}_{4}$ and concentrated under reduced pressure. Flash chromatography (Analogix $4 \mathrm{~g}$ silica cartridge, 1:19 MeOH: $\mathrm{CH}_{2} \mathrm{Cl}_{2}, 12 \mathrm{~mL} / \mathrm{min}$ flow rate) provided the $\mathbf{S 1}(129 \mathrm{mg}, 97 \%$ ) as a viscous oil: IR (neat) $v$ 3355, 2920, 2843, 1499, 1452, 1367, 1134, 1072, $1033 \mathrm{~cm}^{-1}$; $[\alpha]_{\mathrm{D}}{ }^{24}+1.3^{\circ}\left(\mathrm{CHCl}_{3}, c 0.7\right) ;{ }^{1} \mathrm{H}$ NMR $\left(400 \mathrm{MHz}, \mathrm{CDCl}_{3}\right) \delta 7.20-7.35(\mathrm{~m}, 10 \mathrm{H}), 4.11(\mathrm{~s}$, $3 \mathrm{H}), 4.00(\mathrm{dd}, J=11.8,5.2 \mathrm{~Hz}, 1 \mathrm{H}), 3.93(\mathrm{dd}, J=11.8,6.4 \mathrm{~Hz}, 1 \mathrm{H}), 3.80(\mathrm{~d}, J=12.4 \mathrm{~Hz}$, $2 \mathrm{H}), 3.67(\mathrm{~d}, J=12.4 \mathrm{~Hz}, 2 \mathrm{H}), 2.62-2.80(\mathrm{~m}, 2 \mathrm{H}), 2.36(\mathrm{dd}, J=17.2,7.2 \mathrm{~Hz}, 1 \mathrm{H}) ;{ }^{13} \mathrm{C}$ NMR (100 MHz, $\left.\mathrm{CDCl}_{3}\right) \delta 139.5(\mathrm{C}), 129.0(\mathrm{CH}), 128.3(\mathrm{CH}), 127.1(\mathrm{CH}), 82.8(\mathrm{C})$, $81.0(\mathrm{C}), 69.9(\mathrm{CH}), 61.5(\mathrm{CH}), 59.0\left(\mathrm{CH}_{2}\right), 54.7\left(\mathrm{CH}_{2}\right), 50.7\left(\mathrm{CH}_{2}\right), 25.9\left(\mathrm{CH}_{2}\right)$; HREIMS $m / z 339.1824[\mathrm{M}]^{+}$, calcd. for $\mathrm{C}_{21} \mathrm{H}_{25} \mathrm{~N}_{1} \mathrm{O}_{3} 339.1829$.

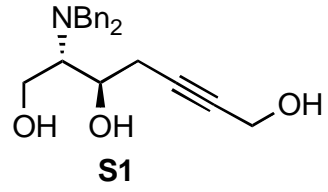

S1
1) $15 \mathrm{~mol} \% \mathrm{CSA}$, acetone dimethoxypropane, $50^{\circ} \mathrm{C}, 2 \mathrm{~h}$

2) 9:2:1 THF : AcOH: $\mathrm{H}_{2} \mathrm{O}$, rt $1 \mathrm{~h}$

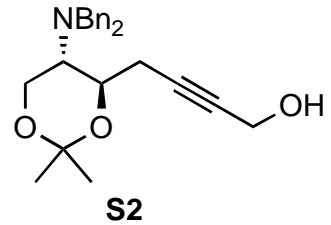

S2

4-((4R,5S)-5-(dibenzylamino)-2,2-dimethyl-1,3-dioxan-4-yl)but-2-yn-1-ol (S2). A sealed vial containing S1 $(44.3 \mathrm{mg}, 130 \mu \mathrm{mol}$, in 1:1 2,2-dimethoxypropane /acetone (2 $\mathrm{mL})$ ) and CSA $(4.5 \mathrm{mg}, 20 \mu \mathrm{mol})$ was heated at $50^{\circ} \mathrm{C}$ with stirring for 2 hours. The stirred mixture was cooled to room temperature and quenched with saturated aqueous $\mathrm{NaHCO}_{3}(5 \mathrm{~mL})$. The mixture was extracted with ethyl ether $(3 \times 5 \mathrm{~mL})$ and combined extracts washed with brine $(5 \mathrm{~mL})$, dried over $\mathrm{Na}_{2} \mathrm{SO}_{4}$ and concentrated under reduced pressure. The crude product was redissolved in $1.5 \mathrm{~mL}$ of $4: 2: 1 \mathrm{THF} / \mathrm{acetic}$ acid/water and stirred for 1 hour at room temperature. The stirred mixture was quenched with saturated aqueous $\mathrm{NaHCO}_{3}(10 \mathrm{~mL})$ extracted with ethyl ether $(3 \times 5 \mathrm{~mL})$ and combined extracts washed with brine $(5 \mathrm{~mL})$, dried over $\mathrm{Na}_{2} \mathrm{SO}_{4}$ and concentrated under reduced pressure. Flash chromatography (Analogix $4 \mathrm{~g}$ silica cartridge, 20\% ethyl acetate in hexane, $12 \mathrm{~mL} / \mathrm{min}$ flow rate) provided $\mathbf{S 2}(399 \mathrm{mg}, 79 \%)$ as a viscous oil: IR (neat) $v$ 3445, 3085, 3060, 3027, 2991, 2935, 2834, 2806, 1949, 1871, 1816, 1602, 1585, 1494, 
1453, 1378, 1245, 1224, 1198, 1161, 1142, 1106, 1057, 1027, 974, 894, 822, 748, 699 $\mathrm{cm}^{-1} ;[\alpha]_{\mathrm{D}}{ }^{23} 8.1^{\mathrm{o}}\left(\mathrm{CHCl}_{3}, c 0.75\right) ;{ }^{1} \mathrm{H}$ NMR $\left(300 \mathrm{MHz}, \mathrm{CDCl}_{3}\right) \delta 1.34(\mathrm{~s}, 3 \mathrm{H}), 1.41(\mathrm{~s}$, $3 \mathrm{H}), 2.38(\mathrm{ddt}, J=17.0,6.6,2.1 \mathrm{~Hz}, 1 \mathrm{H}), 2.71(\mathrm{dq}, J=17.0,2.1 \mathrm{~Hz}, 1 \mathrm{H}), 2.86(\mathrm{dt}, J=$ 9.9, $5.7 \mathrm{~Hz}, 1 \mathrm{H}), 3.52(\mathrm{~d}, J=13.5 \mathrm{~Hz}, 2 \mathrm{H}), 3.86-4.02(\mathrm{~m}, 5 \mathrm{H}), 4.12(\mathrm{~m}, 2 \mathrm{H}), 7.22-7.36$ $(\mathrm{m}, 10 \mathrm{H}) ;{ }^{13} \mathrm{C}$ NMR $\left(75 \mathrm{MHz}, \mathrm{CDCl}_{3}\right) \delta 21.7\left(\mathrm{CH}_{3}\right), 23.3\left(\mathrm{CH}_{2}\right), 26.8\left(\mathrm{CH}_{3}\right), 51.5\left(\mathrm{CH}_{2}\right)$, $54.9\left(\mathrm{CH}_{2}\right), 57.0(\mathrm{CH}), 57.9\left(\mathrm{CH}_{2}\right), 68.9(\mathrm{CH}), 79.8(\mathrm{C}), 83.1(\mathrm{C}), 99.6(\mathrm{C}), 127.3(\mathrm{CH})$, $128.5(\mathrm{CH}), 128.9(\mathrm{CH}), 139.5(\mathrm{C})$; HRMS m/z $380.2212[\mathrm{M}+\mathrm{H}]^{+}$, calcd. for $\mathrm{C}_{24} \mathrm{H}_{30} \mathrm{~N}_{1} \mathrm{O}_{3}$ 380.2226 .

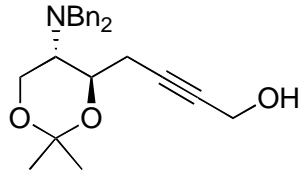

S2

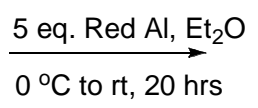

$0^{\circ} \mathrm{C}$ to rt, $20 \mathrm{hrs}$

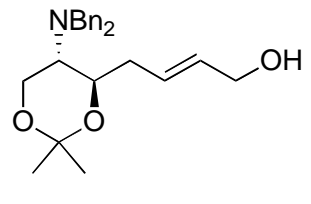

11

(E)-4-((4R,5S)-5-(dibenzylamino)-2,2-dimethyl-1,3-dioxan-4-yl)but-2-en-1-ol (11). Under an atmosphere of nitrogen, Red-Al $65 \mathrm{wt} \%$ in toluene $(87.4 \mu \mathrm{L}, 291 \mu \mathrm{mol})$ was added dropwise to a stirred solution of $\mathbf{S 2}(22 \mathrm{mg}, 58.2 \mu \mathrm{mol})$ in anhydrous ethyl ether $(600 \mu \mathrm{L})$ at $-10{ }^{\circ} \mathrm{C}$. The mixture was allowed to warm to room temperature and stirred overnight. After 20 hours the reaction was cooled to $-10^{\circ} \mathrm{C}$ and quenched by dropwise addition of a 1:3 $\mathrm{H}_{2} 0$ :THF $(300 \mu \mathrm{L})$, warmed to room temperature and added to saturated aqueous $\mathrm{NH}_{4} \mathrm{Cl}(5 \mathrm{~mL})$. The mixture was extracted with ethyl ether $(4 \times 3 \mathrm{~mL})$ and combined extracts washed with brine $(5 \mathrm{~mL})$, dried over $\mathrm{Na}_{2} \mathrm{SO}_{4}$ and concentrated under reduced pressure. Flash chromatography (silica, 30\% ethyl acetate in hexane) provided 5 (17.2 mg, 78\%) as a viscous oil: IR (neat) $v 3432,3060,3026,2990,2938,2835,2807$, $1494,1453,1378,1224,1201,1105,973,745,699 \mathrm{~cm}^{-1} ;[\alpha]_{\mathrm{D}}{ }^{23} 11.5^{\mathrm{o}}\left(\mathrm{CHCl}_{3}, c 1.78\right) ;{ }^{1} \mathrm{H}$ NMR (400 MHz, $\left.\mathrm{CDCl}_{3}\right) \delta 7.22-7.36(\mathrm{bm}, 10 \mathrm{H}), 5.61(\mathrm{dt}, J=15.2,6.4 \mathrm{~Hz}, 1 \mathrm{H}), 5.53(\mathrm{dt}$, $J=15.2,5.2 \mathrm{~Hz}, 1 \mathrm{H}), 4.00-4.10(\mathrm{bm}, 2 \mathrm{H}), 3.80-4.00(\mathrm{~m}, 6 \mathrm{H}), 3.50(\mathrm{~d}, J=13.6 \mathrm{~Hz}, 2 \mathrm{H})$, $2.75(\mathrm{dt}, J=9.6,6.0 \mathrm{~Hz}, 1 \mathrm{H}), 2.59(\mathrm{~m}, 1 \mathrm{H}), 2.08$ (p, $J=7.6 \mathrm{~Hz}, 1 \mathrm{H}, 1.37$ (s, 3H), 1.30 (s, $3 \mathrm{H}) ;{ }^{13} \mathrm{C} \mathrm{NMR}\left(100 \mathrm{MHz}, \mathrm{CDCl}_{3}\right) \delta 139.7(\mathrm{C}), 131.1(\mathrm{CH}), 129.3(\mathrm{CH}), 129.0(\mathrm{CH})$, $128.4(\mathrm{CH}), 127.2(\mathrm{CH}), 99.3(\mathrm{C}), 69.7(\mathrm{CH}), 63.9\left(\mathrm{CH}_{2}\right), 57.9\left(\mathrm{CH}_{2}\right), 57.5(\mathrm{CH}), 54.9$ $\left(\mathrm{CH}_{2}\right), 35.2\left(\mathrm{CH}_{2}\right), 26.7\left(\mathrm{CH}_{3}\right), 21.8\left(\mathrm{CH}_{3}\right) ; \mathrm{HRMS} \mathrm{m} / z 382.2386[\mathrm{M}+\mathrm{H}]^{+}$, calcd. for $\mathrm{C}_{24} \mathrm{H}_{32} \mathrm{~N}_{1} \mathrm{O}_{3} 382.2382$.
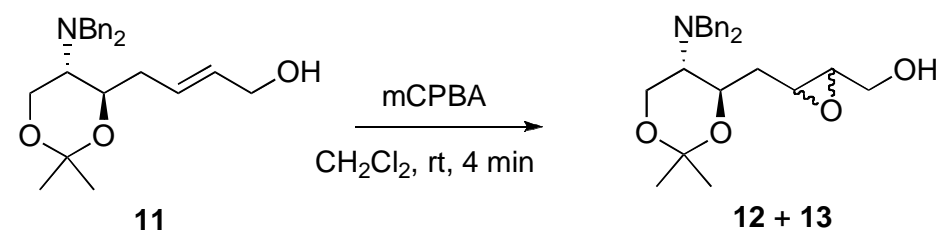

(3-(((4R,5S)-5-(dibenzylamino)-2,2-dimethyl-1,3-dioxan-4-yl)methyl)oxiran-2yl)methanol $(12+13)$. To a solution of $11(4.57 \mathrm{~g}, 12.0 \mathrm{mmol})$ in dichloromethane (66 $\mathrm{mL})$ at room temperature was added $m$-chloroperoxybenzoic acid (1.97 g, $11.4 \mathrm{mmol})$. Solution is stirred for 4 minutes quenched with saturated aqueous $\mathrm{NaHCO}_{3}(200 \mathrm{~mL})$. The aqueous was layer extracted with hexane $(4 \times 100 \mathrm{~mL})$ combined extracts washed with brine $(200 \mathrm{~mL})$, dried over $\mathrm{Na}_{2} \mathrm{SO}_{4}$ and concentrated under reduced pressure. Flash 
chromatography on triethylamine saturated silica $(25 \%, 30 \%$ then $50 \%$ ethyl acetate in hexane) provided an inseparable mixture of $\mathbf{1 2}$ and $\mathbf{1 3}$ (3.12 g, 69\%, 1:1.8 of 12/13 by NMR) as a viscous oil.
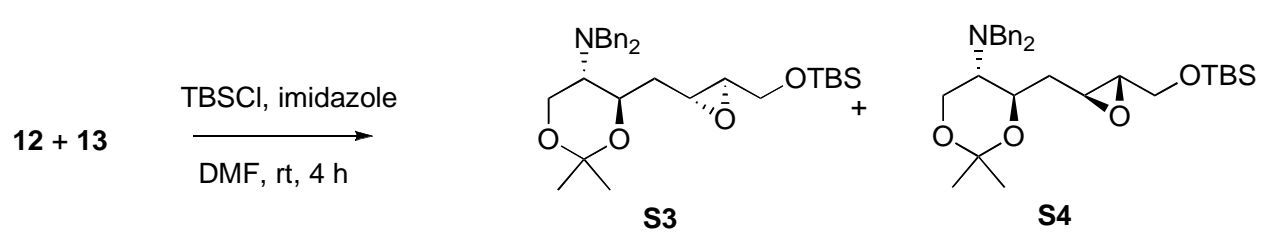

Synthesis of protected epoxides S3 and S4. To a solution of a 1:1 mixture of $\mathbf{1 2}$ and $\mathbf{1 3}$ $(69 \mathrm{mg}, 173 \mu \mathrm{mol})$ in DMF $(1.0 \mathrm{~mL})$ at $0{ }^{\circ} \mathrm{C}$ under nitrogen was added imidazole $(25 \mathrm{mg}$, $347 \mu \mathrm{mol})$ and tert-butylchlorodimethylsilane $(34 \mathrm{mg}, 226 \mu \mathrm{mol})$. The mixture was warmed to room temperature and stirred for 4 hours. The reaction was quenched with 7 $\mathrm{mL}$ water, extracted with ethyl ether $(3 \times 3 \mathrm{~mL})$ and combined extracts washed with brine $(5 \mathrm{~mL})$, dried over $\mathrm{Na}_{2} \mathrm{SO}_{4}$ and concentrated under reduced pressure. Flash chromatography (silica, 3\% ethyl acetate in hexane) provided S3 and S4 (37 mg and 41.2 mg respectively, $88 \%$ ) as viscous oils:

$((4 R, 5 S)-N, N$-dibenzyl-4-(((2R,3R)-3-((tert-butyldimethylsilyloxy)methyl)oxiran-2yl)methyl)-2,2-dimethyl-1,3-dioxan-5-amine (S3). IR (neat) v 3018, 2919, 2853, 1450, $1376,1252,1112,839,782,740 \mathrm{~cm}^{-1} ;[\alpha]_{\mathrm{D}}{ }^{23}+10.5^{\circ}\left(\mathrm{CHCl}_{3}, c 1.82\right) ;{ }^{1} \mathrm{H} \mathrm{NMR}(400$ $\left.\mathrm{MHz}, \mathrm{CDCl}_{3}\right) \delta 7.28-7.32(\mathrm{~m}, 8 \mathrm{H}), 7.20-7.27(\mathrm{~m}, 2 \mathrm{H}), 4.04(\mathrm{td}, J=9.6,2.0 \mathrm{~Hz}, 1 \mathrm{H}), 3.96$ $(\mathrm{dd}, J=12.0,6.4 \mathrm{~Hz}, 1 \mathrm{H}), 3.90(\mathrm{~d}, J=13.6 \mathrm{~Hz}, 2 \mathrm{H}), 3.88(\mathrm{dd}, J=12.0,5.6 \mathrm{~Hz}, 1 \mathrm{H}), 3.79$ (dd, $J=12.0,3.0 \mathrm{~Hz}, 1 \mathrm{H}), 3.57(\mathrm{dd}, J=12.0,4.8 \mathrm{~Hz}, 1 \mathrm{H}), 3.50(\mathrm{~d}, J=13.6 \mathrm{~Hz}, 2 \mathrm{H}), 2.92$ $(\mathrm{m}, 1 \mathrm{H}), 2.81(\mathrm{~m}, 1 \mathrm{H}), 2.69(\mathrm{dt}, J=10.0,5.4 \mathrm{~Hz}, 1 \mathrm{H}), 2.06(\mathrm{ddd}, J=14.4,6.8,2.0 \mathrm{~Hz}$, 1H), 1.48 (ddd, $J=14.4,9.6,4.4 \mathrm{~Hz}, 1 \mathrm{H}), 1.42$ (s, 3H), 1.30 (s, 3H), 0.90 (s, 9H), 0.08 $(\mathrm{s}, 3 \mathrm{H}), 0.07(\mathrm{~s}, 3 \mathrm{H}) ;{ }^{13} \mathrm{C} \mathrm{NMR}\left(100 \mathrm{MHz}, \mathrm{CDCl}_{3}\right) \delta 139.5(\mathrm{C}), 129.0(\mathrm{CH}), 128.5(\mathrm{CH})$, $127.2(\mathrm{CH}), 99.3(\mathrm{C}), 67.7(\mathrm{CH}), 63.7\left(\mathrm{CH}_{2}\right), 59.4(\mathrm{CH}), 58.1\left(\mathrm{CH}_{2}\right), 57.9(\mathrm{CH}), 54.8$ $\left(\mathrm{CH}_{2}\right), 53.3(\mathrm{CH}), 35.6\left(\mathrm{CH}_{2}\right), 27.0\left(\mathrm{CH}_{3}\right), 26.0\left(\mathrm{CH}_{3}\right), 21.6\left(\mathrm{CH}_{3}\right), 18.5(\mathrm{C}),-5.1\left(\mathrm{CH}_{3}\right)$, $5.2\left(\mathrm{CH}_{3}\right)$; HREIMS $m / z 511.3116[\mathrm{M}]^{+}$, calcd. for $\mathrm{C}_{30} \mathrm{H}_{45} \mathrm{~N}_{1} \mathrm{O}_{4} \mathrm{Si}_{1} 511.3112$.

(4R,5S)- $N, N$-dibenzyl-4-(((2S,3S)-3-((tert-butyldimethylsilyloxy)methyl)oxiran-2yl)methyl)-2,2-dimethyl-1,3-dioxan-5-amine (S4). IR (neat) v 3026, 2952, 2926, 2853, $\left.1442,1376,1252,1227,1103,831,773,749,699 \mathrm{~cm}^{-1} ;[\alpha]_{\mathrm{D}}{ }^{24}+6.2^{\circ} \mathrm{CHCl}_{3}, c 2.24\right) ;{ }^{1} \mathrm{H}$ NMR (400 MHz, $\left.\mathrm{CDCl}_{3}\right) \delta$ 7.28-7.34 (m, 8H), 7.20-7.27 (m, 2H), 3.84-3.96 (m, 5H), $3.75(\mathrm{dd}, J=12.0,3.2 \mathrm{~Hz}, 1 \mathrm{H}), 3.57(\mathrm{dd}, J=12.0,5.0 \mathrm{~Hz}, 1 \mathrm{H}), 3.51(\mathrm{~d}, J=14.0 \mathrm{~Hz}, 2 \mathrm{H})$, 2.83-2.88 (m, 2H), $2.76(\mathrm{dt}, J=9.6,6.0 \mathrm{~Hz}, 1 \mathrm{H}), 1.95$ (ddd, $J=14.4,6.0,2.4 \mathrm{~Hz}, 1 \mathrm{H})$, 1.79 (ddd, $J=14.4,8.8,4.4 \mathrm{~Hz}, 1 \mathrm{H}), 1.38(\mathrm{~s}, 3 \mathrm{H}), 1.30$ (s, 3H), 0.89 (s, 9H), 0.07 (s, $3 \mathrm{H}), 0.06(\mathrm{~s}, 3 \mathrm{H}) ;{ }^{13} \mathrm{C} \mathrm{NMR}\left(100 \mathrm{MHz}, \mathrm{CDCl}_{3}\right) \delta 139.5(\mathrm{C}), 128.9(\mathrm{CH}), 128.5(\mathrm{CH})$, $127.3(\mathrm{CH}), 99.2(\mathrm{C}), 67.8(\mathrm{CH}), 63.9\left(\mathrm{CH}_{2}\right), 58.3(\mathrm{CH}), 58.1\left(\mathrm{CH}_{2}\right), 58.0(\mathrm{CH}), 54.8$ $\left(\mathrm{CH}_{2}\right), 53.8(\mathrm{CH}), 34.6\left(\mathrm{CH}_{2}\right), 26.9\left(\mathrm{CH}_{3}\right), 26.0\left(\mathrm{CH}_{3}\right), 21.6\left(\mathrm{CH}_{3}\right), 18.5(\mathrm{C}),-5.1\left(\mathrm{CH}_{3}\right)$, $5.2\left(\mathrm{CH}_{3}\right)$; HREIMS $m / z 511.3107[\mathrm{M}]^{+}$, calcd. for $\mathrm{C}_{30} \mathrm{H}_{45} \mathrm{~N}_{1} \mathrm{O}_{4} \mathrm{Si}_{1} 511.3112$. 

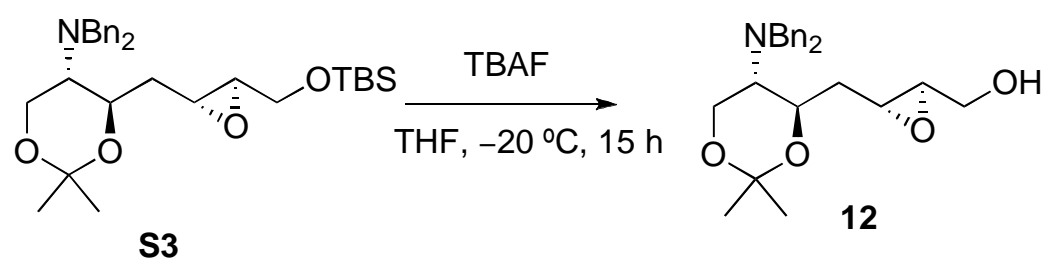

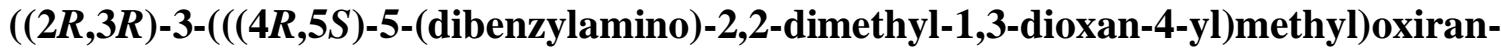
2-yl)methanol (12). Under an atmosphere of nitrogen, TBAF (13 mg, $41 \mu \mathrm{mol})$ was added to a stirred solution of epoxide $\mathbf{S 3}(18 \mathrm{mg}, 35 \mu \mathrm{mol})$ in THF $(400 \mu \mathrm{L})$ at $-20{ }^{\circ} \mathrm{C}$. The mixture was stirred for 15 hours then quenched by addition of water $(5 \mathrm{~mL})$. The mixture was extracted with ethyl acetate $(3 \times 5 \mathrm{~mL})$ and combined extracts washed with brine $(5 \mathrm{~mL})$, dried over $\mathrm{Na}_{2} \mathrm{SO}_{4}$ and concentrated under reduced pressure. Flash chromatography (silica saturated with $\mathrm{Et}_{3} \mathrm{~N}, 1: 3 \mathrm{EtOAc}$ :hexane) provided 12 (11.3 mg, $81 \%$ ) as a viscous oil: IR (neat) $v 3448,2981,2921,1494,1451,1375,1222,1112,746$, $695 \mathrm{~cm}^{-1} ;[\alpha]_{\mathrm{D}}^{23}+15.3^{\circ}\left(\mathrm{CHCl}_{3}, c 1.13\right) ;{ }^{1} \mathrm{H} \mathrm{NMR}\left(400 \mathrm{MHz}, \mathrm{CDCl}_{3}\right) \delta 7.28-7.33(\mathrm{~m}$, $8 \mathrm{H}), 7.22-7.27(\mathrm{~m}, 2 \mathrm{H}), 4.04(\mathrm{td}, J=9.4,2.5 \mathrm{~Hz}, 1 \mathrm{H}), 3.97(\mathrm{dd}, J=12.0,6.4 \mathrm{~Hz}, 1 \mathrm{H})$, $3.91(\mathrm{~d}, J=13.6 \mathrm{~Hz}, 2 \mathrm{H}), 3.89(\mathrm{dd}, J=12.0,5.6 \mathrm{~Hz}, 1 \mathrm{H}), 3.84(\mathrm{ddd}, J=12.4,5.4,3.0$ $\mathrm{Hz}, 1 \mathrm{H}), 3.56(\mathrm{~m}, 1 \mathrm{H}), 3.50(\mathrm{~d}, J=13.6 \mathrm{~Hz}, 2 \mathrm{H}), 3.00(\mathrm{ddd}, J=6.8,4.8,2.0 \mathrm{~Hz}, 1 \mathrm{H})$, $2.84(\mathrm{dt}, J=4.4,2.4 \mathrm{~Hz}, 1 \mathrm{H}), 2.71(\mathrm{dt}, J=9.6,6.2 \mathrm{~Hz}, 1 \mathrm{H}), 2.08$ (ddd, $J=14.4,7.2,2.2$ $\mathrm{Hz}, 1 \mathrm{H}), 1.65$ (t, $J=6.0 \mathrm{~Hz}, 1 \mathrm{H}), 1.49$ (ddd, $J=14.4,9.6,5.0 \mathrm{~Hz}, 1 \mathrm{H}), 1.42(\mathrm{~s}, 3 \mathrm{H})$, $1.31(\mathrm{~s}, 3 \mathrm{H}) ;{ }^{13} \mathrm{C}$ NMR $\left(100 \mathrm{MHz}, \mathrm{CDCl}_{3}\right) \delta 139.5(\mathrm{C}), 129.0(\mathrm{CH}), 128.5(\mathrm{CH}), 127.3$ $(\mathrm{CH}), 99.3(\mathrm{C}), 67.6(\mathrm{CH}), 61.9\left(\mathrm{CH}_{2}\right), 59.0(\mathrm{CH}), 58.0\left(\mathrm{CH}_{2}\right), 57.8(\mathrm{CH}), 54.8\left(\mathrm{CH}_{2}\right)$, 53.2 $(\mathrm{CH}), 35.3\left(\mathrm{CH}_{2}\right), 26.9\left(\mathrm{CH}_{3}\right), 21.6\left(\mathrm{CH}_{3}\right)$; HREIMS $\mathrm{m} / z 397.2250[\mathrm{M}]^{+}$, calcd. for $\mathrm{C}_{24} \mathrm{H}_{31} \mathrm{~N}_{1} \mathrm{O}_{4} 397.2248$.

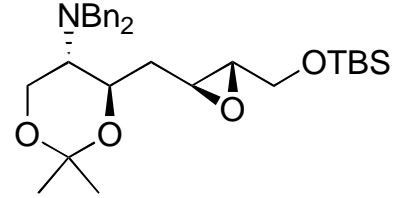

S4

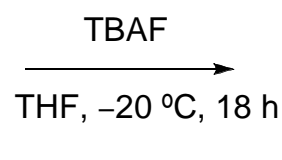
((2S,3S)-3-(((4R,5S)-5-(dibenzylamino)-2,2-dimethyl-1,3-dioxan-4-yl)methyl)oxiran2-yl)methanol (13). Under an atmosphere of nitrogen, TBAF (20 mg, $63 \mu \mathrm{mol})$ was added to a stirred solution of epoxide $\mathbf{S 4}(22 \mathrm{mg}, 43 \mu \mathrm{mol})$ in THF $(400 \mu \mathrm{L})$ at $-20{ }^{\circ} \mathrm{C}$. The mixture was stirred for 18 hours then quenched by addition of water $(2 \mathrm{~mL})$. The mixture was extracted with ethyl acetate $(4 \times 3 \mathrm{~mL})$ and combined extracts washed with brine $(5 \mathrm{~mL})$, dried over $\mathrm{Na}_{2} \mathrm{SO}_{4}$ and concentrated under reduced pressure. Flash chromatography (silica saturated with $\mathrm{Et}_{3} \mathrm{~N}, 1: 3 \mathrm{EtOAc}$ :hexane) provided $\mathbf{1 3}$ (15 mg, $88 \%$ ) as a viscous oil: IR (neat) $v$ 3439, 2989, 2930, 1494, 1460, 1222, 1103, 746, 695 $\mathrm{cm}^{-1} ;[\alpha]_{\mathrm{D}}^{23}+8.1^{\mathrm{o}}\left(\mathrm{CHCl}_{3}, c 1.48\right) ;{ }^{1} \mathrm{H}$ NMR $\left(400 \mathrm{MHz}, \mathrm{CDCl}_{3}\right) \delta 7.29-7.34(\mathrm{~m}, 8 \mathrm{H})$, $7.22-7.28(\mathrm{~m}, 2 \mathrm{H}), 3.80-4.00(\mathrm{~m}, 6 \mathrm{H}), 3.46-3.56(\mathrm{~m}, 3 \mathrm{H}), 2.87-2.94(\mathrm{~m}, 2 \mathrm{H}) 2.77$ (dt, $J=$ 10.0, $6.0 \mathrm{~Hz}, 1 \mathrm{H}$ ), 1.98 (ddd, $J=14.4,6.0,2.8 \mathrm{~Hz}, 1 \mathrm{H}$ ), 1.79 (ddd, $J=14.4,8.0,4.4 \mathrm{~Hz}$, $1 \mathrm{H}), 1.70(\mathrm{t}, J=6.0 \mathrm{~Hz}, 1 \mathrm{H}), 1.39(\mathrm{~s}, 3 \mathrm{H}), 1.30(\mathrm{~s}, 3 \mathrm{H}) ;{ }^{13} \mathrm{C} \mathrm{NMR}\left(100 \mathrm{MHz}, \mathrm{CDCl}_{3}\right) \delta$ $139.5(\mathrm{C}), 128.9(\mathrm{CH}), 128.5(\mathrm{CH}), 127.3(\mathrm{CH}), 99.3(\mathrm{C}), 67.6(\mathrm{CH}), 62.0\left(\mathrm{CH}_{2}\right), 58.0$ 
$\left(\mathrm{CH}_{2}\right), 57.9(\mathrm{CH}), 57.8(\mathrm{CH}), 54.9\left(\mathrm{CH}_{2}\right), 53.4(\mathrm{CH}), 34.3\left(\mathrm{CH}_{2}\right), 26.9\left(\mathrm{CH}_{3}\right), 21.6\left(\mathrm{CH}_{3}\right)$; HREIMS $m / z$ 397.2251 [M] $]^{+}$, calcd. for $\mathrm{C}_{24} \mathrm{H}_{31} \mathrm{~N}_{1} \mathrm{O}_{4} 397.2248$.<smiles>CC1(C)OC[C@@H](S)[C@H](CCC=O)O1</smiles>

12
1) $\mathrm{B}(\mathrm{OMe})_{3}, \mathrm{DMF}, \mathrm{rt}, 30 \mathrm{~min}$

2) $\mathrm{NaN}_{3}, 50 \stackrel{\circ}{\mathrm{C}}, 4 \mathrm{~h}$
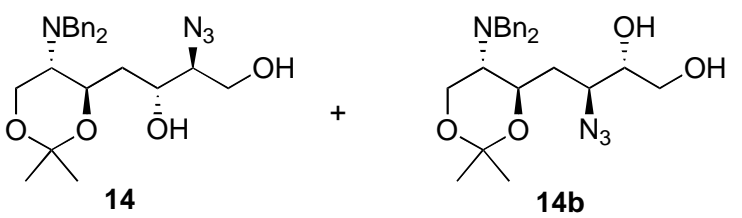

$14: 14 b=9: 1$

(2S,3R)-2-azido-4-((4R,5S)-5-(dibenzylamino)-2,2-dimethyl-1,3-dioxan-4-yl)butane1,3-diol (14). Under an atmosphere of nitrogen, $(\mathrm{MeO})_{3} \mathrm{~B}(6.3 \mu \mathrm{L}, 5.8 \mathrm{mg}, 55 \mu \mathrm{mol})$ was added to a solution of $\mathbf{1 2}(11 \mathrm{mg}, 28 \mu \mathrm{mol})$ in anhydrous DMF $(140 \mu \mathrm{L})$. The solution was stirred for $30 \mathrm{~min}$ at room temperature then $\mathrm{NaN}_{3}(3.6 \mathrm{mg}, 55 \mu \mathrm{mol})$ was added and the reaction was heated to $50^{\circ} \mathrm{C}$ and stirred for 4 hours. The reaction was cooled to room temperature and quenched by addition of a saturated solution of $\mathrm{NaHCO}_{3}(3.0 \mathrm{~mL})$ and the solution stirred a further 30 minutes. The mixture was extracted with ethyl ether $(4 \times$ $3 \mathrm{~mL}$ ) and combined extracts washed with brine $(5 \mathrm{~mL})$, dried over $\mathrm{Na}_{2} \mathrm{SO}_{4}$ and concentrated under reduced pressure. Flash chromatography (silica, 1:3 to 2:3 EtOAc: hexane) provided 14 and $\mathbf{1 4 b}(8.2 \mathrm{mg}$ and $0.8 \mathrm{mg}$ respectively, $74 \%)$ as a viscous oil. Characterization of 14: IR (neat) $v$ 3439, 3032, 2989, 2921, 2887, 2802, 2097, 1494 , $1451,1375,1265,1103,1018,967,823,755,695 \mathrm{~cm}^{-1} ;[\alpha]_{\mathrm{D}}{ }^{24}+10.0^{\circ}\left(\mathrm{CHCl}_{3}, c 0.99\right) ;{ }^{1} \mathrm{H}$ NMR (400 MHz, $\left.\mathrm{CDCl}_{3}\right) \delta 7.25-7.35(\mathrm{~m}, 10 \mathrm{H}), 3.96-4.06(\mathrm{~m}, 3 \mathrm{H}), 4.12-4.20(\mathrm{~m}, 2 \mathrm{H})$, $3.98(\mathrm{dd}, J=12.0,6.4 \mathrm{~Hz}, 1 \mathrm{H}), 3.88-3.98(\mathrm{~m}, 3 \mathrm{H}), 3.80(\mathrm{~m}, 2 \mathrm{H}), 3.68(\mathrm{~m}, 1 \mathrm{H}), 3.52(\mathrm{~d}, J$ $=14.0 \mathrm{~Hz}, 2 \mathrm{H}), 2.28(\mathrm{q}, J=5.4 \mathrm{~Hz}, 1 \mathrm{H}), 2.89(\mathrm{dt}, J=9.6,6.2 \mathrm{~Hz}, 1 \mathrm{H}), 2.50(\mathrm{t}, J=5.6$ $\mathrm{Hz}, 1 \mathrm{H}), 1.94$ (ddd, $J=14.6,8.8,4.4 \mathrm{~Hz}, 1 \mathrm{H}), 1.81$ (ddd, $J=14.6,6.0,2.4 \mathrm{~Hz}, 1 \mathrm{H}), 1.41$ $(\mathrm{s}, 3 \mathrm{H}), 1.31(\mathrm{~s}, 3 \mathrm{H}) ;{ }^{13} \mathrm{C} \mathrm{NMR}\left(100 \mathrm{MHz}, \mathrm{CDCl}_{3}\right) \delta 138.8(\mathrm{C}), 129.2(\mathrm{CH}), 128.6(\mathrm{CH})$, $127.6(\mathrm{CH}), 99.7(\mathrm{C}), 70.2(\mathrm{CH}), 68.7(\mathrm{CH}), 66.3(\mathrm{CH}), 63.2\left(\mathrm{CH}_{2}\right), 57.8\left(\mathrm{CH}_{2}\right), 57.5$ $(\mathrm{CH}), 54.9\left(\mathrm{CH}_{2}\right), 36.0\left(\mathrm{CH}_{2}\right), 26.8\left(\mathrm{CH}_{3}\right), 21.6\left(\mathrm{CH}_{3}\right)$; HREIMS $m / z 440.2417[\mathrm{M}]^{+}$, calcd. for $\mathrm{C}_{24} \mathrm{H}_{32} \mathrm{~N}_{4} \mathrm{O}_{4} 440.2418$.<smiles>CC1(C)OC[C@@H]([NH3+])[C@H](C[C@H](O)[C@H](N)CO)O1</smiles>

14

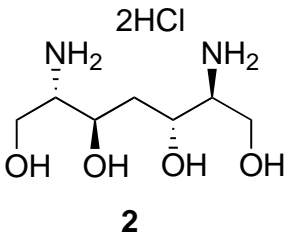

2

(2S,3R,5R,6S)-2,6-diaminoheptane-1,3,5,7-tetraol (2). A mixture of Pd/C (1.5 mg, 1.3 $\mu \mathrm{mol}, 10 \mathrm{~mol} \% \mathrm{Pd})$ and azide $14(6.0 \mathrm{mg}, 13.6 \mu \mathrm{mol})$ in methanol $(0.5 \mathrm{~mL})$ was placed under $\mathrm{H}_{2}(1 \mathrm{~atm})$ and stirred at room temperature. After 16 hours TMSCl $(10.0 \mu \mathrm{L}, 8.5$ $\mathrm{mg}, 80 \mu \mathrm{mol})$ was added and the mixture stirred a further 3 hours. The mixture was filtered through a $0.45 \mu \mathrm{m}$ syringe filter and concentrated under reduced pressure. The crude material was resuspended in water $(0.5 \mathrm{~mL})$ and $\mathrm{Pd} / \mathrm{C}(1.5 \mathrm{mg}, 1.3 \mu \mathrm{mol}, 10 \mathrm{~mol} \%$ $\mathrm{Pd})$ added. The mixture was placed under $\mathrm{H}_{2}(1 \mathrm{~atm})$ and stirred at room temperature for 14 hours. Filtration through a $0.45 \mu \mathrm{m}$ syringe filter and concentration under reduced pressure provided the hydrochloride salt of $2(3.2 \mathrm{mg}, 88 \%)$ as a white solid: ${ }^{1} \mathrm{H}$ NMR 
(400 MHz, $\mathrm{D}_{2} \mathrm{O}$, ref $\left.\mathrm{CH}_{3} \mathrm{CN}\right) \delta 4.16(\mathrm{~m}, 2 \mathrm{H}), 3.93(\mathrm{dd}, J=12.0,4.2 \mathrm{~Hz}, 2 \mathrm{H}), 3.77$ (dd, $J$ $=12.0,8.8 \mathrm{~Hz}, 2 \mathrm{H}), 3.42$ (apparent dt, $J=8.4,4.0 \mathrm{~Hz}, 2 \mathrm{H}), 1.70(\mathrm{dd}, J=8.0,5.2 \mathrm{~Hz}$, $2 \mathrm{H}) ;{ }^{13} \mathrm{C}$ NMR $\left(100 \mathrm{MHz}, \mathrm{D}_{2} \mathrm{O}\right.$, ref $\left.\mathrm{CH}_{3} \mathrm{CN}\right) \delta 65.9(\mathrm{CH}), 58.0\left(\mathrm{CH}_{2}\right), 57.3(\mathrm{CH}), 35.8$ $\left(\mathrm{CH}_{2}\right)$; HREIMS $m / z$ 195.1260 [M] $]^{+}$, calcd. for $\mathrm{C}_{7} \mathrm{H}_{18} \mathrm{~N}_{2} \mathrm{O}_{4}$ 194.1267.

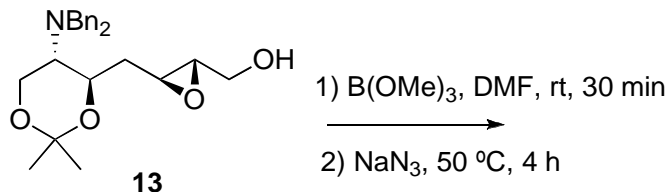

13

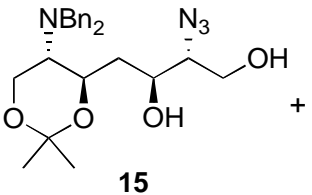

$15: 15 b=3.6: 1$<smiles>CC(C)(C)OC1COC([NH3+])C(CC[C@H](N)[C@H](O)CO)O1</smiles>

$15 b$

(2R,3S)-2-azido-4-((4R,5S)-5-(dibenzylamino)-2,2-dimethyl-1,3-dioxan-4-yl)butane1,3-diol (15). Under an atmosphere of nitrogen, $(\mathrm{MeO})_{3} \mathrm{~B}(8.0 \mu \mathrm{L}, 7.3 \mathrm{mg}, 70 \mu \mathrm{mol})$ was added to a solution of $\mathbf{3}(14 \mathrm{mg}, 35 \mu \mathrm{mol})$ in anhydrous DMF $(180 \mu \mathrm{L})$. The solution was stirred for $30 \mathrm{~min}$ at room temperature then $\mathrm{NaN}_{3}(4.6 \mathrm{mg}, 70 \mu \mathrm{mol})$ was added and the reaction was heated to $50^{\circ} \mathrm{C}$ and stirred for 4 hours. The reaction was cooled to room temperature and quenched by addition of a saturated solution of $\mathrm{NaHCO}_{3}(3.0 \mathrm{~mL})$ and the solution stirred a further 30 minutes. The mixture was extracted with ethyl ether $(4 \times$ $3 \mathrm{~mL}$ ) and combined extracts washed with brine $(5 \mathrm{~mL})$, dried over $\mathrm{Na}_{2} \mathrm{SO}_{4}$ and concentrated under reduced pressure. Flash chromatography (silica, 2:3 EtOAc:hexane) provided 15 and 15b (10.2 $\mathrm{mg}$ and $2.8 \mathrm{mg}$ respectively, $85 \%)$ as viscous oils. Characterization for 15: IR (neat) v 3456, 2989, 2938, 2879, 2089, 1494, 1451, 1383, 1265, 1222, 1069, 967, 891, 823, $738 \mathrm{~cm}^{-1} ;[\alpha]_{\mathrm{D}}{ }^{25}+8.0^{\circ}\left(\mathrm{CHCl}_{3}, c 1.12\right) ;{ }^{1} \mathrm{H} \mathrm{NMR}(400$ $\left.\mathrm{MHz}, \mathrm{CDCl}_{3}\right) \delta 7.24-7.37(\mathrm{~m}, 10 \mathrm{H}), 3.96-4.06(\mathrm{~m}, 3 \mathrm{H}), 3.88-3.94(\mathrm{~m}, 3 \mathrm{H}), 3.74-3.86(\mathrm{~m}$, $2 \mathrm{H}), 3.67(\mathrm{t}, J=7.6 \mathrm{~Hz}, 1 \mathrm{H}), 3.53(\mathrm{~d}, J=13.6 \mathrm{~Hz}, 2 \mathrm{H}), 3.27(\mathrm{dt}, J=6.8,5.2 \mathrm{~Hz}, 1 \mathrm{H})$, $2.78(\mathrm{dt}, J=9.6,6.0 \mathrm{~Hz}, 1 \mathrm{H}), 2.42(\mathrm{t}, J=6.0 \mathrm{~Hz}, 1 \mathrm{H}), 2.32(\mathrm{dt}, J=14.8,2.4 \mathrm{~Hz}, 1 \mathrm{H})$, $1.43(\mathrm{~s}, 3 \mathrm{H}), 1.37(\mathrm{~m}, 1 \mathrm{H}), 1.31(\mathrm{~s}, 3 \mathrm{H}) ;{ }^{13} \mathrm{C} \mathrm{NMR}\left(100 \mathrm{MHz}, \mathrm{CDCl}_{3}\right) \delta 139.1(\mathrm{C}), 129.0$ $(\mathrm{CH}), 128.6(\mathrm{CH}), 127.6(\mathrm{CH}), 99.5(\mathrm{C}), 73.3(\mathrm{CH}), 71.5(\mathrm{CH}), 66.8(\mathrm{CH}), 63.2\left(\mathrm{CH}_{2}\right)$, $58.4(\mathrm{CH}), 57.8\left(\mathrm{CH}_{2}\right), 55.2\left(\mathrm{CH}_{2}\right), 36.7\left(\mathrm{CH}_{2}\right), 26.9\left(\mathrm{CH}_{3}\right), 21.8\left(\mathrm{CH}_{3}\right) ; \mathrm{HREIMS} \mathrm{m} / \mathrm{z}$ $440.2429[\mathrm{M}]^{+}$, calcd. for $\mathrm{C}_{24} \mathrm{H}_{32} \mathrm{~N}_{4} \mathrm{O}_{4} 440.2418$.

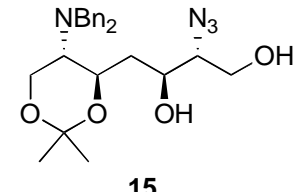

15

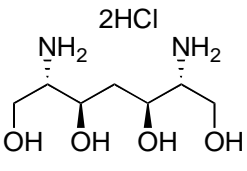

3

(2R,3S,5R,6S)-2,6-diaminoheptane-1,3,5,7-tetraol (3). A mixture of $\mathrm{Pd} / \mathrm{C}$ (1.5 mg, 1.3 $\mu \mathrm{mol}, 10 \mathrm{~mol} \% \mathrm{Pd})$ and azide $15(6.0 \mathrm{mg}, 13.6 \mu \mathrm{mol})$ in methanol $(0.5 \mathrm{~mL})$ was placed under $\mathrm{H}_{2}(1 \mathrm{~atm})$ and stirred at room temperature. After 16 hours TMSCl $(10.0 \mu \mathrm{L}, 8.5$ $\mathrm{mg}, 80 \mu \mathrm{mol})$ was added and the mixture stirred a further 3 hours. The mixture was filtered through a $0.45 \mu \mathrm{m}$ syringe filter and concentrated under reduced pressure. The crude material was resuspended in water $(0.5 \mathrm{~mL})$ and $\mathrm{Pd} / \mathrm{C}(1.5 \mathrm{mg}, 1.3 \mu \mathrm{mol}, 10 \mathrm{~mol} \%$ $\mathrm{Pd})$ added. The mixture was placed under $\mathrm{H}_{2}(1 \mathrm{~atm})$ and stirred at room temperature for 14 hours. Filtration through a $0.45 \mu \mathrm{m}$ syringe filter and concentration under reduced pressure provided the hydrochloride salt of $\mathbf{3}(2.5 \mathrm{mg}, 69 \%)$ as a white solid: ${ }^{1} \mathrm{H}$ NMR 
(400 MHz, $\mathrm{D}_{2} \mathrm{O}$, ref $\mathrm{CH}_{3} \mathrm{CN}$ ) $\delta 4.16$ (apparent $\mathrm{p}, J=4.4 \mathrm{~Hz}, 2 \mathrm{H}$ ), 3.93 (dd, $J=12.0,4.4$ $\mathrm{Hz}, 2 \mathrm{H}), 3.78$ (dd, $J=12.0,8.2 \mathrm{~Hz}, 2 \mathrm{H}), 3.45$ (apparent p, $J=4.0 \mathrm{~Hz}, 2 \mathrm{H}), 1.88$ (dt, $J=$ $10.3,4.4 \mathrm{~Hz}, 1 \mathrm{H}), 1.82(\mathrm{dt}, J=10.3,8.4 \mathrm{~Hz}, 1 \mathrm{H}) ;{ }^{13} \mathrm{C} \mathrm{NMR}\left(100 \mathrm{MHz}, \mathrm{D}_{2} \mathrm{O}\right.$, ref $\left.\mathrm{CH}_{3} \mathrm{CN}\right)$ $\delta 67.4(\mathrm{CH}), 58.0\left(\mathrm{CH}_{2}\right), 56.6(\mathrm{CH}), 35.3\left(\mathrm{CH}_{2}\right)$; HRESIMS $m / z 195.1333[\mathrm{M}+\mathrm{H}]^{+}$, calcd. for $\mathrm{C}_{7} \mathrm{H}_{19} \mathrm{~N}_{2} \mathrm{O}_{4} 195.1339$.

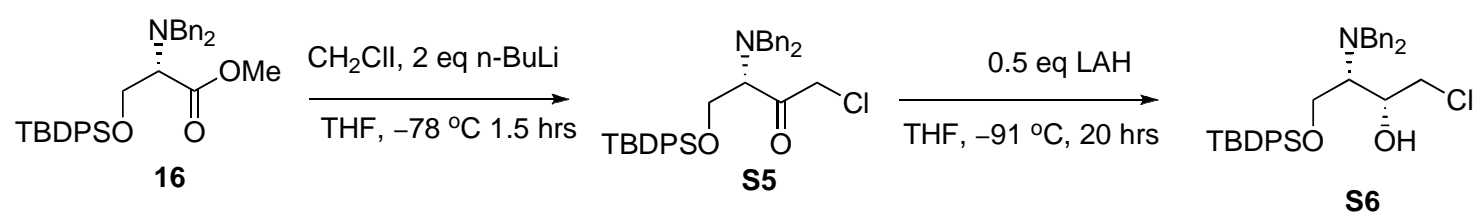

(2R,3S)-4-(tert-butyldiphenylsilyloxy)-1-chloro-3-(dibenzylamino)butan-2-ol (S5).

Under an atmosphere of nitrogen, $n$-BuLi $(7.50 \mathrm{~mL}, 1.87 \mathrm{mmol}, 2.5 \mathrm{M}$ in hexane) was added dropwise to a stirred solution of ester $16(5.04 \mathrm{~g}, 0.94 \mathrm{mmol})$ and chloroiodomethane $(1.36 \mathrm{~mL}, 1.87 \mathrm{mmol})$ in anhydrous $\mathrm{THF}$ at $-78{ }^{\circ} \mathrm{C}$. The mixture was stirred for 90 min then quenched with saturated aqueous $\mathrm{NH}_{4} \mathrm{Cl}(50 \mathrm{~mL})$. The mixture was extracted with dichloromethane $(4 \times 25 \mathrm{~mL})$ and combined extracts washed with brine $(50 \mathrm{~mL})$, dried over $\mathrm{Na}_{2} \mathrm{SO}_{4}$ and concentrated under reduced pressure, providing crude $\mathbf{S 5}$ $(5.7 \mathrm{~g})$ as a yellow viscous oil. The crude ketone $\mathbf{S 5}$ was reduced without further purification. Under an atmosphere of nitrogen, LAH (0.47 mL, $1 \mathrm{M}$ in THF) was added dropwise to a stirred solution of ketone S5 $(5.2 \mathrm{~g}, 0.93 \mathrm{mmol})$ in anhydrous THF (45 $\mathrm{mL})$ at $-91{ }^{\circ} \mathrm{C}$. The mixture was stirred for 20 hours then quenched by addition of dropwise addition of water $(5 \mathrm{~mL})$. The solution was stirred at $-91{ }^{\circ} \mathrm{C}$ for 1 hour then quenched with $30 \mathrm{~mL}$ saturated aqueous $\mathrm{NH}_{4} \mathrm{Cl}$. The mixture was extracted with dichloromethane $(3 \times 50 \mathrm{~mL})$ and combined extracts washed with brine $(50 \mathrm{~mL})$, dried over $\mathrm{Na}_{2} \mathrm{SO}_{4}$ and concentrated under reduced pressure. Recrystallization from 30:1 hexane : dichloromethane gave pure $\mathbf{S 6}$ (3.82 g) as a white crystals. The mother liquor was concentrated under reduced pressure and chromatographed on silica (1:19 EtOAc:hexane) providing additional $\mathbf{S 6}(336 \mathrm{mg})$ as a mixture with other diastereomers. Combined yield was $80 \%$ over two steps, de $=94 \%$ based on NMR: IR (neat) $v 3415$, 3065, 3030, 2925, 2855, 1955, 1885, 1816, 1588, 1495, 1472, 1452, 1425, 1390, 1359, 1262, 1105, 742, 703, 610, $501 \mathrm{~cm}^{-1} ;[\alpha]_{\mathrm{D}}{ }^{24} 41.4^{\mathrm{o}}\left(\mathrm{CHCl}_{3}, c 14.1\right) ;{ }^{1} \mathrm{H} \mathrm{NMR}(400 \mathrm{MHz}$, $\left.\mathrm{CDCl}_{3}\right) \delta 7.70(\mathrm{~d}, J=8.0 \mathrm{~Hz}, 4 \mathrm{H}), 7.40-7.55(\mathrm{~m}, 6 \mathrm{H}), 7.20-7.38(\mathrm{~m}, 10 \mathrm{H}), 4.31(\mathrm{~s}, 1 \mathrm{H})$, $3.96(\mathrm{~d}, J=13.2 \mathrm{~Hz}, 2 \mathrm{H}), 3.91(\mathrm{bm}, 3 \mathrm{H}), 3.59(\mathrm{dd}, J=11.6,2.6 \mathrm{~Hz}, 1 \mathrm{H}), 3.54(\mathrm{~d}, J=$ $13.2 \mathrm{~Hz}, 2 \mathrm{H}), 3.34(\mathrm{dd}, J=11.6,5.6 \mathrm{~Hz}, 1 \mathrm{H}), 2.92(\mathrm{dt}, J=8.8,5.2 \mathrm{~Hz}, 1 \mathrm{H}), 1.11(\mathrm{~s}, 9 \mathrm{H})$; ${ }^{13} \mathrm{C} \mathrm{NMR}\left(100 \mathrm{MHz}, \mathrm{CDCl}_{3}\right) \delta 138.7(\mathrm{C}), 135.7(\mathrm{CH}), 135.6(\mathrm{CH}), 132.7(\mathrm{C}), 132.6(\mathrm{C})$, $130.1(\mathrm{CH}), 130.0(\mathrm{CH}), 129.0(\mathrm{CH}), 128.5(\mathrm{CH}), 127.9(\mathrm{CH}), 127.3(\mathrm{CH}), 68.3(\mathrm{CH})$, $61.1(\mathrm{CH}), 60.3\left(\mathrm{CH}_{2}\right), 54.8\left(\mathrm{CH}_{2}\right), 47.8\left(\mathrm{CH}_{2}\right), 27.1\left(\mathrm{CH}_{3}\right), 19.4(\mathrm{C}) ; \mathrm{HRMS} \mathrm{m} / z$ 557.2520 [M] $]^{+}$, calcd. for $\mathrm{C}_{34} \mathrm{H}_{40} \mathrm{Cl}_{1} \mathrm{~N}_{1} \mathrm{O}_{2} \mathrm{Si}_{1}$ 557.2517. 


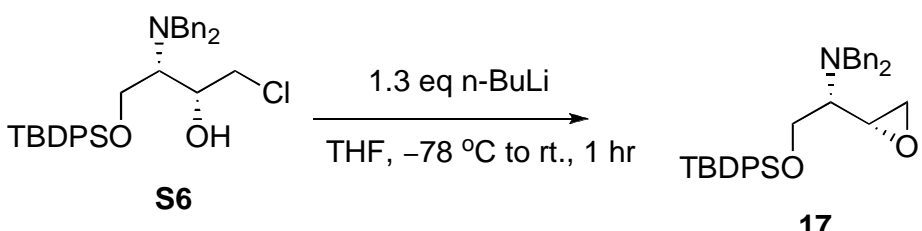

17

(S)-N,N-dibenzyl-2-(tert-butyldiphenylsilyloxy)-1-((R)-oxiran-2-yl)ethanamine (17). Under an atmosphere of nitrogen, $n$-BuLi $(197 \mu \mathrm{L}, 492 \mu \mathrm{mol}, 2.5 \mathrm{M}$ in hexane) was added dropwise to a stirred solution of alcohol $\mathbf{S 6}(211 \mathrm{mg}, 379 \mu \mathrm{mol})$ in anhydrous THF at $-78{ }^{\circ} \mathrm{C}$. The stirred mixture was warmed to room temperature for 45 min then quenched with saturated aqueous $\mathrm{NH}_{4} \mathrm{Cl}(10 \mathrm{~mL})$. The mixture was extracted with dichloromethane $(3 \times 15 \mathrm{~mL})$ and combined extracts washed with brine $(20 \mathrm{~mL})$, dried over $\mathrm{Na}_{2} \mathrm{SO}_{4}$ and concentrated under reduced pressure. Flash chromatography on triethylamine saturated silica (3\% ethyl acetate in hexane) provided $17(181 \mathrm{mg}, 91 \%)$ as a light yellow viscous oil: IR (neat) v 3458, 3065, 3030, 2960, 2917, 2855, 1947, 1894, $1816,1588,1495,1472,1452,1425,1359,1254,1115,823,742,695,610 \mathrm{~cm}^{-1} ;[\alpha]_{\mathrm{D}}{ }^{24}$ 21.0 $0^{\circ}\left(\mathrm{CHCl}_{3}, c\right.$ 8.91); ${ }^{1} \mathrm{H}$ NMR $\left(400 \mathrm{MHz}, \mathrm{CDCl}_{3}\right) \delta 7.64-7.72(\mathrm{~m}, 4 \mathrm{H}), 7.38-7.52(\mathrm{~m}$, $10 \mathrm{H}), 7.30-7.36(\mathrm{~m}, 4 \mathrm{H}), 7.22-7.28(\mathrm{~m}, 2 \mathrm{H}) 3.84-4.00(\mathrm{~m}, 6 \mathrm{H}), 3.26(\mathrm{ddd}, J=4.8,4.4$, $2.4 \mathrm{~Hz}, 1 \mathrm{H}), 2.77(\mathrm{dd}, J=4.8,4.4 \mathrm{~Hz}, 1 \mathrm{H}), 2.72(\mathrm{q}, J=6.4 \mathrm{~Hz}, 1 \mathrm{H}), 2.60(\mathrm{dd}, J=4.8,2.4$ $\mathrm{Hz}, 1 \mathrm{H}), 1.10(\mathrm{~s}, 9 \mathrm{H}) ;{ }^{13} \mathrm{C} \mathrm{NMR}\left(100 \mathrm{MHz}, \mathrm{CDCl}_{3}\right) \delta 140.2(\mathrm{C}), 135.52(\mathrm{CH}), 135.49$ $(\mathrm{CH}), 133.2(\mathrm{C}), 133.1(\mathrm{C}), 129.7(\mathrm{CH}), 128.6(\mathrm{CH}), 128.1(\mathrm{CH}), 127.7(\mathrm{CH}), 126.7$ $(\mathrm{CH}), 63.5\left(\mathrm{CH}_{2}\right), 61.4(\mathrm{CH}), 55.6\left(\mathrm{CH}_{2}\right), 51.8(\mathrm{CH}), 44.9\left(\mathrm{CH}_{2}\right), 27.0\left(\mathrm{CH}_{3}\right), 19.3(\mathrm{C})$; HRMS $m / z$, $521.2752[\mathrm{M}]^{+}$, calcd. for $\mathrm{C}_{34} \mathrm{H}_{39} \mathrm{~N}_{1} \mathrm{O}_{2} \mathrm{Si}_{1} 521.2750$.
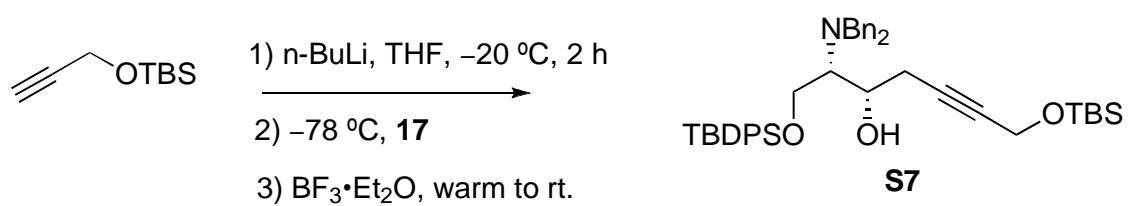

(6S,7S)-6-(dibenzylamino)-2,2,13,13,14,14-hexamethyl-3,3-diphenyl-4,12-dioxa-3,13disilapentadec-9-yn-7-ol (S7). Under an atmosphere of nitrogen, n-BuLi (2.1 mL, 5.25 mmol, $2.5 \mathrm{M}$ in hexane) was added dropwise to a stirred solution of $O-t$ butyldimethysilyl propargyl ether $(970 \mathrm{mg}, 5.73 \mathrm{mmol})$ in anhydrous THF $(16 \mathrm{~mL})$ at $20^{\circ} \mathrm{C}$. The mixture was stirred for 1 hour then cooled to $-78^{\circ} \mathrm{C}$ and epoxide $17(2.49 \mathrm{~g}$, $4.77 \mathrm{mmol}$ in THF $(8 \mathrm{~mL})$ ) was added dropwise followed by slow addition of $\mathrm{BF}_{3} \cdot \mathrm{Et}_{2} \mathrm{O}$ (605 $\mu \mathrm{L}, 4.77 \mathrm{mmol})$. The mixture was stirred for 1 hour then warmed to room temperature overnight. The solution was cooled to $-78^{\circ} \mathrm{C}$ and quenched with $25 \mathrm{~mL}$ saturated aqueous $\mathrm{NH}_{4} \mathrm{Cl}$, extracted with dichloromethane $(3 \times 50 \mathrm{~mL})$ and combined extracts washed with brine $(50 \mathrm{~mL})$, dried over $\mathrm{Na}_{2} \mathrm{SO}_{4}$ and concentrated under reduced pressure. Flash chromatography (silica, 7\% ethyl acetate in hexane) provided S7 (2.63 g, $80 \%$ ) as a viscous oil: IR (neat) $v 3439,3067,2960,2919,2853,1475,1425,1244,1079$, $831,691 \mathrm{~cm}^{-1} ;[\alpha]_{\mathrm{D}}{ }^{24}+28.1^{\circ}\left(\mathrm{CHCl}_{3}, c 7.54\right) ;{ }^{1} \mathrm{H} \mathrm{NMR}\left(400 \mathrm{MHz}, \mathrm{CDCl}_{3}\right) \delta 7.71(\mathrm{bs}$, $4 \mathrm{H}), 7.40-7.52(\mathrm{~m}, 6 \mathrm{H}), 7.20-7.30(\mathrm{~m}, 10 \mathrm{H}), 4.29$ (bs, 1H), 4.11 (dt, $J=15.6,2.0 \mathrm{~Hz}$, $1 \mathrm{H}), 4.06(\mathrm{dt}, J=15.6,2.0 \mathrm{~Hz}, 1 \mathrm{H}), 3.97(\mathrm{~m}, 3 \mathrm{H}), 3.76-3.90(\mathrm{~m}, 2 \mathrm{H}), 3.58(\mathrm{~d}, J=13.2$ $\mathrm{Hz}, 2 \mathrm{H}), 2.88$ (bs, 1H), 2.43 (bd, $J=17.6 \mathrm{~Hz}, 1 \mathrm{H}), 2.17$ (bd, $J=17.6 \mathrm{~Hz}, 1 \mathrm{H}), 1.12$ (s, 
9H), $0.88(\mathrm{~s}, 9 \mathrm{H}), 0.06(\mathrm{~s}, 6 \mathrm{H}) ;{ }^{13} \mathrm{C} \mathrm{NMR}\left(100 \mathrm{MHz}, \mathrm{CDCl}_{3}\right) \delta 138.9(\mathrm{C}), 135.7(\mathrm{CH})$, $135.6(\mathrm{CH}), 132.8(\mathrm{C}), 132.7(\mathrm{C}), 130.0(\mathrm{CH}), 129.9(\mathrm{CH}), 129.1(\mathrm{CH}), 128.4(\mathrm{CH})$, $127.9(\mathrm{CH}), 127.2(\mathrm{CH}), 81.4(\mathrm{C}), 80.5(\mathrm{C}), 65.9(\mathrm{CH}), 62.4\left(\mathrm{CH}_{2}\right), 60.3(\mathrm{CH}), 54.7$ $\left(\mathrm{CH}_{2}\right), 51.9\left(\mathrm{CH}_{2}\right), 27.2\left(\mathrm{CH}_{3}\right), 26.1\left(\mathrm{CH}_{3}\right), 24.5\left(\mathrm{CH}_{2}\right), 19.4(\mathrm{C}), 18.5(\mathrm{C}),-4.80\left(\mathrm{CH}_{3}\right),-$ $4.84\left(\mathrm{CH}_{3}\right)$; HREIMS m/z $691.3875[\mathrm{M}]^{+}$, calcd. for $\mathrm{C}_{43} \mathrm{H}_{57} \mathrm{~N}_{1} \mathrm{O}_{3} \mathrm{Si}_{2} 691.3871$.

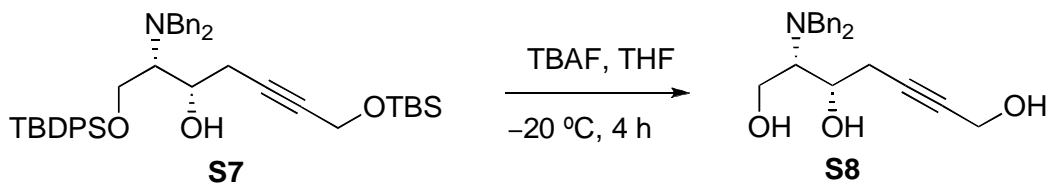

(2S,3S)-2-(dibenzylamino)hept-5-yne-1,3,7-triol (S8). Under an atmosphere of nitrogen TBAF (2.60 g, $8.24 \mathrm{mmol})$ was added to a stirred solution of alkyne $\mathbf{S 7}$ (2.48 g, 3.58 $\mathrm{mmol})$ in THF $(20 \mathrm{~mL})$ at $-20^{\circ} \mathrm{C}$. The mixture was stirred for 4 hours then quenched by addition of water $(75 \mathrm{~mL})$. The mixture was extracted with ethyl acetate $(4 \times 50 \mathrm{~mL})$ and combined extracts washed with brine $(100 \mathrm{~mL})$, dried over $\mathrm{Na}_{2} \mathrm{SO}_{4}$ and concentrated under reduced pressure. Flash chromatography (silica, 1:1 EtOAc:hexane then 6:94 MeOH: $\left.\mathrm{CH}_{2} \mathrm{Cl}_{2}\right)$ provided S8 $(0.99 \mathrm{~g}, 82 \%)$ as a viscous oil: IR (neat) $v$ 3373, 2927 , $1861,1491,1458,1136,1070,1013,763,695 \mathrm{~cm}^{-1} ;[\alpha]_{\mathrm{D}}{ }^{25}+31.5^{\circ}\left(\mathrm{CHCl}_{3}, c 9.05\right) ;{ }^{1} \mathrm{H}$ NMR (400 MHz, $\left.\mathrm{CDCl}_{3}\right) \delta 7.22-7.35(\mathrm{~m}, 10 \mathrm{H}), 4.09(\mathrm{~b}, 2 \mathrm{H}), 3.98(\mathrm{~d}, J=13.2 \mathrm{~Hz}, 2 \mathrm{H})$, $3.80-3.88(\mathrm{~m}, 3 \mathrm{H}), 3.68(\mathrm{~d}, J=13.2 \mathrm{~Hz}, 2 \mathrm{H}), 2.88(\mathrm{dt}, J=9.2,6.4 \mathrm{~Hz}, 1 \mathrm{H}), 2.49(\mathrm{dm}, J=$ $17.2 \mathrm{~Hz}, 1 \mathrm{H}), 2.37(\mathrm{dm}, J=17.2 \mathrm{~Hz}, 1 \mathrm{H}) ;{ }^{13} \mathrm{C} \mathrm{NMR}\left(100 \mathrm{MHz}, \mathrm{CDCl}_{3}\right) \delta 139.0(\mathrm{C})$, $129.3(\mathrm{CH}), 128.6(\mathrm{CH}), 127.4(\mathrm{CH}), 82.2(\mathrm{C}), 80.8(\mathrm{C}), 67.1(\mathrm{CH}), 62.2(\mathrm{CH}), 58.5$ $\left(\mathrm{CH}_{2}\right), 54.6\left(\mathrm{CH}_{2}\right), 50.8\left(\mathrm{CH}_{2}\right), 24.5\left(\mathrm{CH}_{2}\right)$; HREIMS $\mathrm{m} / z 339.1835[\mathrm{M}]^{+}$, calcd. for $\mathrm{C}_{21} \mathrm{H}_{25} \mathrm{~N}_{1} \mathrm{O}_{3} 339.1829$.
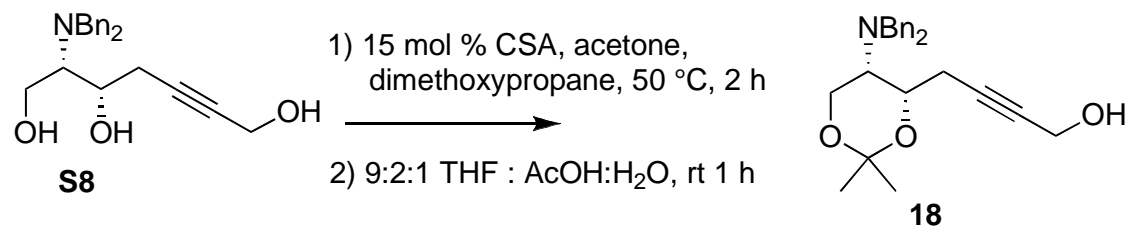

4-((4S,5S)-5-(dibenzylamino)-2,2-dimethyl-1,3-dioxan-4-yl)but-2-yn-1-ol (18). A sealed vial containing alkyne S8 $(842 \mathrm{mg}, 2.48 \mathrm{mmol}$, in 1:1 2,2-dimethoxypropane /acetone $(10 \mathrm{~mL}))$ and CSA $(120 \mathrm{mg}, 520 \mu \mathrm{mol})$ was heated at $50{ }^{\circ} \mathrm{C}$ with stirring for 14 hours. The stirred mixture was cooled to room temperature and quenched with saturated aqueous $\mathrm{NaHCO}_{3}(50 \mathrm{~mL})$. The mixture was extracted with ethyl ether $(3 \times 50 \mathrm{~mL})$ and combined extracts washed with brine $(100 \mathrm{~mL})$, dried over $\mathrm{Na}_{2} \mathrm{SO}_{4}$ and concentrated under reduced pressure. The crude product was redissolved in $4 \mathrm{~mL}$ of 4:2:1 THF/acetic acid/water and stirred for 1 hour at room temperature. The stirred mixture was quenched with saturated aqueous $\mathrm{NaHCO}_{3}(100 \mathrm{~mL})$ extracted with ethyl ether $(3 \times 50 \mathrm{~mL})$ and combined extracts washed with brine $(50 \mathrm{~mL})$, dried over $\mathrm{Na}_{2} \mathrm{SO}_{4}$ and concentrated under reduced pressure. Flash chromatography (Analogix $12 \mathrm{~g}$ silica cartridge, 1:3 EtOAc:hexane, $20 \mathrm{~mL} / \mathrm{min}$ flow rate) provided $18(755 \mathrm{mg}, 80 \%)$ as a viscous oil: IR (neat) v 3439, 3032, 2989, 2930, 2862, 2802, 1604, 1494, 1451, 1383, 1188, 1137, 1103, $1010,746,695 \mathrm{~cm}^{-1} ;[\alpha]_{\mathrm{D}}{ }^{24}+97.1^{\circ}\left(\mathrm{CHCl}_{3}, c 6.58\right) ;{ }^{1} \mathrm{H} \mathrm{NMR}\left(400 \mathrm{MHz}, \mathrm{CDCl}_{3}\right) \delta 7.43$ 
(bd, $J=7.2 \mathrm{~Hz}, 4 \mathrm{H}), 7.35$ (bt, $J=7.2 \mathrm{~Hz}, 4 \mathrm{H}), 7.26$ (bt, $J=7.2 \mathrm{~Hz}, 2 \mathrm{H}), 4.43(\mathrm{~d}, J=12.8$ $\mathrm{Hz}, 1 \mathrm{H}), 4.33$ (bd, $J=14.0 \mathrm{~Hz}, 2 \mathrm{H}), 4.17$ (m, 1H), 4.11 (bs, 2H), 3.97 (dd, $J=12.8,3.2$ $\mathrm{Hz}, 1 \mathrm{H}), 2.59(\mathrm{~d}, J=14.0 \mathrm{~Hz}, 2 \mathrm{H}), 2.90(\mathrm{ddt}, J=18.0,7.6,2.0 \mathrm{~Hz}, 1 \mathrm{H}), 2.64$ (ddt, $J=$ 18.0, 6.0, 2.0 Hz, 1H), $2.53(\mathrm{t}, J=3.4 \mathrm{~Hz}, 1 \mathrm{H}), 1.49(\mathrm{~s}, 3 \mathrm{H}), 1.44(\mathrm{~s}, 3 \mathrm{H}) ;{ }^{13} \mathrm{C} \mathrm{NMR}(100$ $\left.\mathrm{MHz}, \mathrm{CDCl}_{3}\right) \delta 140.2(\mathrm{C}), 128.9(\mathrm{CH}), 128.3(\mathrm{CH}), 126.9(\mathrm{CH}), 99.0(\mathrm{C}), 83.0(\mathrm{C}), 79.7$ (C), $72.1(\mathrm{CH}), 58.4\left(\mathrm{CH}_{2}\right), 56.1\left(\mathrm{CH}_{2}\right), 51.2\left(\mathrm{CH}_{2}\right), 50.2(\mathrm{CH}), 29.5\left(\mathrm{CH}_{3}\right), 22.3\left(\mathrm{CH}_{2}\right)$, $18.7\left(\mathrm{CH}_{3}\right)$; HREIMS $m / z$ 379.2136 [M] ${ }^{+}$, calcd. for $\mathrm{C}_{24} \mathrm{H}_{29} \mathrm{~N}_{1} \mathrm{O}_{3} 379.2142$.
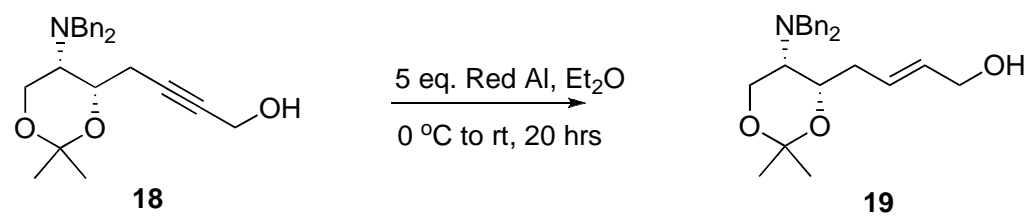

(E)-4-((4S,5S)-5-(dibenzylamino)-2,2-dimethyl-1,3-dioxan-4-yl)but-2-en-1-ol (19). Under an atmosphere of nitrogen, Red-Al $65 \mathrm{wt} \%$ in toluene $(764 \mu \mathrm{L}, 2.67 \mathrm{mmol})$ was added dropwise to a stirred solution of alkyne $18(191 \mathrm{mg}, 535 \mu \mathrm{mol})$ in anhydrous ethyl ether $(5.0 \mathrm{~mL})$ at $-10^{\circ} \mathrm{C}$. The mixture was allowed to warm to room temperature and stirred overnight. After 20 hours the reaction was cooled to $-10^{\circ} \mathrm{C}$ and quenched by dropwise addition of a 1:3 $\mathrm{H}_{2} 0$ :THF $(1.5 \mathrm{~mL})$, warmed to room temperature and added to saturated aqueous $\mathrm{NH}_{4} \mathrm{Cl}(5 \mathrm{~mL})$. The mixture was extracted with ethyl ether $(4 \times 5 \mathrm{~mL})$ and combined extracts washed with water $(5 \mathrm{~mL})$, brine $(5 \mathrm{~mL})$, dried over $\mathrm{Na}_{2} \mathrm{SO}_{4}$ and concentrated under reduced pressure. Flash chromatography (Analogix $4 \mathrm{~g}$ silica cartridge, $20 \%$ ethyl acetate in hexane, $13 \mathrm{~mL} / \mathrm{min}$ flow rate) provided $19(183.1 \mathrm{mg}$, $95 \%$ ) as a viscous oil: IR (neat) $v$ 3406, 3026, 2993, 2935, 2861, 2795, 2366, 2325, 1491, 1458, 1376, 1367, 1260, 1194, 1095, 1004, 963, 740, $699 \mathrm{~cm}^{-1} ;[\alpha]_{\mathrm{D}}^{23}+42.8^{\circ}\left(\mathrm{CHCl}_{3}, c\right.$ 8.39); ${ }^{1} \mathrm{H} \mathrm{NMR}\left(400 \mathrm{MHz}, \mathrm{CDCl}_{3}\right) \delta 7.44(\mathrm{~d}, J=7.2 \mathrm{~Hz}, 4 \mathrm{H}), 7.36(\mathrm{t}, J=7.2 \mathrm{~Hz}, 4 \mathrm{H})$, $7.27(\mathrm{t}, J=7.2 \mathrm{~Hz}, 2 \mathrm{H}), 5.72(\mathrm{dt}, J=15.2,6.0 \mathrm{~Hz}, 1 \mathrm{H}), 5.55(\mathrm{dt}, J=15.2,7.2 \mathrm{~Hz}, 1 \mathrm{H})$, $4.45(\mathrm{~d}, J=12.8 \mathrm{~Hz}, 1 \mathrm{H}), 4.37(\mathrm{bd}, J=14.0 \mathrm{~Hz}, 2 \mathrm{H}), 4.02-3.97(\mathrm{~m}, 3 \mathrm{H}), 3.95(\mathrm{dd}, J=$ $12.8,3.6 \mathrm{~Hz}, 1 \mathrm{H}), 3.59(\mathrm{~d}, J=14.0 \mathrm{~Hz}, 2 \mathrm{H}), 2.59(\mathrm{t}, J=6.4 \mathrm{~Hz}, 2 \mathrm{H}), 2.37(\mathrm{t}, J=3.2 \mathrm{~Hz}$, 1H), 2.03 (bs, 1H), 1.47 (s, 3H), $1.45(\mathrm{~s}, 3 \mathrm{H}) ;{ }^{13} \mathrm{C} \mathrm{NMR}\left(100 \mathrm{MHz}, \mathrm{CDCl}_{3}\right) \delta 140.3(\mathrm{C})$, $131.2(\mathrm{CH}), 129.0(\mathrm{CH}), 128.8(\mathrm{CH}), 128.2(\mathrm{CH}), 126.8(\mathrm{CH}), 98.6(\mathrm{C}), 72.7(\mathrm{CH}), 63.4$ $\left(\mathrm{CH}_{2}\right), 58.1\left(\mathrm{CH}_{2}\right), 56.0\left(\mathrm{CH}_{2}\right), 50.6(\mathrm{CH}), 34.6\left(\mathrm{CH}_{2}\right), 29.6\left(\mathrm{CH}_{3}\right), 18.7\left(\mathrm{CH}_{3}\right) ; \mathrm{HRMS}$ $m / z 381.2302[\mathrm{M}]^{+}$, calcd. for $\mathrm{C}_{24} \mathrm{H}_{31} \mathrm{~N}_{1} \mathrm{O}_{3} 381.2298$.

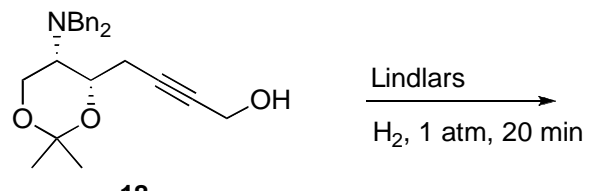

18

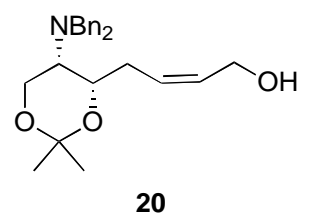

20

(Z)-4-((4S,5S)-5-(dibenzylamino)-2,2-dimethyl-1,3-dioxan-4-yl)but-2-en-1-ol (20). To a solution of alkyne $18(25 \mathrm{mg}, 66 \mu \mathrm{mol})$ in 1:1 ethanol:hexane ethyl ether $(5.0 \mathrm{~mL})$ was added quinoline $(100 \mu \mathrm{L}$ of $20 \mu \mathrm{L} / 10 \mathrm{~mL}$ solution in hexane) and Lindlar catalyst (14 $\mathrm{mg}, 6.6 \mu \mathrm{mol})$. The mixture was placed under hydrogen (1 atm) at room temperature and stirred for 20 minutes. The solution was filtered through a $0.45 \mu \mathrm{m}$ syringe filter and 
concentrated under reduced pressure to provided $18(25 \mathrm{mg}, 99 \%)$ as a viscous oil: IR (neat) v 3423, 2026, 2992, 2923, 2854, 1493, 1450, 1381, 1260, 1200, 1148, 1070, 1010, $958,898,821,752,700 \mathrm{~cm}^{-1} ;[\alpha]_{\mathrm{D}}{ }^{22}+9.6^{\circ}\left(\mathrm{CHCl}_{3}, c 2.44\right) ;{ }^{1} \mathrm{H} \mathrm{NMR}\left(400 \mathrm{MHz}, \mathrm{CDCl}_{3}\right)$ $\delta 7.41(\mathrm{~d}, J=7.0 \mathrm{~Hz}, 4 \mathrm{H}), 7.33(\mathrm{t}, J=7.0 \mathrm{~Hz}, 4 \mathrm{H}), 7.24(\mathrm{t}, J=7.0 \mathrm{~Hz}, 2 \mathrm{H}), 5.81(\mathrm{dt}, J=$ $10.8,7.2 \mathrm{~Hz}, 1 \mathrm{H}), 5.58-5.50(\mathrm{~m}, 1 \mathrm{H}), 4.38(\mathrm{~d}, J=13.2 \mathrm{~Hz}, 1 \mathrm{H}), 4.32$ (bd, $J=14.0 \mathrm{~Hz}$, 2H), $4.21(\mathrm{dd}, J=11.6,7.6 \mathrm{~Hz}, 1 \mathrm{H}), 4.02-3.90(\mathrm{~m}, 3 \mathrm{H}), 3.56(\mathrm{~d}, J=14.0 \mathrm{~Hz}, 2 \mathrm{H}), 2.92$ $(\mathrm{dtd}, J=14.8,9.6,0.8 \mathrm{~Hz}, 1 \mathrm{H}), 2.36(\mathrm{t}, J=3.2 \mathrm{~Hz}, 1 \mathrm{H}), 2.24(\mathrm{~m}, 1 \mathrm{H}), 1.42(\mathrm{~s}, 3 \mathrm{H}), 1.40$ $(\mathrm{s}, 3 \mathrm{H}) ;{ }^{13} \mathrm{C} \mathrm{NMR}\left(100 \mathrm{MHz}, \mathrm{CDCl}_{3}\right) \delta 140.4(\mathrm{C}), 131.0(\mathrm{CH}), 130.1(\mathrm{CH}), 128.8(\mathrm{CH})$, $128.4(\mathrm{CH}), 127.0(\mathrm{CH}), 99.1(\mathrm{C}), 72.0(\mathrm{CH}), 58.3\left(\mathrm{CH}_{2}\right), 57.7\left(\mathrm{CH}_{2}\right), 56.1\left(\mathrm{CH}_{2}\right), 51.8$ $(\mathrm{CH}), 30.5\left(\mathrm{CH}_{2}\right), 29.4\left(\mathrm{CH}_{3}\right), 18.9\left(\mathrm{CH}_{3}\right)$; HRMS $m / z 382.2380[\mathrm{M}+\mathrm{H}]^{+}$, calcd. for $\mathrm{C}_{24} \mathrm{H}_{32} \mathrm{~N}_{1} \mathrm{O}_{3} 382.2377$.
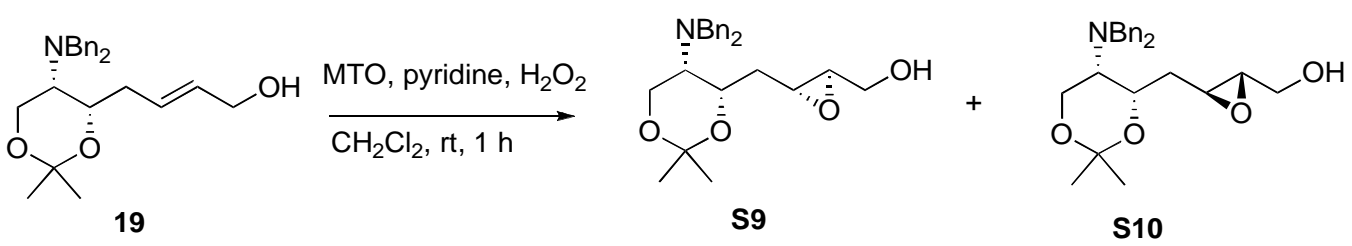

(3-((4S,5S)-5-(dibenzylamino)-2,2-dimethyl-1,3-dioxan-4-yl)methyl)oxiran-2yl)methanol (S9 + S10). To a solution of alkene $19(100 \mathrm{mg}, 262 \mu \mathrm{mol})$ in dichloromethane $(0.4 \mathrm{~mL})$ at $0{ }^{\circ} \mathrm{C}$ was added pyridine $(2.5 \mu \mathrm{L}, 31 \mu \mathrm{mol})$, methyltrioxorhenium $(3.3 \mathrm{mg}, 12 \mu \mathrm{mol})$ and hydrogen peroxide $(40 \mu \mathrm{L}$ of $30 \%$ solution, $393 \mu \mathrm{mol})$. Solution was warmed to room temperature and stirred for 1 hour, then quenched with water $(3 \mathrm{~mL})$. The mixture was extracted with ethyl ether $(4 \times 3 \mathrm{~mL})$ combined extracts washed with brine $(5 \mathrm{~mL})$, dried over $\mathrm{Na}_{2} \mathrm{SO}_{4}$ and concentrated under reduced pressure. Flash chromatography (silica saturated with triethylamine, 1:3 EtOAc:hexane) provided recovered starting material 19 (24.2 mg, 24\%) and an inseparable mixture of S9 and $\mathbf{S 1 0}$ (10.9 $\mathrm{mg}, 14 \%$ adjusted for recovered starting material, dr 1:1 of $\mathbf{S 9 / S 1 0}$ by NMR) as a viscous oil.
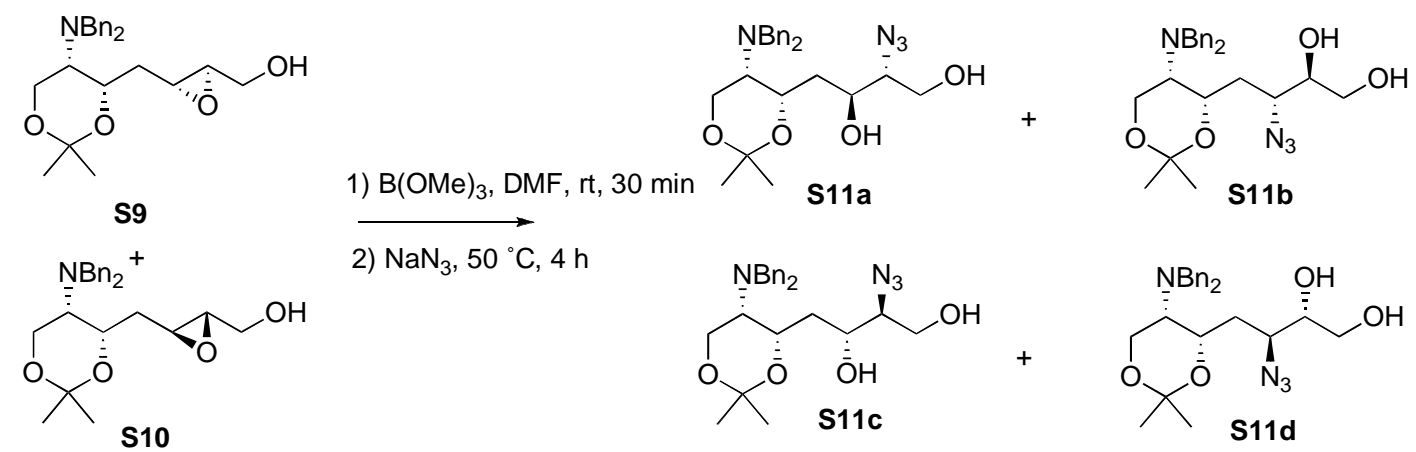

\section{2-azido-4-((4S,5S)-5-(dibenzylamino)-2,2-dimethyl-1,3-dioxan-4-yl)butane-1,3-diol} (S11a-d). Under an atmosphere of nitrogen, $(\mathrm{MeO})_{3} \mathrm{~B}(11.3 \mu \mathrm{L}, 10.4 \mathrm{mg}, 99.6 \mu \mathrm{mol})$ was added to a solution of $\mathbf{S 9}$ and $\mathbf{S 1 0}(18 \mathrm{mg}, 45 \mu \mathrm{mol})$ in anhydrous DMF $(250 \mu \mathrm{L})$. The solution was stirred for $30 \mathrm{~min}$ at room temperature then $\mathrm{NaN}_{3}(6.47 \mathrm{mg}, 99.6 \mu \mathrm{mol})$ was added and the reaction was heated to $50{ }^{\circ} \mathrm{C}$ and stirred for 4 hours. The reaction was cooled to room temperature and quenched by addition of a saturated solution of $\mathrm{NaHCO}_{3}$ 
$(3.0 \mathrm{~mL})$ and the solution stirred a further 30 minutes. The mixture was extracted with ethyl ether $(4 \times 3 \mathrm{~mL})$ and combined extracts washed with brine $(5 \mathrm{~mL})$, dried over $\mathrm{Na}_{2} \mathrm{SO}_{4}$ and concentrated under reduced pressure. Flash chromatography (silica, $40 \%$ ethyl acetate in hexane) provided S11a, S11b, S11c and S11d (12.4 mg, 62\%) as an inseparable mixture.

S11a-d

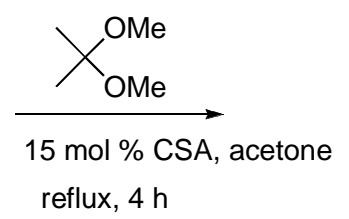

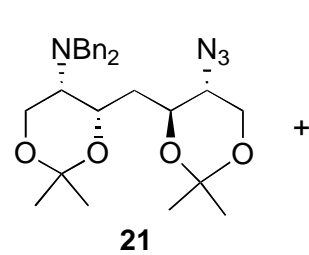

21

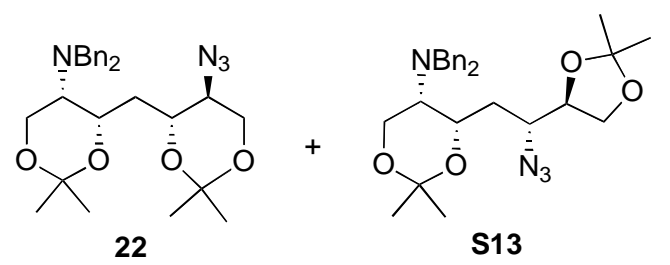

Synthesis of azides 21 and 22. A sealed vial containing a mixture of diols S11a-d (12.0 $\mathrm{mg}, 27 \mu \mathrm{mol})$ and CSA $(0.7 \mathrm{mg}, 2.7 \mu \mathrm{mol})$ in 1:1 2,2-dimethoxypropane:acetone (600 $\mu \mathrm{L})$ was heated at $50{ }^{\circ} \mathrm{C}$ with stirring for 4 hours. The stirred mixture was cooled to room temperature and quenched with saturated aqueous $\mathrm{NaHCO}_{3}(3 \mathrm{~mL})$. The mixture was extracted with ethyl ether $(4 \times 3 \mathrm{~mL})$ and combined extracts washed with brine $(3 \mathrm{~mL})$, dried over $\mathrm{Na}_{2} \mathrm{SO}_{4}$ and concentrated under reduced pressure. Flash chromatography ( silica, 10\% ethyl acetate in hexane) followed by HPLC purification (silica $10 \times 250 \mathrm{~mm}$ column, 1:19 EtOAc:hexane, $3.5 \mathrm{~mL} / \mathrm{min}$ ) provided pure samples of 21, S12, 22 and S13 (10.9 mg, 10:1:40:8 ratio respectively, 84\%). Compound 21, S12, and S13 were viscous oils while compound 22 was a crystalline solid.

(4S,5S)-4-(((4S,5R)-5-azido-2,2-dimethyl-1,3-dioxan-4-yl)methyl)- $N, N$-dibenzyl-2,2dimethyl-1,3-dioxan-5-amine (21). IR (neat) v 2993, 2921, 2853, 2802, 2097, 1494, $1451,1375,1265,1197,1163,1120,1069,1001,967,882,814,746 \mathrm{~cm}^{-1} ;[\alpha]_{\mathrm{D}}{ }^{25}-21.2^{\circ}$ $\left(\mathrm{CHCl}_{3}, c\right.$ 0.94); ${ }^{1} \mathrm{H} \mathrm{NMR}\left(500 \mathrm{MHz}, \mathrm{CDCl}_{3}\right) \delta 7.39(\mathrm{~d}, J=7.5 \mathrm{~Hz}, 4 \mathrm{H}), 7.30(\mathrm{t}, J=7.5$ $\mathrm{Hz}, 4 \mathrm{H}), 7.21(\mathrm{t}, J=7.5 \mathrm{~Hz}, 2 \mathrm{H}), 4.35(\mathrm{~d}, J=13.0 \mathrm{~Hz}, 1 \mathrm{H}), 4.32(\mathrm{bs}, 2 \mathrm{H}), 4.22$ (dt, $J=$ 12.0, $2.0 \mathrm{~Hz}, 1 \mathrm{H}), 3.97(\mathrm{dd}, J=12.0,5.5 \mathrm{~Hz}, 1 \mathrm{H}), 3.92(\mathrm{dd}, J=13.0,3.5 \mathrm{~Hz}, 1 \mathrm{H}), 3.86$ $(\mathrm{td}, J=11.0,2.0 \mathrm{~Hz}, 1 \mathrm{H}), 3.65(\mathrm{dd}, J=11.5,10.0 \mathrm{~Hz}, 1 \mathrm{H}), 3.54(\mathrm{~d}, J=14.0 \mathrm{~Hz}, 2 \mathrm{H})$, $3.30(\mathrm{dt}, J=9.5,5.5 \mathrm{~Hz}, 1 \mathrm{H}), 2.66(\mathrm{ddd}, J=13.5,11.0,2.0 \mathrm{~Hz}, 1 \mathrm{H}), 2.29(\mathrm{t}, J=3.5 \mathrm{~Hz}$, $1 \mathrm{H}), 1.39$ (s, 3H), $1.38(\mathrm{bs}, 6 \mathrm{H}), 1.33(\mathrm{~s}, 3 \mathrm{H}) ;{ }^{13} \mathrm{C} \mathrm{NMR}\left(100 \mathrm{MHz}, \mathrm{CDCl}_{3}\right) \delta 140.6(\mathrm{C})$, $128.8(\mathrm{CH}), 128.5(\mathrm{CH}), 127.0(\mathrm{CH}), 99.1(\mathrm{C}), 98.8(\mathrm{C}), 68.3(\mathrm{CH}), 67.1(\mathrm{CH}), 62.9$ $\left(\mathrm{CH}_{2}\right), 59.9(\mathrm{CH}), 58.6\left(\mathrm{CH}_{2}\right), 56.2\left(\mathrm{CH}_{2}\right), 52.1(\mathrm{CH}), 35.4\left(\mathrm{CH}_{2}\right), 29.7\left(\mathrm{CH}_{3}\right), 28.7$ $\left(\mathrm{CH}_{3}\right), 19.5\left(\mathrm{CH}_{3}\right), 19.0\left(\mathrm{CH}_{3}\right)$; HRFABMS m/z $481.2816[\mathrm{M}+\mathrm{H}]^{+}$, calcd. for $\mathrm{C}_{27} \mathrm{H}_{37} \mathrm{~N}_{4} \mathrm{O}_{4}$ 481.2809.

(4S,5S)-4-(((4R,5S)-5-azido-2,2-dimethyl-1,3-dioxan-4-yl)methyl)- $N, N$-dibenzyl-2,2dimethyl-1,3-dioxan-5-amine (22). IR (neat) v 2989, 2921, 2853, 2106, 1494, 1451, 
$1375,1265,1205,1205,1061,950,746,695 \mathrm{~cm}^{-1} ; \mathrm{mp} 138^{\circ} \mathrm{C} ;[\alpha]_{\mathrm{D}}{ }^{25}+38.0^{\circ}\left(\mathrm{CHCl}_{3}, c\right.$ 2.47); ${ }^{1} \mathrm{H}$ NMR (400 MHz, $\left.\mathrm{CDCl}_{3}\right) \delta 7.45(\mathrm{~d}, J=7.2 \mathrm{~Hz}, 4 \mathrm{H}), 7.32(\mathrm{t}, J=7.2 \mathrm{~Hz}, 4 \mathrm{H})$, $7.23(\mathrm{t}, J=7.2 \mathrm{~Hz}, 2 \mathrm{H}), 4.47(\mathrm{~d}, J=13.2 \mathrm{~Hz}, 1 \mathrm{H}), 4.40-4.26(\mathrm{~m}, 3 \mathrm{H}), 3.98(\mathrm{dd}, J=12.8$, $3.6 \mathrm{~Hz}, 1 \mathrm{H}), 3.91(\mathrm{dd}, J=11.2,4.4 \mathrm{~Hz}, 1 \mathrm{H}), 3.60-3.46(\mathrm{~m}, 3 \mathrm{H}), 3.37(\mathrm{~m}, 2 \mathrm{H}), 2.43$ (ddd, $J=13.2,8.4,1.6 \mathrm{~Hz}, 1 \mathrm{H}), 2.27(\mathrm{t}, J=2.8 \mathrm{~Hz}, 1 \mathrm{H}), 1.80(\mathrm{~m}, 1 \mathrm{H}), 1.48(\mathrm{~s}, 3 \mathrm{H}), 1.40(\mathrm{~s}$, $3 \mathrm{H}), 1.26(\mathrm{~s}, 3 \mathrm{H}), 0.86(\mathrm{~s}, 3 \mathrm{H}) ;{ }^{13} \mathrm{C} \mathrm{NMR}\left(100 \mathrm{MHz}, \mathrm{CDCl}_{3}\right) \delta 140.4(\mathrm{C}), 129.3(\mathrm{CH})$, 128.4 (CH), 127.1 (CH), $98.8(\mathrm{C}), 98.7(\mathrm{C}), 68.8(\mathrm{CH}), 68.1(\mathrm{CH}), 62.8\left(\mathrm{CH}_{2}\right), 59.4$ $(\mathrm{CH}), 58.2\left(\mathrm{CH}_{2}\right), 56.2\left(\mathrm{CH}_{2}\right), 50.4(\mathrm{CH}), 34.5\left(\mathrm{CH}_{2}\right), 29.7\left(\mathrm{CH}_{3}\right), 28.9\left(\mathrm{CH}_{3}\right), 19.0$ $\left(\mathrm{CH}_{3}\right), 18.9\left(\mathrm{CH}_{3}\right)$; HRFABMS $m / z 481.2806[\mathrm{M}+\mathrm{H}]^{+}$, calcd. for $\mathrm{C}_{27} \mathrm{H}_{37} \mathrm{~N}_{4} \mathrm{O}_{4} 481.2809$.

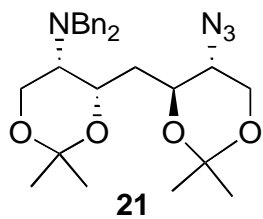

1) $\mathrm{H}_{2}, \mathrm{Pd} / \mathrm{C}, 5: 1 \mathrm{EtOH} / \mathrm{Hexne}$, rt $17 \mathrm{~h}$

2) dry, redisolve $\mathrm{MeOH}, \mathrm{TMSCl}, 1 \mathrm{~h}$

3) dry, redissolve $\mathrm{H}_{2} \mathrm{O}, \mathrm{H}_{2}, \mathrm{Pd} / \mathrm{C}$, rt $18 \mathrm{~h}$

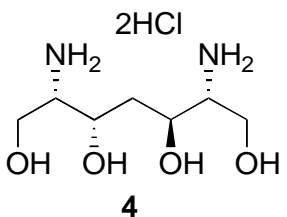

(2R,3S,5S,6S)-2,6-diaminoheptane-1,3,5,7-tetraol (4). A mixture of Pd/C (1.5 mg, 1.3 $\mu \mathrm{mol}, 10 \mathrm{~mol} \% \mathrm{Pd})$ and azide $21(1.8 \mathrm{mg}, 3.7 \mu \mathrm{mol})$ in $5: 1$ ethanol:hexane $(0.5 \mathrm{~mL})$ was placed under $\mathrm{H}_{2}(1 \mathrm{~atm})$ and stirred at room temperature. After 17 hours the mixture was filtered through a $0.45 \mu \mathrm{m}$ syringe filter and concentrated under reduced pressure. The residue was redisolved in dry methanol and $\mathrm{TMSCl}(10.0 \mu \mathrm{L}, 8.5 \mathrm{mg}, 80 \mu \mathrm{mol}) \mathrm{was}$ added and the mixture stirred for 1 hour. The mixture concentrated under reduced pressure. The crude material was resuspended in water $(0.5 \mathrm{~mL})$ and $\mathrm{Pd} / \mathrm{C}(1.5 \mathrm{mg}, 1.3$ $\mu \mathrm{mol}, 10 \mathrm{~mol} \% \mathrm{Pd})$ added. The mixture was placed under $\mathrm{H}_{2}(1 \mathrm{~atm})$ and stirred at room temperature for 18 hours. Filtration through a $0.45 \mu \mathrm{m}$ syringe filter and concentration under reduced pressure provided the hydrochloride salt of $\mathbf{4}(0.8 \mathrm{mg}, 81 \%)$ as a white solid: ${ }^{1} \mathrm{H}$ NMR $\left(400 \mathrm{MHz}, \mathrm{D}_{2} \mathrm{O}\right.$, ref $\left.\mathrm{CH}_{3} \mathrm{CN}\right) \delta 4.05(\mathrm{~m}, 2 \mathrm{H}), 3.85(\mathrm{dd}, J=12.4,4.0 \mathrm{~Hz}$, 2H), $3.73(\mathrm{dd}, J=12.4,7.2 \mathrm{~Hz}, 2 \mathrm{H}), 3.35(\mathrm{~m}, 2 \mathrm{H}), 1.93(\mathrm{dt}, J=14.8,4.0 \mathrm{~Hz}, 1 \mathrm{H}), 1.79$ $(\mathrm{dt}, J=14.8,8.0 \mathrm{~Hz}, 1 \mathrm{H}) ;{ }^{13} \mathrm{C} \mathrm{NMR}\left(100 \mathrm{MHz}, \mathrm{D}_{2} \mathrm{O}, \operatorname{ref} \mathrm{CH}_{3} \mathrm{CN}\right) \delta 65.7(\mathrm{CH}), 65.1$ $(\mathrm{CH}), 59.3\left(\mathrm{CH}_{2}\right), 58.1(\mathrm{CH}), 58.1\left(\mathrm{CH}_{2}\right), 57.4(\mathrm{CH}), 36.5\left(\mathrm{CH}_{2}\right)$; HRESIMS $\mathrm{m} / z$ 195.1339 $[\mathrm{M}+\mathrm{H}]^{+}$, calcd. for $\mathrm{C}_{7} \mathrm{H}_{19} \mathrm{~N}_{2} \mathrm{O}_{4}$ 195.1339.
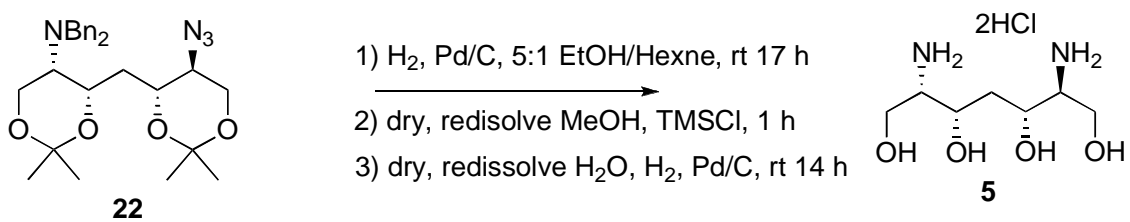

(2R,3R,5S,6S)-2,6-diaminoheptane-1,3,5,7-tetraol (5). A mixture of Pd/C (6.8 mg, 6.4 $\mu \mathrm{mol}, 10 \mathrm{~mol} \% \mathrm{Pd})$ and azide $22(14 \mathrm{mg}, 31.8 \mu \mathrm{mol})$ in methanol $(0.75 \mathrm{~mL})$ was placed under $\mathrm{H}_{2}(1 \mathrm{~atm})$ and stirred at room temperature. After 16 hours TMSCl $(10.0 \mu \mathrm{L}, 8.5$ $\mathrm{mg}, 80 \mu \mathrm{mol}$ ) was added and the mixture stirred a further 1 hour. The mixture was filtered through a $0.45 \mu \mathrm{m}$ syringe filter and concentrated under reduced pressure. The crude material was resuspended in water $(0.5 \mathrm{~mL})$ and $\mathrm{Pd} / \mathrm{C}(6.8 \mathrm{mg}, 6.4 \mu \mathrm{mol}, 10 \mathrm{~mol} \%$ $\mathrm{Pd})$ added. The mixture was placed under $\mathrm{H}_{2}(1 \mathrm{~atm})$ and stirred at room temperature for 14 hours. Filtration through a $0.45 \mu \mathrm{m}$ syringe filter and concentration under reduced 
pressure provided the hydrochloride salt of $\mathbf{5}(8.4 \mathrm{mg}, 99 \%)$ as a white solid: ${ }^{1} \mathrm{H}$ NMR $\left(400 \mathrm{MHz}, \mathrm{D}_{2} \mathrm{O}\right.$, ref ACN) $\delta 4.19(\mathrm{p}, J=4.0 \mathrm{~Hz}, 1 \mathrm{H}), 3.99(\mathrm{~m}, 1 \mathrm{H}), 3.91$ (dd, $J=12.4$, $4.4 \mathrm{~Hz}, 1 \mathrm{H}), 3.85(\mathrm{dd}, J=12.4,4.0 \mathrm{~Hz}, 1 \mathrm{H}), 3.80-3.70(\mathrm{~m}, 2 \mathrm{H}), 3.43$ (apparent p, $J=4.0$ $\mathrm{Hz}, 1 \mathrm{H}), 3.33(\mathrm{~m}, 1 \mathrm{H}), 1.90(\mathrm{dt}, J=14.8,4.4 \mathrm{~Hz}, 1 \mathrm{H}), 1.80(\mathrm{dt}, J=14.8,8.4 \mathrm{~Hz}, 1 \mathrm{H}) ;{ }^{13} \mathrm{C}$ NMR (100 MHz, D $2 \mathrm{O}$, ref ACN) $\delta 67.2(\mathrm{CH}), 66.6(\mathrm{CH}), 59.5\left(\mathrm{CH}_{2}\right), 58.0\left(\mathrm{CH}_{2}\right), 57.5$ $(\mathrm{CH}), 56.6(\mathrm{CH}), 36.2\left(\mathrm{CH}_{2}\right)$; HRESIMS $m / z, 195.1337[\mathrm{M}+\mathrm{H}]^{+}$, calcd. for $\mathrm{C}_{7} \mathrm{H}_{19} \mathrm{~N}_{2} \mathrm{O}_{4}$ 195.1339.

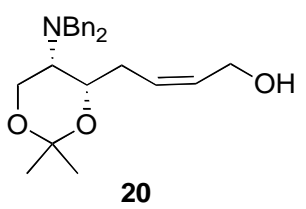

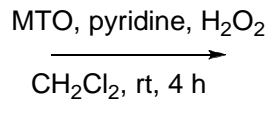

20
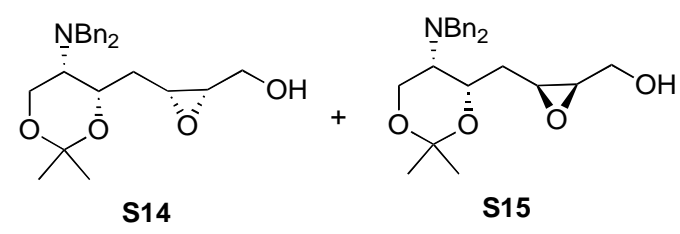

Synthesis of epoxides S14 and S15. To a solution of alkene $20(250 \mathrm{mg}, 655 \mu \mathrm{mol})$ in dichloromethane $(1 \mathrm{~mL})$ at $0{ }^{\circ} \mathrm{C}$ was added pyridine $(10 \mu \mathrm{L}, 124 \mu \mathrm{mol})$, methyltrioxorhenium $(8.2 \mathrm{mg}, 33 \mu \mathrm{mol})$ and hydrogen peroxide $(100 \mu \mathrm{L}$ of $30 \%$ solution, $983 \mu \mathrm{mol})$. Solution was warmed to room temperature and stirred for 4 hours, then quenched by addition of a saturated solution of $\mathrm{NaHCO}_{3}(5 \mathrm{~mL})$. The mixture was extracted with ethyl ether $(4 \times 3 \mathrm{~mL})$ combined extracts washed with brine $(5 \mathrm{~mL})$, dried over $\mathrm{Na}_{2} \mathrm{SO}_{4}$ and concentrated under reduced pressure. Flash chromatography (silica saturated with triethylamine, step gradient of 15, 20, 25, and 30\% ethyl acetate in hexane) provided recovered starting material $20(54.3 \mathrm{mg}, 22 \%)$ and S14 and S15 (30.9 $\mathrm{mg}$ and $17.2 \mathrm{mg}$ respectively, $24 \%$ adjusted for recovered starting material) as a viscous oils.

((2S,3R)-3-(((4S,5S)-5-(dibenzylamino)-2,2-dimethyl-1,3-dioxan-4-yl)methyl)oxiran2-yl)methanol (S14): IR (neat) v 3431, 3026, 2985, 2935, 2869, 2795, 2358, 2333, 1491, 1458, 1384, 1260, 1194, 1070, 947, 740, $699 \mathrm{~cm}^{-1} ;[\alpha]_{\mathrm{D}}{ }^{23}+32.0^{\circ}\left(\mathrm{CHCl}_{3}, c 5.18\right) ;{ }^{1} \mathrm{H}$ NMR $\left(400 \mathrm{MHz}, \mathrm{CDCl}_{3}\right) \delta 7.36(\mathrm{~d}, J=7.2 \mathrm{~Hz}, 4 \mathrm{H}), 7.31(\mathrm{t}, J=7.2 \mathrm{~Hz}, 4 \mathrm{H}), 7.23(\mathrm{t}, J=$ $7.2 \mathrm{~Hz}, 2 \mathrm{H}), 4.41(\mathrm{~d}, J=12.8 \mathrm{~Hz}, 1 \mathrm{H}), 4.30(\mathrm{bs}, 2 \mathrm{H}), 4.17(\mathrm{td}, J=6.8,4.0 \mathrm{~Hz}, 1 \mathrm{H}), 3.98$ $(\mathrm{dd}, J=12.8,3.6 \mathrm{~Hz}, 1 \mathrm{H}), 3.78(\mathrm{dd}, J=12.0,4.4 \mathrm{~Hz}, 1 \mathrm{H}), 3.65(\mathrm{dd}, J=12.0,6.8 \mathrm{~Hz}$, $1 \mathrm{H}), 3.54(\mathrm{~d}, J=13.6 \mathrm{~Hz}, 2 \mathrm{H}), 3.06(\mathrm{td}, J=6.8,4.4 \mathrm{~Hz}, 1 \mathrm{H}), 2.86(\mathrm{~m}, 1 \mathrm{H}), 2.40(\mathrm{t}, J=$ $3.2 \mathrm{~Hz}, 1 \mathrm{H}), 2.08(\mathrm{dt}, J=11.2,5.2 \mathrm{~Hz}, 1 \mathrm{H}), 1.99(\mathrm{dt}, J=11.2,7.2 \mathrm{~Hz}, 1 \mathrm{H}), 1.46(\mathrm{~s}, 3 \mathrm{H})$, $1.40(\mathrm{~s}, 3 \mathrm{H}) ;{ }^{13} \mathrm{C}$ NMR $\left(100 \mathrm{MHz}, \mathrm{CDCl}_{3}\right) \delta 140.2(\mathrm{C}), 128.9(\mathrm{CH}), 128.4(\mathrm{CH}), 127.1$ $(\mathrm{CH}), 99.8(\mathrm{C}), 70.9(\mathrm{CH}), 60.9\left(\mathrm{CH}_{2}\right), 58.3\left(\mathrm{CH}_{2}\right), 56.4(\mathrm{CH}), 56.1\left(\mathrm{CH}_{2}\right), 54.3(\mathrm{CH})$, $50.9(\mathrm{CH}), 30.5\left(\mathrm{CH}_{2}\right), 29.6\left(\mathrm{CH}_{3}\right), 18.8\left(\mathrm{CH}_{3}\right)$; HREIMS $\mathrm{m} / z 397.2245[\mathrm{M}]^{+}$, calcd. for $\mathrm{C}_{24} \mathrm{H}_{31} \mathrm{~N}_{1} \mathrm{O}_{4} 397.2248$.

((2R,3S)-3-(((4S,5S)-5-(dibenzylamino)-2,2-dimethyl-1,3-dioxan-4-yl)methyl)oxiran2-yl)methanol (S15): IR (neat) $v \mathrm{~cm}^{-1} ;[\alpha]_{\mathrm{D}}{ }^{25}-1.4^{\circ}\left(\mathrm{CHCl}_{3}, c 6.40\right) ;{ }^{1} \mathrm{H}$ NMR (400 $\left.\mathrm{MHz} \mathrm{CDCl}_{3}\right) \delta 7.36(\mathrm{~d}, J=7.6 \mathrm{~Hz}, 4 \mathrm{H}), 7.31(\mathrm{t}, J=7.6 \mathrm{~Hz}, 4 \mathrm{H}), 7.23(\mathrm{t}, J=7.6 \mathrm{~Hz}$, $2 \mathrm{H}), 4.38(\mathrm{~d}, J=13.2 \mathrm{~Hz}, 1 \mathrm{H}), 4.28-4.18(\mathrm{~m}, 3 \mathrm{H}), 3.98(\mathrm{dd}, J=13.2,3.6 \mathrm{~Hz}, 1 \mathrm{H}), 3.85$ $(\mathrm{dd}, J=12.0,5.2 \mathrm{~Hz}, 1 \mathrm{H}), 3.52(\mathrm{~d}, J=14.0 \mathrm{~Hz}, 2 \mathrm{H}), 3.45(\mathrm{dd}, J=12.4,8.4 \mathrm{~Hz}, 1 \mathrm{H}), 3.17$ $(\mathrm{p}, J=4.2 \mathrm{~Hz}, 1 \mathrm{H}), 3.00(\mathrm{dt}, J=10.0,4.0 \mathrm{~Hz}, 1 \mathrm{H}), 2.35(\mathrm{t}, J=3.4 \mathrm{~Hz}, 1 \mathrm{H}), 2.21(\mathrm{dt}, J=$ $14.8,10.4 \mathrm{~Hz}, 1 \mathrm{H}), 2.04(\mathrm{ddd}, J=14.8,4.0,2.0 \mathrm{~Hz}, 1 \mathrm{H}), 1.49(\mathrm{~s}, 3 \mathrm{H}), 1.41(\mathrm{~s}, 3 \mathrm{H}) ;{ }^{13} \mathrm{C}$ NMR (100 MHz, $\left.\mathrm{CDCl}_{3}\right) \delta 139.9(\mathrm{C}), 128.6(\mathrm{CH}), 128.4(\mathrm{CH}), 127.0(\mathrm{CH}), 99.4(\mathrm{C})$, 
$70.7(\mathrm{CH}), 60.1\left(\mathrm{CH}_{2}\right), 58.3\left(\mathrm{CH}_{2}\right), 56.1\left(\mathrm{CH}_{2}\right), 55.6(\mathrm{CH}), 55.1(\mathrm{CH}), 51.9(\mathrm{CH}), 30.7$ $\left(\mathrm{CH}_{2}\right), 29.4\left(\mathrm{CH}_{3}\right), 19.1\left(\mathrm{CH}_{3}\right)$; HREIMS $m / z 398.2323[\mathrm{M}+\mathrm{H}]^{+}$, calcd. for $\mathrm{C}_{24} \mathrm{H}_{32} \mathrm{~N}_{1} \mathrm{O}_{4}$ 398.2326 .

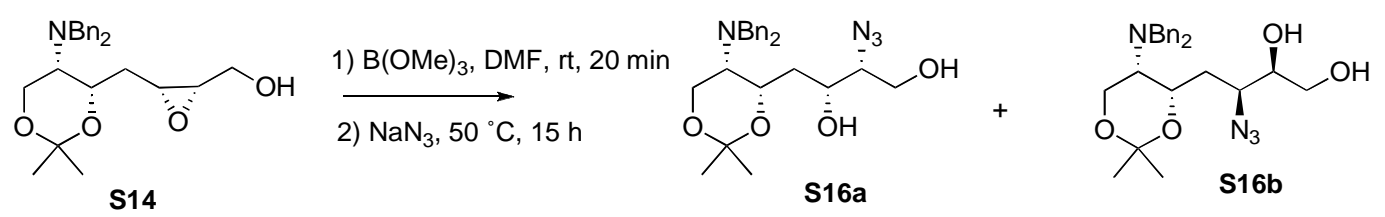

$(2 R, 3 R)$-2-azido-4-((4S,5S)-5-(dibenzylamino)-2,2-dimethyl-1,3-dioxan-4-yl)butane1,3-diol (S16a). Under an atmosphere of nitrogen, $(\mathrm{MeO})_{3} \mathrm{~B}(23.4 \mu \mathrm{L}, 21.4 \mathrm{mg}, 206$ $\mu \mathrm{mol})$ was added to a solution of $\mathbf{S 1 4}(36.9 \mathrm{mg}, 92.8 \mu \mathrm{mol})$ in anhydrous DMF $(600 \mu \mathrm{L})$. The solution was stirred for $20 \mathrm{~min}$ at room temperature then $\mathrm{NaN}_{3}(13.4 \mathrm{mg}, 206 \mu \mathrm{mol})$ was added and the reaction was heated to $50^{\circ} \mathrm{C}$ and stirred for 15 hours. The reaction was cooled to room temperature and quenched by addition of a saturated solution of $\mathrm{NaHCO}_{3}(3.0 \mathrm{~mL})$ and the solution stirred a further 60 minutes. The mixture was extracted with ethyl ether $(4 \times 3 \mathrm{~mL})$ and combined extracts washed with brine $(5 \mathrm{~mL})$, dried over $\mathrm{Na}_{2} \mathrm{SO}_{4}$ and concentrated under reduced pressure. Flash chromatography (silica, 50\% ethyl acetate in hexane) followed by HPLC purification (silica $10 \times 250 \mathrm{~mm}$ column, 8\% isopropanol in hexane, $3.5 \mathrm{~mL} / \mathrm{min}$ ) provided $\mathbf{S 1 6 a}$ and $\mathbf{S 1 6 b}(20.6 \mathrm{mg}$ and $10.8 \mathrm{mg}$ respectively, 77\%) as viscous oils. S16a: IR (neat) $v 3433,2990,2928,2850$, 2104, 1499, 1452, 1383, 1266, 1204, 1150, 1072, $971 \mathrm{~cm}^{-1} ;[\alpha]_{\mathrm{D}}^{23}+4.5^{\circ}\left(\mathrm{CHCl}_{3}, c\right.$ 3.48); ${ }^{1} \mathrm{H}$ NMR $\left(400 \mathrm{MHz}, \mathrm{CDCl}_{3}\right) \delta 7.38(\mathrm{~d}, J=7.6 \mathrm{~Hz}, 4 \mathrm{H}), 7.32(\mathrm{t}, J=7.6 \mathrm{~Hz}, 4 \mathrm{H}), 7.24(\mathrm{t}, J$ $=7.6 \mathrm{~Hz}, 2 \mathrm{H}), 4.38(\mathrm{~d}, J=13.2 \mathrm{~Hz}, 1 \mathrm{H}), 4.29(\mathrm{~m}, 3 \mathrm{H}), 4.00-3.80(\mathrm{~m}, 4 \mathrm{H}), 3.54(\mathrm{~d}, J=$ $13.6 \mathrm{~Hz}, 2 \mathrm{H}), 3.79$ (dt, $J=7.4,4.4 \mathrm{~Hz}, 1 \mathrm{H}), 2.45(\mathrm{dt}, J=14.6,10.0 \mathrm{~Hz}, 1 \mathrm{H}), 3.35(\mathrm{t}, J=$ $3.2 \mathrm{~Hz}, 1 \mathrm{H}), 1.68(\mathrm{dt}, J=14.6,2.4 \mathrm{~Hz}, 1 \mathrm{H}), 1.49(\mathrm{~s}, 3 \mathrm{H}), 1.40(\mathrm{~s}, 3 \mathrm{H}) ;{ }^{13} \mathrm{C}$ NMR $(100$ $\left.\mathrm{MHz}, \mathrm{CDCl}_{3}\right) \delta 140.1(\mathrm{C}), 128.9(\mathrm{CH}), 128.5(\mathrm{CH}), 127.2(\mathrm{CH}), 99.1(\mathrm{C}), 73.4(\mathrm{CH})$, $72.7\left(\mathrm{CH}_{2}\right), 66.8(\mathrm{CH}), 63.2\left(\mathrm{CH}_{2}\right), 58.3\left(\mathrm{CH}_{2}\right), 56.1\left(\mathrm{CH}_{2}\right), 51.5(\mathrm{CH}), 35.4\left(\mathrm{CH}_{2}\right), 29.6$ $\left(\mathrm{CH}_{3}\right), 19.0\left(\mathrm{CH}_{3}\right)$; HRESIMS $m / z 441.2493[\mathrm{M}+\mathrm{H}]^{+}$, calcd. for $\mathrm{C}_{24} \mathrm{H}_{33} \mathrm{~N}_{4} \mathrm{O}_{4} 441.2496$.

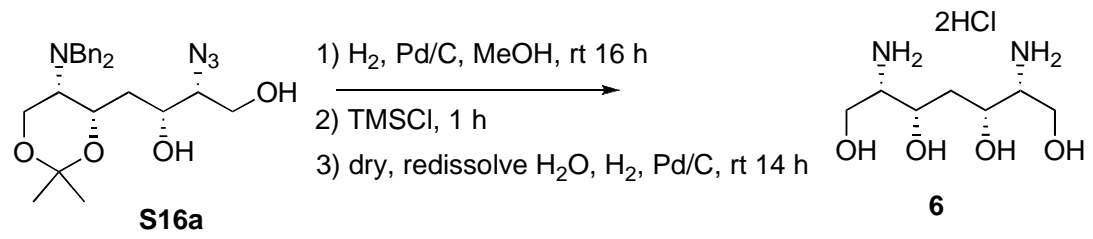

$(2 R, 3 R, 5 S, 6 S)$-2,6-diaminoheptane-1,3,5,7-tetraol (6). A mixture of Pd/C (6.8 mg, 6.4 $\mu \mathrm{mol}, 10 \mathrm{~mol} \% \mathrm{Pd})$ and azide S16a $(14 \mathrm{mg}, 31.8 \mu \mathrm{mol})$ in methanol $(0.75 \mathrm{~mL})$ was placed under $\mathrm{H}_{2}(1 \mathrm{~atm})$ and stirred at room temperature. After 16 hours TMSCl (10.0 $\mu \mathrm{L}, 8.5 \mathrm{mg}, 80 \mu \mathrm{mol}$ ) was added and the mixture stirred a further 1 hour. The mixture was filtered through a $0.45 \mu \mathrm{m}$ syringe filter and concentrated under reduced pressure. The crude material was resuspended in water $(0.5 \mathrm{~mL})$ and $\mathrm{Pd} / \mathrm{C}(6.8 \mathrm{mg}, 6.4 \mu \mathrm{mol}, 10$ $\mathrm{mol} \% \mathrm{Pd})$ added. The mixture was placed under $\mathrm{H}_{2}(1 \mathrm{~atm})$ and stirred at room temperature for 14 hours. Filtration through a $0.45 \mu \mathrm{m}$ syringe filter and concentration under reduced pressure provided the hydrochloride salt of $6(8.4 \mathrm{mg}, 99 \%)$ as a white 
solid: ${ }^{1} \mathrm{H}$ NMR (400 MHz, $\mathrm{D}_{2} \mathrm{O}$, ref internal $\left.\mathrm{CH}_{3} \mathrm{CN}\right) \delta 4.05(\mathrm{~m}, 2 \mathrm{H}), 3.85(\mathrm{dd}, J=12.4$, $4.0 \mathrm{~Hz}, 2 \mathrm{H}), 3.73(\mathrm{dd}, J=12.4,7.2 \mathrm{~Hz}, 2 \mathrm{H}), 3.35(\mathrm{~m}, 2 \mathrm{H}), 1.93(\mathrm{dt}, J=14.8,4.0 \mathrm{~Hz}$, $1 \mathrm{H}), 1.79(\mathrm{dt}, J=14.8,8.0 \mathrm{~Hz}, 1 \mathrm{H}) ;{ }^{13} \mathrm{C} \mathrm{NMR}\left(100 \mathrm{MHz}, \mathrm{D}_{2} \mathrm{O}\right.$, ref internal $\left.\mathrm{CH}_{3} \mathrm{CN}\right) \delta$ $66.3(\mathrm{CH}), 59.5\left(\mathrm{CH}_{2}\right), 57.4(\mathrm{CH}), 36.9\left(\mathrm{CH}_{2}\right)$; HRESIMS $m / z .195 .1337[\mathrm{M}+\mathrm{H}]^{+}$, calcd. for $\mathrm{C}_{7} \mathrm{H}_{19} \mathrm{~N}_{2} \mathrm{O}_{4} 195.1339$.
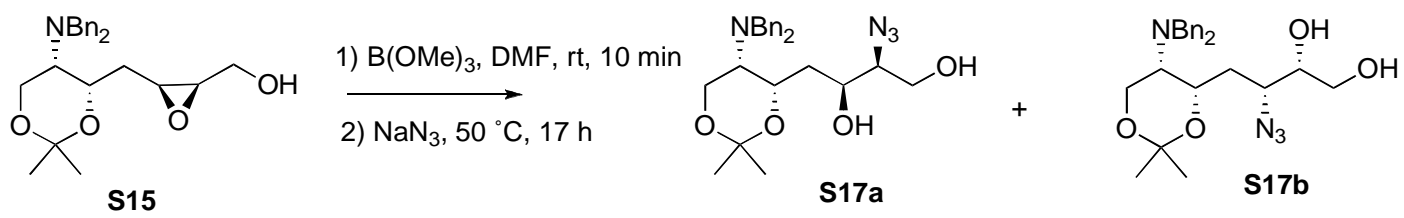

(2S,3S)-2-azido-4-((4S,5S)-5-(dibenzylamino)-2,2-dimethyl-1,3-dioxan-4-yl)butane1,3-diol (S17a). Under an atmosphere of nitrogen, $(\mathrm{MeO})_{3} \mathrm{~B}(9.80 \mu \mathrm{L}, 8.90 \mathrm{mg}, 86 \mu \mathrm{mol})$ was added to a solution of $\mathbf{S 1 5}(17.1 \mathrm{mg}, 43.0 \mu \mathrm{mol})$ in anhydrous DMF $(220 \mu \mathrm{L})$. The solution was stirred for 10 minutes at room temperature then $\mathrm{NaN}_{3}(5.6 \mathrm{mg}, 86 \mu \mathrm{mol})$ was added and the reaction was heated to $50^{\circ} \mathrm{C}$ and stirred for 17 hours. The reaction was cooled to room temperature and quenched by addition of a saturated solution of $\mathrm{NaHCO}_{3}(3.0 \mathrm{~mL})$ and the solution stirred a further 60 minutes. The mixture was extracted with ethyl ether $(4 \times 3 \mathrm{~mL})$ and combined extracts washed with brine $(5 \mathrm{~mL})$, dried over $\mathrm{Na}_{2} \mathrm{SO}_{4}$ and concentrated under reduced pressure. Flash chromatography (silica, $50 \%$ ethyl acetate in hexane) followed by HPLC purification (silica $10 \times 250 \mathrm{~mm}$ column, $8 \%$ isopropanol in hexane, $3.5 \mathrm{~mL} / \mathrm{min})$ provided $\mathbf{S 1 7 a}$ and $\mathbf{S 1 7 b}(3.3 \mathrm{mg}$ and $2.0 \mathrm{mg}$ respectively, 28\%) as viscous oils. S17a: IR (neat) v 3425, 2923, 2851, 2105 , $1493,1452,1198,1093,1069,1027,748,699 \mathrm{~cm}^{-1} ;[\alpha]_{\mathrm{D}}{ }^{22}+27.9^{\circ}\left(\mathrm{CHCl}_{3}, c 1.48\right) ;{ }^{1} \mathrm{H}$ NMR $\left(400 \mathrm{MHz} \mathrm{CDCl}_{3}\right) \delta 7.38(\mathrm{~d}, J=7.2 \mathrm{~Hz}, 4 \mathrm{H}), 7.32(\mathrm{t}, J=7.6 \mathrm{~Hz}, 4 \mathrm{H}), 7.24(\mathrm{t}, J=$ $7.2 \mathrm{~Hz}, 2 \mathrm{H}), 4.38(\mathrm{~d}, J=12.8 \mathrm{~Hz}, 1 \mathrm{H}), 4.29(\mathrm{~m}, 3 \mathrm{H}), 3.97-3.81(\mathrm{~m}, 4 \mathrm{H}), 3.56(\mathrm{~d}, J=14.4$ $\mathrm{Hz}, 2 \mathrm{H}), 3.40(\mathrm{~m}, 1 \mathrm{H}), 2.37(\mathrm{t}, J=2.8 \mathrm{~Hz}, 1 \mathrm{H}), 2.21$ (ddd, $J=14.4,8.8,3.2 \mathrm{~Hz}, 1 \mathrm{H})$, 1.89 (ddd, $J=14.4,9.6,4.8 \mathrm{~Hz}, 1 \mathrm{H}), 1.45$ (s, 3H), 1.38 (s, 3H); ${ }^{13} \mathrm{C}$ NMR $(100 \mathrm{MHz}$, $\left.\mathrm{CDCl}_{3}\right) \delta 139.6(\mathrm{C}), 128.8(\mathrm{CH}), 128.5(\mathrm{CH}), 127.1(\mathrm{CH}), 99.9(\mathrm{C}), 69.7(\mathrm{CH}), 69.6$ $\left(\mathrm{CH}_{2}\right), 67.5(\mathrm{CH}), 64.0\left(\mathrm{CH}_{2}\right), 58.2\left(\mathrm{CH}_{2}\right), 56.2\left(\mathrm{CH}_{2}\right), 51.9(\mathrm{CH}), 37.1\left(\mathrm{CH}_{2}\right), 29.7$ $\left(\mathrm{CH}_{3}\right), 19.1\left(\mathrm{CH}_{3}\right)$; HREIMS $m / z$ 440.2421 [M] $]^{+}$, calcd. for $\mathrm{C}_{24} \mathrm{H}_{32} \mathrm{~N}_{4} \mathrm{O}_{4} 440.2418$.

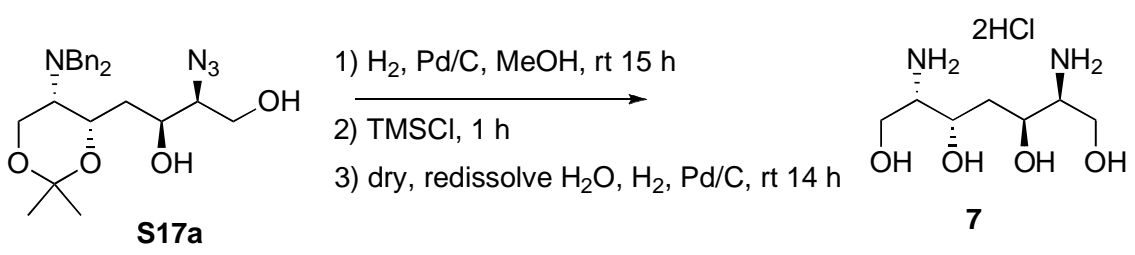

(2S,3S,5S,6S)-2,6-diaminoheptane-1,3,5,7-tetraol (7). A mixture of Pd/C (1.4 mg, 1.4 $\mu \mathrm{mol}, 10 \mathrm{~mol} \% \mathrm{Pd})$ and azide $\mathbf{S 1 7 a}(3.0 \mathrm{mg}, 6.8 \mu \mathrm{mol})$ in methanol $(0.5 \mathrm{~mL})$ was placed under $\mathrm{H}_{2}(1 \mathrm{~atm})$ and stirred at room temperature. After 15 hours TMSCl $(10.0 \mu \mathrm{L}, 8.5$ $\mathrm{mg}, 80 \mu \mathrm{mol})$ was added and the mixture stirred a further 1 hour. The mixture was filtered through a $0.45 \mu \mathrm{m}$ syringe filter and concentrated under reduced pressure. The crude material was resuspended in water $(0.5 \mathrm{~mL})$ and $\mathrm{Pd} / \mathrm{C}(1.4 \mathrm{mg}, 1.4 \mu \mathrm{mol}, 10 \mathrm{~mol} \%$ $\mathrm{Pd})$ added. The mixture was placed under $\mathrm{H}_{2}(1 \mathrm{~atm})$ and stirred at room temperature for 
14 hours. Filtration through a $0.45 \mu \mathrm{m}$ syringe filter and concentration under reduced pressure provided the hydrochloride salt of $\mathbf{7}(1.8 \mathrm{mg}, 99 \%)$ as a white solid: ${ }^{1} \mathrm{H}$ NMR $\left(400 \mathrm{MHz}, \mathrm{D}_{2} \mathrm{O}, \operatorname{ref} \mathrm{CH}_{3} \mathrm{CN}\right) \delta 4.05(\mathrm{~m}, 2 \mathrm{H}), 3.88(\mathrm{dd}, J=12.4,3.6 \mathrm{~Hz}, 2 \mathrm{H}), 3.74(\mathrm{dd}, J$ $=12.4,6.8 \mathrm{~Hz}, 2 \mathrm{H}), 3.30(\mathrm{~m}, 2 \mathrm{H}), 1.75$ (apparent dd, $J=7.6,5.2 \mathrm{~Hz}, 2 \mathrm{H}) ;{ }^{13} \mathrm{C}$ NMR $(100$ $\mathrm{MHz}, \mathrm{D}_{2} \mathrm{O}$, ref $\left.\mathrm{CH}_{3} \mathrm{CN}\right) \delta 64.9(\mathrm{CH}), 59.3\left(\mathrm{CH}_{2}\right), 58.1(\mathrm{CH}), 37.3\left(\mathrm{CH}_{2}\right) ; \operatorname{HRESIMS~} \mathrm{m} / z$ 195.1330 [M+H] $]^{+}$, calcd. for $\mathrm{C}_{7} \mathrm{H}_{19} \mathrm{~N}_{2} \mathrm{O}_{4}$ 195.1339.

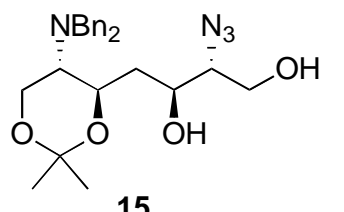

15
3:1 $\mathrm{MeOH}: \mathrm{AcOH}$

$70{ }^{\circ} \mathrm{C}, 15 \mathrm{~h}$

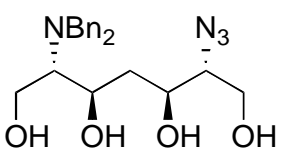

S18

(2R,3S,5R,6S)-2-azido-6-(dibenzylamino)heptane-1,3,5,7-tetraol (S18). Compound 15 $(13.0 \mathrm{mg}, 29.5 \mu \mathrm{mol})$ in methanol:acetic acid 3:1 $(900 \mu \mathrm{L})$ was heated to $70^{\circ} \mathrm{C}$. The mixture was stirred for 23 hours then concentrated under reduced pressure. Flash chromatography (silica, 1:1 ethyl acetate:hexane then 10\% methanol in chloroform) provided S18 (11.2 $\mathrm{mg}, 95 \%$ ) as a viscous oil: IR (neat) $v$ 3371, 3023, 2921, 2794, 2097, $1494,1451,1367,1307,1265,1112,1061,1018.848,746,704 \mathrm{~cm}^{-1} ;[\alpha]_{\mathrm{D}}^{25}+0.6^{\circ}$ $\left(\mathrm{CHCl}_{3}, c\right.$ 2.19); ${ }^{1} \mathrm{H}$ NMR $\left(500 \mathrm{MHz}, \mathrm{CDCl}_{3}\right) \delta 7.34-7.24(\mathrm{~m}, 10 \mathrm{H}), 4.15$ (ddd, $J=10.0$, 8.5, 1.5 Hz, 1H), $4.04(\mathrm{dd}, J=11.0,5.0 \mathrm{~Hz}, 1 \mathrm{H}), 3.97(\mathrm{dd}, J=11.0,6.5 \mathrm{~Hz}, 1 \mathrm{H}), 3.91$ (ddd, $J=10.0,6.0,1.5 \mathrm{~Hz}, 1 \mathrm{H}), 3.86-3.80(\mathrm{~m}, 4 \mathrm{H}), 3.61(\mathrm{~d}, J=13.5 \mathrm{~Hz}, 2 \mathrm{H}), 3.33(\mathrm{q}, J$ $=5.0 \mathrm{~Hz}, 1 \mathrm{H}), 2.66(\mathrm{q}, J=6.0 \mathrm{~Hz}, 1 \mathrm{H}), 2.21(\mathrm{~d}, J=14.5 \mathrm{~Hz}, 1 \mathrm{H}), 1.30(\mathrm{~m}, 1 \mathrm{H}) ;{ }^{13} \mathrm{C}$ NMR (100 MHz, $\left.\mathrm{CDCl}_{3}\right) \delta 139.5(\mathrm{C}), 129.1(\mathrm{CH}), 128.6(\mathrm{CH}), 127.4(\mathrm{CH}), 74.0(\mathrm{CH})$, $73.5(\mathrm{CH}), 66.8,62.8\left(\mathrm{CH}_{2}\right), 62.7(\mathrm{CH}), 59.8\left(\mathrm{CH}_{2}\right), 55.1\left(\mathrm{CH}_{2}\right), 37.8\left(\mathrm{CH}_{2}\right)$; HRFABMS $m / z, 401.2190[\mathrm{M}+\mathrm{H}]^{+}$, calcd. for $\mathrm{C}_{21} \mathrm{H}_{29} \mathrm{~N}_{4} \mathrm{O}_{4} 401.2183$.

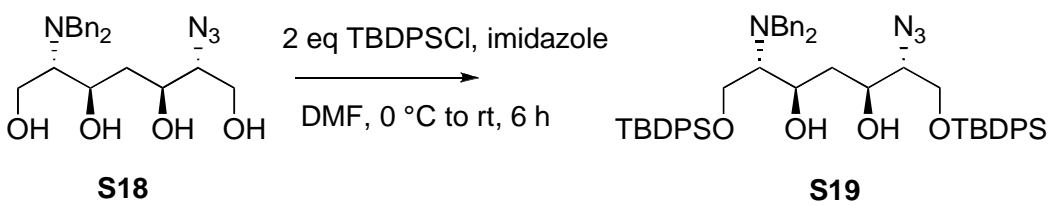

$(6 R, 7 S, 9 R, 10 S)-6-a z i d o-10-($ dibenzylamino)-2,2,14,14-tetramethyl-3,3,13,13tetraphenyl-4,12-dioxa-3,13-disilapentadecane-7,9-diol (S19). Under an atmosphere of nitrogen tert-butyldiphenylchlorosilane $(13.5 \mu \mathrm{L}, 51.9 \mu \mathrm{mol})$ was added to a stirred solution of tetraol S18 $(10.4 \mathrm{mg}, 26.0 \mu \mathrm{mol})$ and imidazole $(4.9 \mathrm{mg}, 68 \mu \mathrm{mol})$ in dimethylformamide $(130 \mu \mathrm{L})$ at room temperature. The mixture was stirred for 2 hours then quenched by addition of water $(5 \mathrm{~mL})$. The mixture was extracted with ethyl ether (4 $\times 3 \mathrm{~mL})$ and combined extracts washed with brine $(5 \mathrm{~mL})$, dried over $\mathrm{Na}_{2} \mathrm{SO}_{4}$ and concentrated under reduced pressure. Flash chromatography (silica, 10\% ethyl acetate in hexane) provided S19 $(17.3 \mathrm{mg}, 76 \%)$ as a viscous oil: IR (neat) $v 3465,3066,3023$, 2930, 2853, 2097, 1468, 1434, 1265, 1112, 814, 746, 704, $610 \mathrm{~cm}^{-1} ;[\alpha]_{\mathrm{D}}^{24}+3.1^{\circ}\left(\mathrm{CHCl}_{3}\right.$, c 5.46); ${ }^{1} \mathrm{H}$ NMR (400 MHz, $\left.\mathrm{CDCl}_{3}\right) \delta$ 7.76-7.66 (m, 8H), 7.52-7.36 (m, 12H), 7.28-7.16 $(\mathrm{m}, 10 \mathrm{H}), 4.16-4.02(\mathrm{~m}, 4 \mathrm{H}), 3.93(\mathrm{dd}, J=10.4,3.6 \mathrm{~Hz}, 1 \mathrm{H}), 3.88(\mathrm{~d}, J=3.6 \mathrm{~Hz}, 1 \mathrm{H})$, $3.82-3.76(\mathrm{~m}, 4 \mathrm{H}), 3.46-3.38(\mathrm{~m}, 3 \mathrm{H}), 2.74(\mathrm{q}, J=5.6 \mathrm{~Hz}, 1 \mathrm{H}), 2.21(\mathrm{~d}, J=14.8 \mathrm{~Hz}, 1 \mathrm{H})$, $1.32-1.20(\mathrm{~m}, 1 \mathrm{H}), 1.09(\mathrm{~s}, 9 \mathrm{H}), 1.06(\mathrm{~s}, 9 \mathrm{H}) ;{ }^{13} \mathrm{C} \mathrm{NMR}\left(100 \mathrm{MHz}, \mathrm{CDCl}_{3}\right) \delta 139.5(\mathrm{C})$, 
$135.8(\mathrm{CH}), 137.7(\mathrm{CH}), 133.3(\mathrm{C}), 133.2(\mathrm{C}), 132.6(\mathrm{C}), 132.5(\mathrm{C}), 130.3(\mathrm{CH}), 130.2$ $(\mathrm{CH}), 130.0(\mathrm{CH}), 128.9(\mathrm{CH}), 128.5(\mathrm{CH}), 128.8(\mathrm{CH}), 127.9(\mathrm{CH}), 127.3(\mathrm{CH}), 74.1$ $(\mathrm{CH}), 72.3(\mathrm{CH}), 68.2(\mathrm{CH}), 64.5\left(\mathrm{CH}_{2}\right), 62.0(\mathrm{CH}), 61.9\left(\mathrm{CH}_{2}\right), 55.4\left(\mathrm{CH}_{2}\right), 37.2\left(\mathrm{CH}_{2}\right)$, $27.0\left(\mathrm{CH}_{3}\right), 26.9\left(\mathrm{CH}_{3}\right), 19.3(\mathrm{C}), 19.2(\mathrm{C})$; HRFABMS $m / 2877.4553[\mathrm{M}+\mathrm{H}]^{+}$, calcd. for $\mathrm{C}_{53} \mathrm{H}_{65} \mathrm{~N}_{4} \mathrm{O}_{4} \mathrm{Si}_{2} 877.4539$.
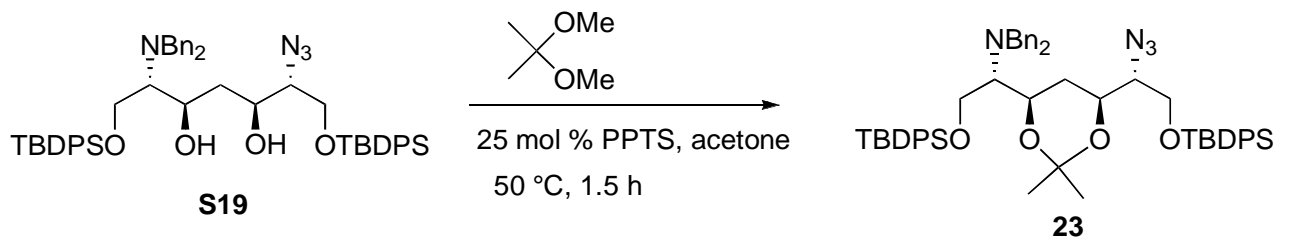

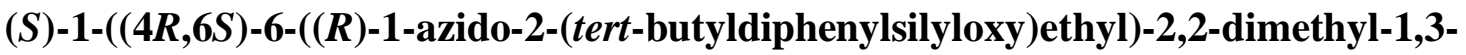
dioxan-4-yl)- $N, N$-dibenzyl-2-(tert-butyldiphenylsilyloxy)ethanamine (23). A sealed vial containing diol $\mathbf{S 1 9}(17.0 \mathrm{mg}, 19.4 \mu \mathrm{mol})$ and PPTS $(2.4 \mathrm{mg}, 9.7 \mu \mathrm{mol})$ in 1:1 2,2dimethoxypropane:acetone $(1 \mathrm{~mL})$ was heated at $50{ }^{\circ} \mathrm{C}$ with stirring for 1.5 hours. The stirred mixture was cooled to room temperature and quenched with saturated aqueous $\mathrm{NaHCO}_{3}(5 \mathrm{~mL})$. The mixture was extracted with ethyl ether $(4 \times 3 \mathrm{~mL})$ and combined extracts washed with brine $(5 \mathrm{~mL})$, dried over $\mathrm{Na}_{2} \mathrm{SO}_{4}$ and concentrated under reduced pressure. Flash chromatography (silica, 1:19 EtOAc:hexane) provided $\mathbf{2 3}(17.2 \mathrm{mg}, 97 \%)$ as a viscous oil: IR (neat) $v \mathrm{~cm}^{-1} ;[\alpha]_{\mathrm{D}}{ }^{25}+6.5^{\circ}\left(\mathrm{CHCl}_{3}, c 6.44\right) ;{ }^{1} \mathrm{H}$ NMR $(400 \mathrm{MHz}$, $\left.\mathrm{CDCl}_{3}\right) \delta 7.70-7.80(\mathrm{~m}, 8 \mathrm{H}), 7.38-7.48(\mathrm{~m}, 12 \mathrm{H}), 7.36-7.20(\mathrm{~m}, 10 \mathrm{H}), 4.22$ (ddd, $J=$ $11.6,7.6,2.4 \mathrm{~Hz}, 1 \mathrm{H}), 4.06-3.92(\mathrm{~m}, 5 \mathrm{H}), 3.81(\mathrm{~d}, J=4.4 \mathrm{~Hz}, 2 \mathrm{H}), 3.71(\mathrm{~d}, J=14.0 \mathrm{~Hz}$, $2 \mathrm{H}), 2.80(\mathrm{dt}, J=7.2,4,4 \mathrm{~Hz}, 1 \mathrm{H}), 2.65(\mathrm{~m}, 1 \mathrm{H}), 2.01(\mathrm{dt}, J=13.2,2.0 \mathrm{~Hz}, 1 \mathrm{H}), 1.32(\mathrm{~s}$, $3 \mathrm{H}), 1.24(\mathrm{~s}, 3 \mathrm{H}), 1.11(\mathrm{~s}, 9 \mathrm{H}), 1.08(\mathrm{~s}, 9 \mathrm{H}) ;{ }^{1} \mathrm{H}$ NMR $\left(400 \mathrm{MHz}, 1: 1 \mathrm{CDCl}_{3}: \mathrm{C}_{6} \mathrm{D}_{6}\right) \delta$ 7.74-7.64 (m, 8H), 7.32-7.24 (m, 12H), 7.22-7.14 (m, 8H), 7.12-7.06 (m, 2H), 4.14 (ddd, $J=10.4,8.0,2.4 \mathrm{~Hz}, 1 \mathrm{H}), 4.01(\mathrm{dd}, J=10.8,2.4 \mathrm{~Hz}, 1 \mathrm{H}), 3.96-3.90(\mathrm{~m}, 3 \mathrm{H}), 3.83$ (ddd, $J=11.6,6.4,2.4 \mathrm{~Hz}, 1 \mathrm{H}), 3.74(\mathrm{~d}, J=4.4 \mathrm{~Hz}, 2 \mathrm{H}), 3.66(\mathrm{~d}, J=13.6 \mathrm{~Hz}, 2 \mathrm{H}), 3.17(\mathrm{dt}, J$ = 9.6, $5.2 \mathrm{~Hz}, 1 \mathrm{H}), 2.68-2.63(\mathrm{~m}, 1 \mathrm{H}), 1.98(\mathrm{~d}, J=9.2 \mathrm{~Hz}, 1 \mathrm{H}), 1.20(\mathrm{~s}, 3 \mathrm{H}), 1.19(\mathrm{~s}, 3 \mathrm{H})$, $1.08(\mathrm{~s}, 9 \mathrm{H}), 1.06(\mathrm{~s}, 9 \mathrm{H}) ;{ }^{13} \mathrm{C} \mathrm{NMR}\left(100 \mathrm{MHz}, \mathrm{CDCl}_{3}\right) \delta 140.4(\mathrm{C}), 135.9(\mathrm{CH}), 135.8$ $(\mathrm{CH}), 135.74(\mathrm{CH}), 135.71(\mathrm{CH}), 133.6(\mathrm{C}), 133.5(\mathrm{C}), 133.2(\mathrm{C}), 133.1(\mathrm{C}), 129.9(\mathrm{CH})$, $129.8(\mathrm{CH}), 129.7(\mathrm{CH}), 128.8(\mathrm{CH}), 128.4(\mathrm{CH}), 127.9(\mathrm{CH}), 127.8(\mathrm{CH}), 127.7(\mathrm{CH})$, 127.0 (CH), 98.8 (C), $68.0(\mathrm{CH}), 67.2(\mathrm{CH}), 67.1(\mathrm{CH}), 63.2\left(\mathrm{CH}_{2}\right), 62.9(\mathrm{CH}), 59.2$ $\left(\mathrm{CH}_{2}\right), 55.9\left(\mathrm{CH}_{2}\right), 32.1\left(\mathrm{CH}_{2}\right), 29.9\left(\mathrm{CH}_{3}\right), 27.1\left(\mathrm{CH}_{3}\right), 26.8\left(\mathrm{CH}_{3}\right), 19.7\left(\mathrm{CH}_{3}\right), 19.3(\mathrm{C})$, $19.2(\mathrm{C})$; HRMS $m / z$ [M+H] $]^{+}$, calcd. for $\mathrm{C}_{53} \mathrm{H}_{65} \mathrm{~N}_{4} \mathrm{O}_{4} \mathrm{Si}_{2}$. 
Table S1. ${ }^{13} \mathrm{C}$ NMR data for 2-7 and Zwittermicin A $\left(\mathbf{1}, 400 \mathrm{MHz}, \mathrm{D}_{2} \mathrm{O}\right)^{\mathrm{a}}$

\begin{tabular}{|c|c|c|c|c|c|c|c|c|c|}
\hline & \multicolumn{9}{|c|}{$\delta_{\mathrm{C}}$} \\
\hline C\# & 2 & 3 & 4 & $4 \mathrm{~b}$ & 5 & $5 b$ & 6 & 7 & $\mathrm{Zw} \mathrm{A} \mathrm{(1)}$ \\
\hline 9 & 58.0 & 58.0 & 59.3 & 58.1 & 59.5 & 58.0 & 59.5 & 59.3 & \\
\hline 10 & 57.3 & 56.6 & 58.1 & 57.4 & 57.5 & 56.6 & 57.4 & 58.1 & 58.3 \\
\hline 11 & 65.9 & 67.4 & 65.1 & 65.7 & 66.6 & 67.2 & 66.3 & 64.9 & 66.0 \\
\hline 12 & 35.8 & 35.3 & 36.5 & 36.5 & 36.2 & 36.2 & 36.9 & 37.3 & 35.4 \\
\hline 13 & 65.9 & 67.4 & 65.7 & 65.1 & 67.2 & 66.6 & 66.3 & 64.9 & 66.1 \\
\hline 14 & 57.3 & 56.6 & 57.4 & 58.1 & 56.6 & 57.5 & 57.4 & 58.1 & 57.4 \\
\hline 15 & 58.0 & 58.0 & 58.1 & 59.3 & 58.0 & 59.5 & 59.5 & 59.3 & 58.1 \\
\hline
\end{tabular}

a. ${ }^{13} \mathrm{C}$ NMR spectra referenced to internal $\mathrm{CH}_{3} \mathrm{CN}(\delta 1.47 \mathrm{ppm})$. For ease of comparison, carbons are numbered with respect to zwittermicin A (1). 


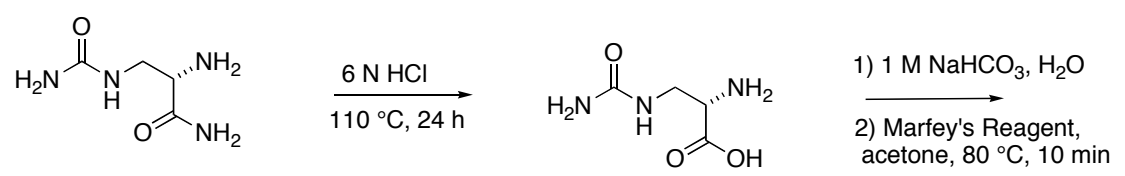

$(-)$-albizziin

3) $1 \mathrm{M} \mathrm{HCl}$

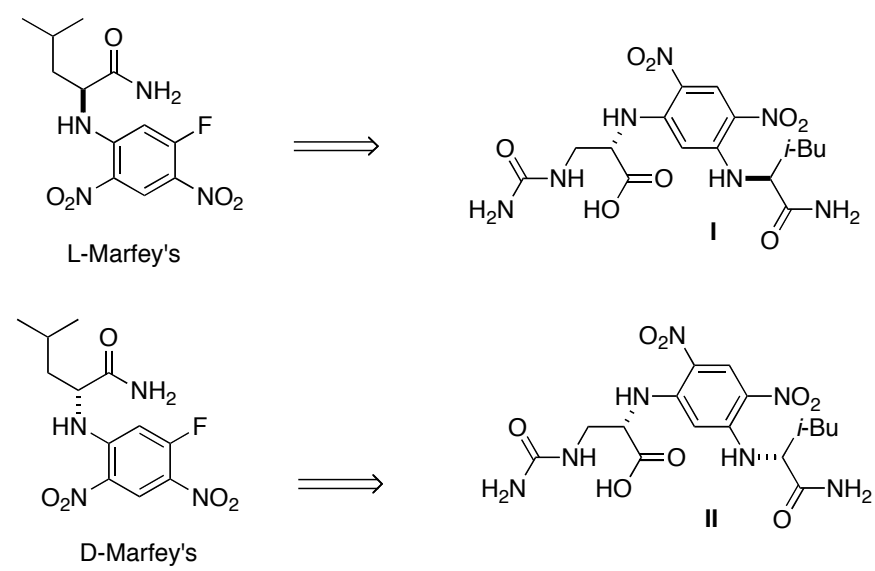

\section{Determination of configuration of $\mathbf{C 4}$ in Zwittermicin (1).}

A solution of $1(148 \mu \mathrm{g})$ in $50 \mu \mathrm{L}$ water and $6 \mathrm{~N} \mathrm{HCl}(1 \mathrm{~mL})$ was heated in a sealed tube at $110{ }^{\circ} \mathrm{C}$ for 24 hours. The solution was concentrated to dryness under a $\mathrm{N}_{2}$ stream to and the hydrolysate redissolved in $1.0 \mathrm{~mL}$ of $\mathrm{H}_{2} \mathrm{O}$.

Marfey's Method. The above hydrolysate solution $(100 \mu \mathrm{L})$ was treated with a solution of 2,4-dinitrophenyl-5-fluoro-L-leucinamide (100 $\mu \mathrm{L}, 1 \% \mathrm{w} / \mathrm{v}$ in acetone), or its enantiomer 2,4-dinitrophenyl-5-fluoro-D-leucinamide, followed by $1.0 \mathrm{M} \mathrm{NaHCO}_{3}(20$ $\mu \mathrm{L}$ ), then heated in a sealed tube at $80^{\circ} \mathrm{C}$ for $10 \mathrm{~min}$. The mixture was cooled and quenched with $1.0 \mathrm{M} \mathrm{HCl}(20 \mu \mathrm{L})$. The preceding paired derivatization procedure was applied to authentic (2S)-(-)-albizziin (Sigma-Aldrich).

$L C$ Analysis. The solutions from Marfey's method were analysed by LC-MS using an Agilent series 1100 HPLC with a Phenomonex Luna C-18 colunm $(100 \mathrm{~mm}$ x $2.00 \mathrm{~mm}$, $3 \mu \mathrm{m})$ connected to a Thermo Finnigan MSQ. LC parameters were as follows; Flow rate $0.40 \mathrm{~mL} / \mathrm{min}$, initial $90 \%$ solvent $\mathrm{A}\left(\mathrm{H}_{2} \mathrm{O}+0.1 \%\right.$ formic acid $) 10 \%$ solvent $\mathrm{B}$ (acetonitrile), @ 15 min 70\% A, @ 20 min 100\% B hold for 5 min, @ 28 min 90\%, A hold for $2 \mathrm{~min}$. Injection volume was $6 \mu \mathrm{L}$. MSQ parameters were as follows; ESI-MS, selected ion monitoring at $\mathrm{m} / z 400[\mathrm{M}+\mathrm{H}]^{+}$, span $2.0 \mathrm{amu}$, dwell $1.00 \mathrm{sec}$, cone $90 \mathrm{~V}$, probe temperature $350{ }^{\circ} \mathrm{C}$. Retention times for the two peaks were $t_{\mathrm{R}}=14.15 \mathrm{~min}$ and $t_{\mathrm{R}}=14.75 \mathrm{~min}$ for the "L-Marfey's-(-)-albizziin" (I) and "D-Marfey's-(-)-albizziin" (II) products, respectively.

The L-Marfey's derivative of the hydrolysate from 1 had a retention time of $t_{\mathrm{R}}=14.13$ min. Coinjection of this sample with $\mathbf{I}$ showed a single peak with retention time of 14.15 min indicating an $S$ configuration for the $N^{3}$-ureido-2,3-diaminopropionic acid residue in 1. 


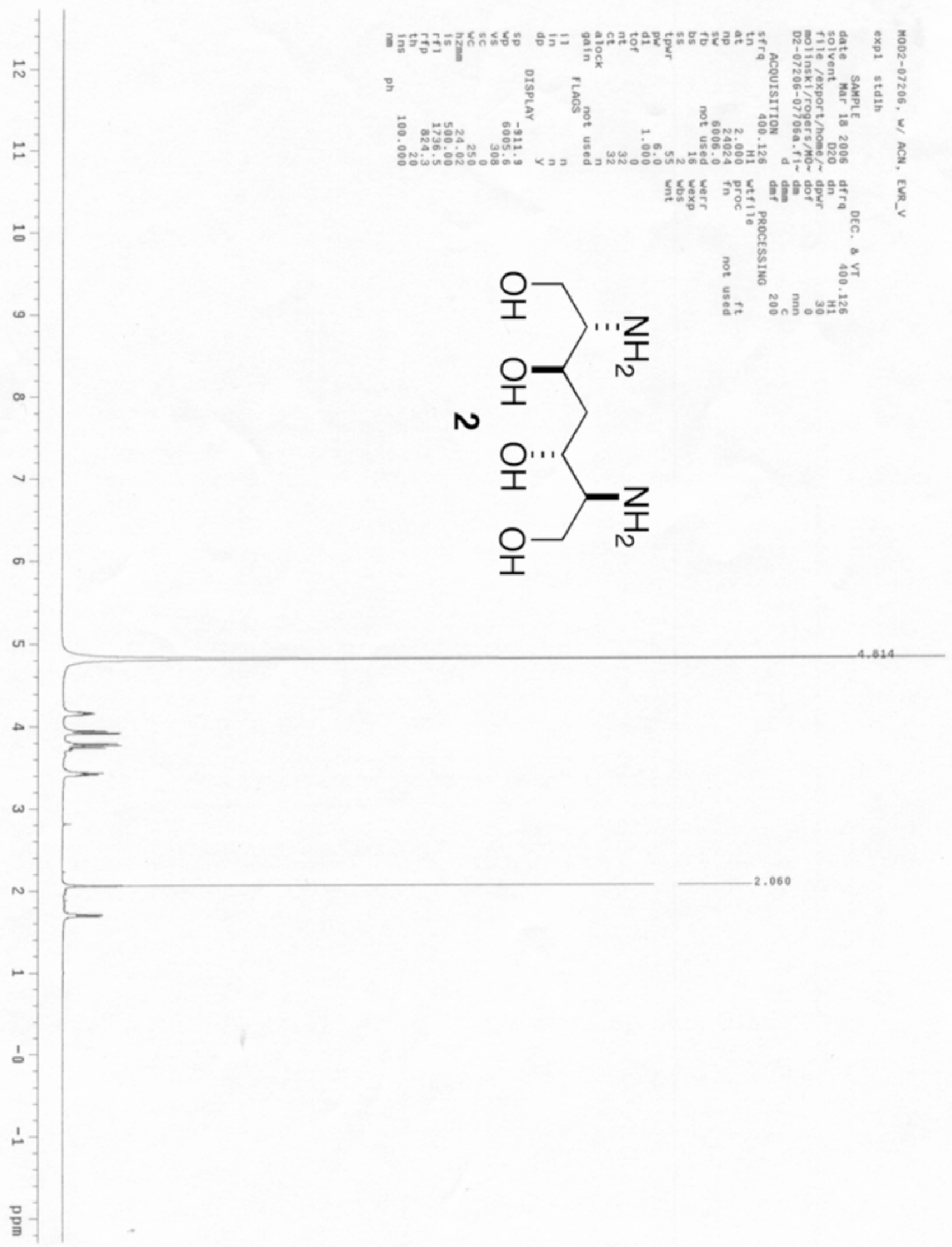




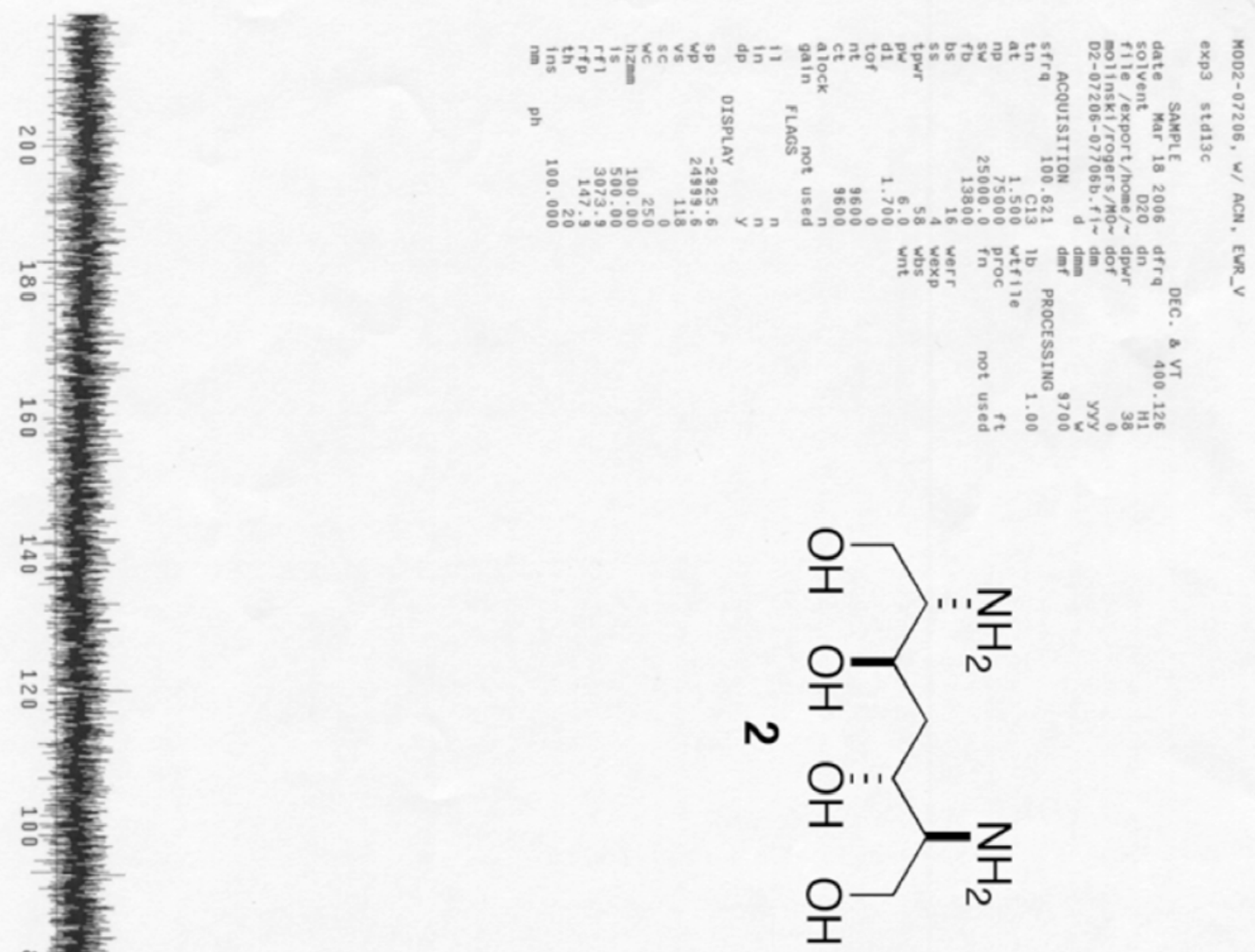



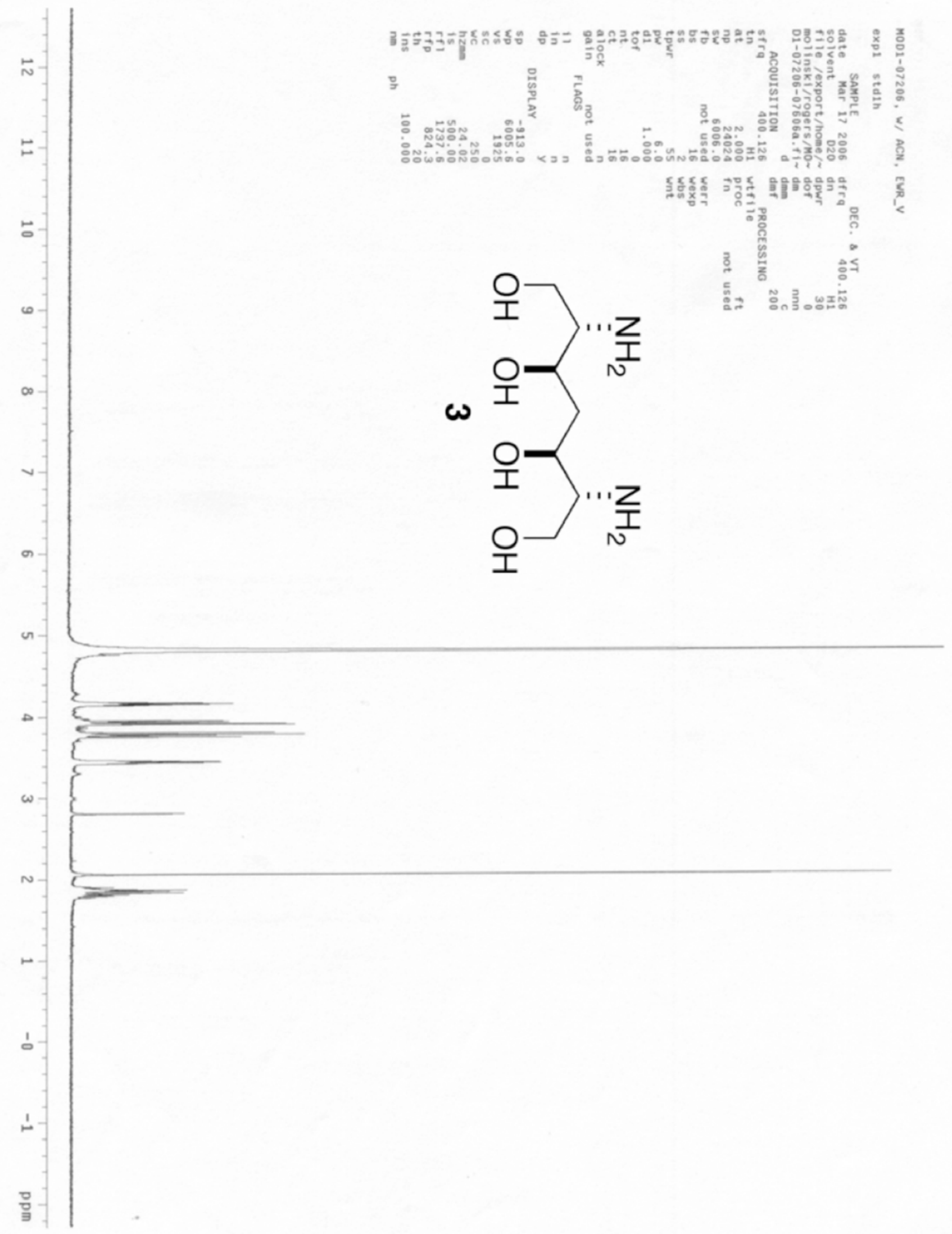


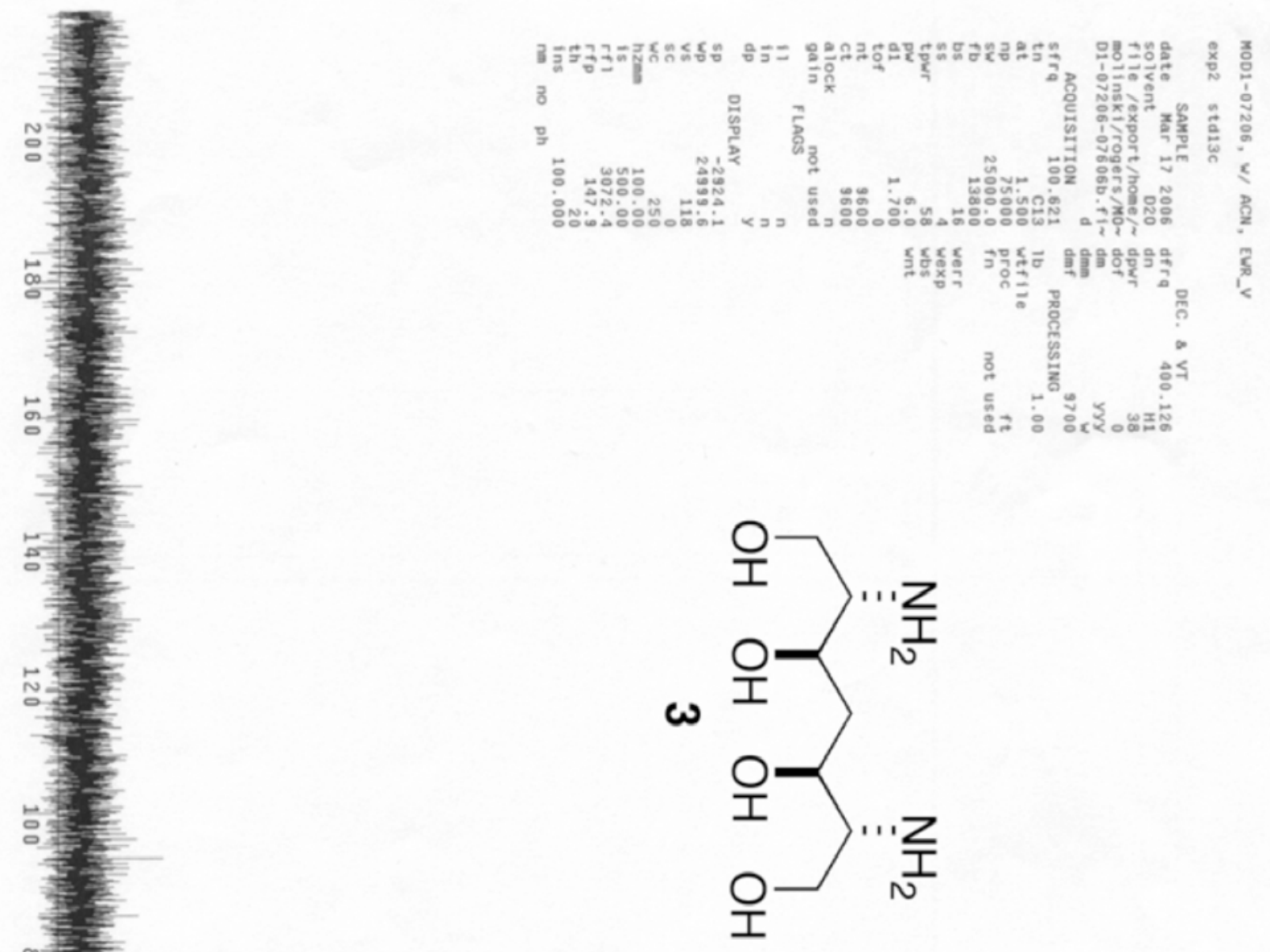



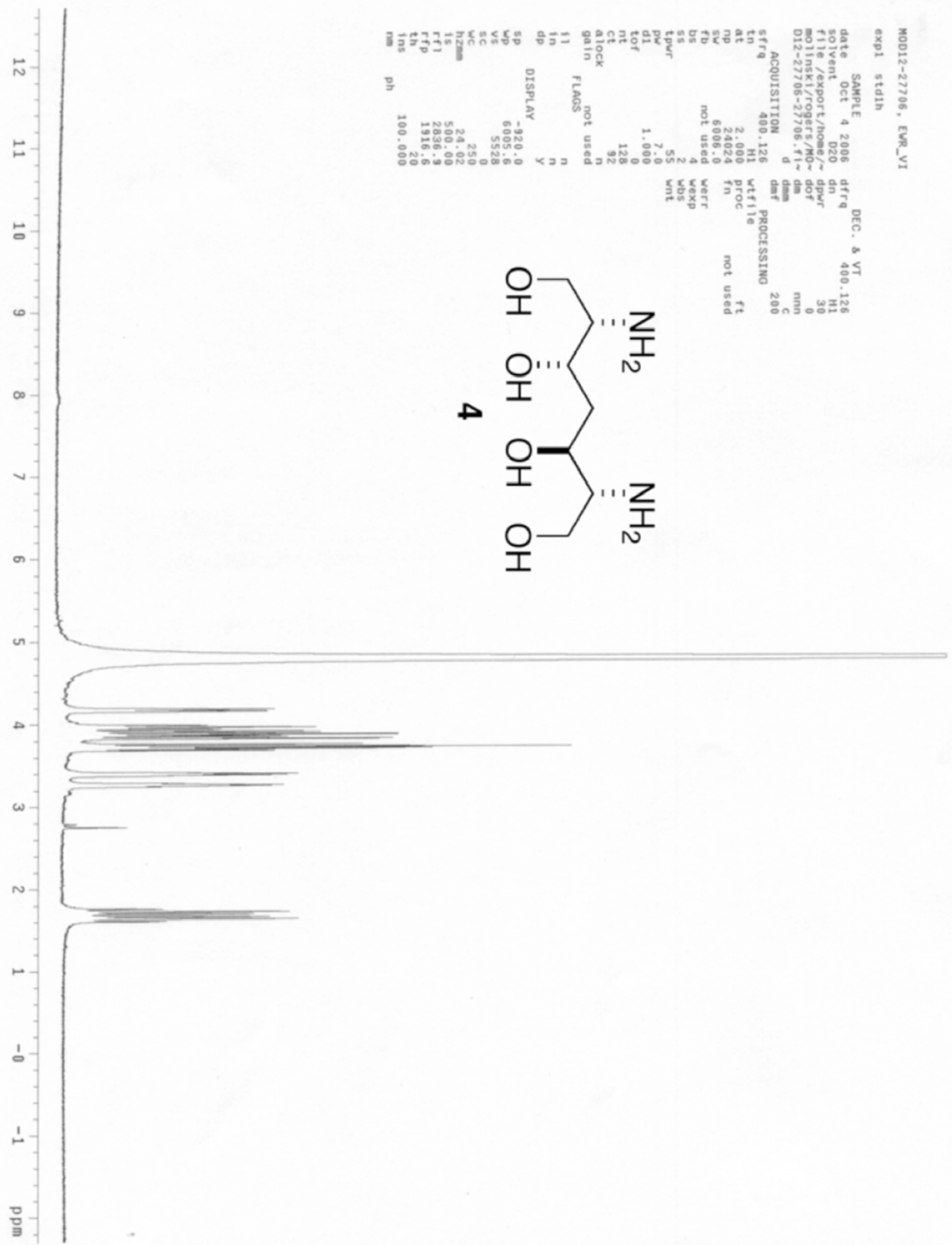


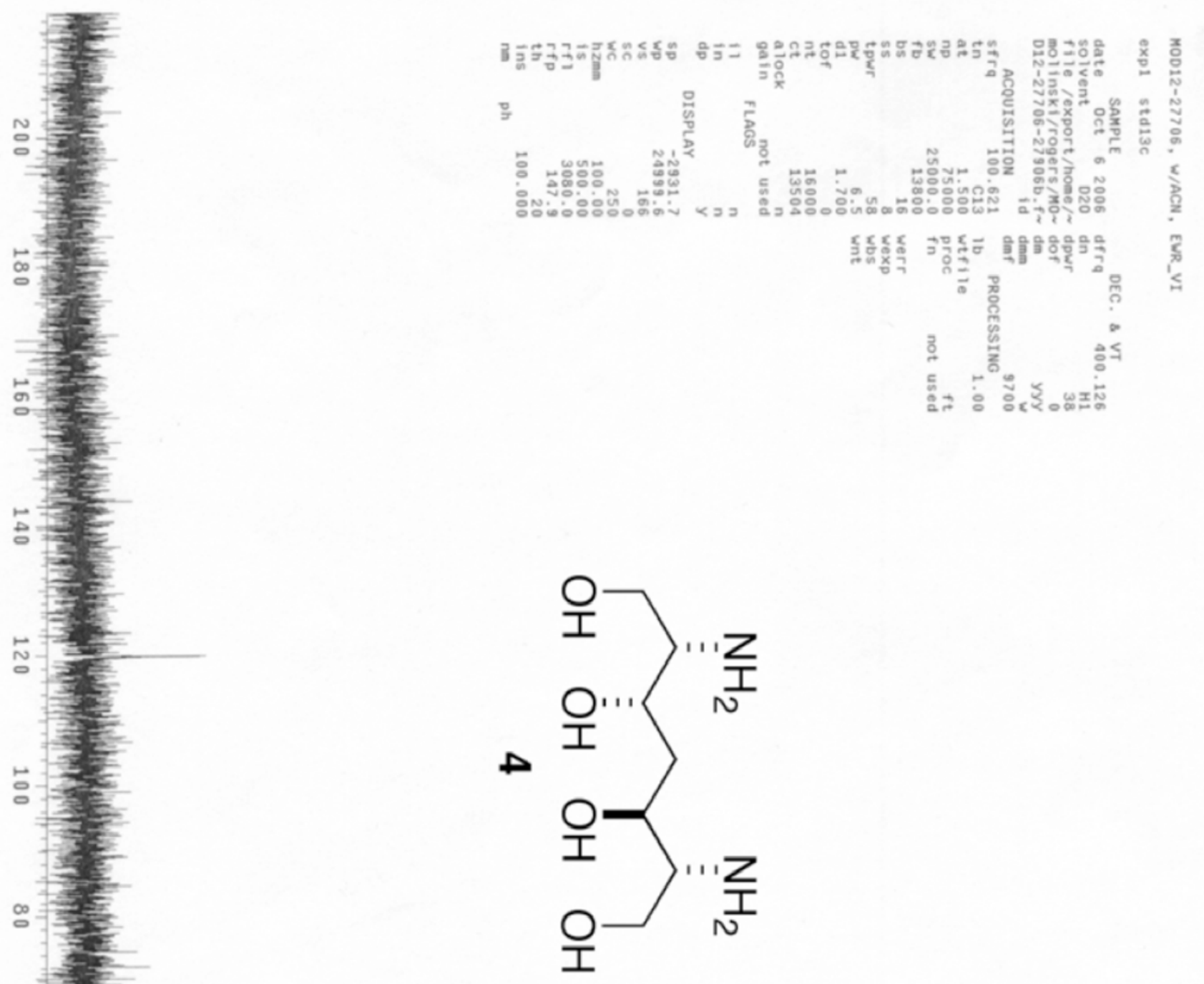




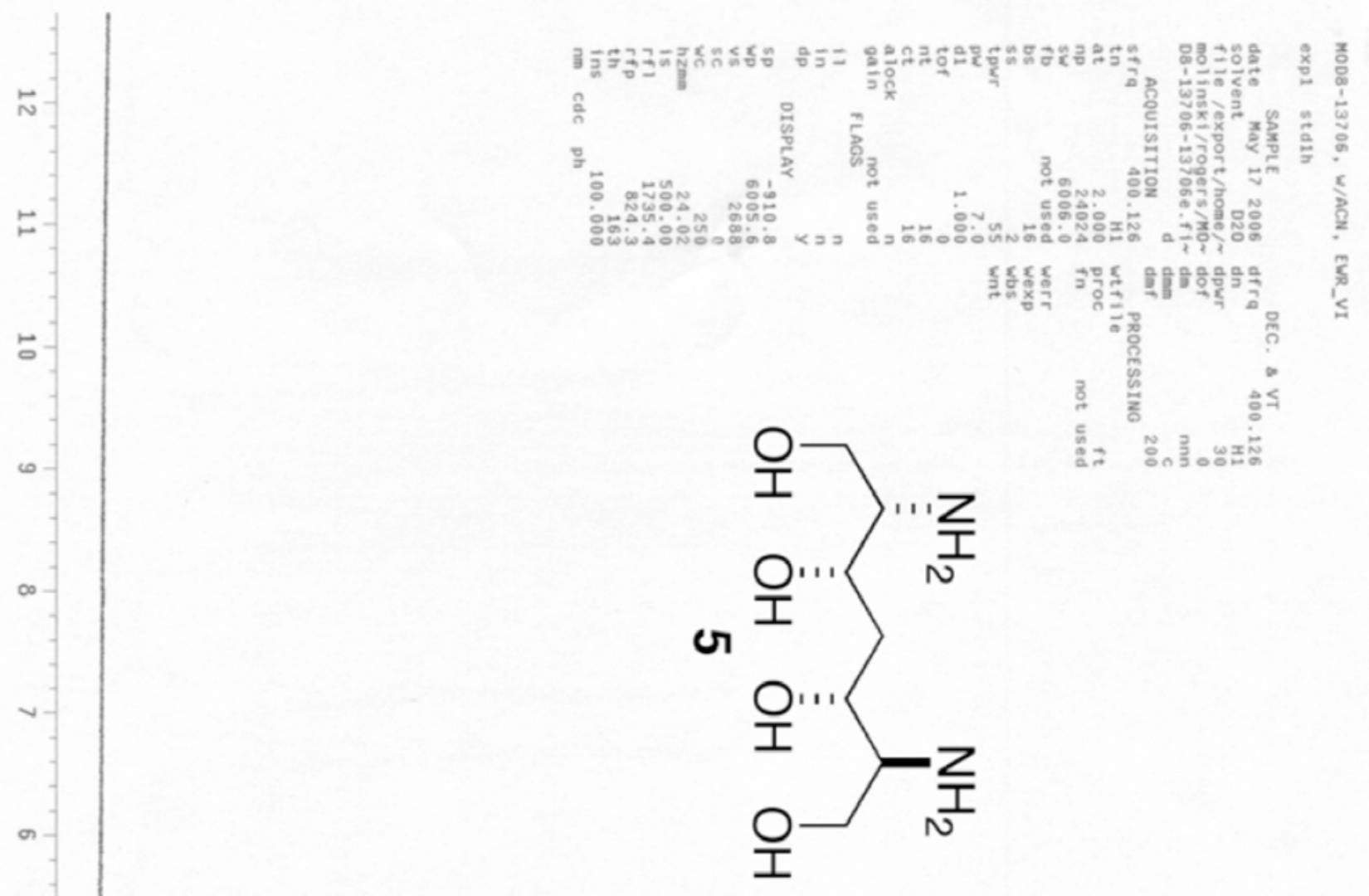




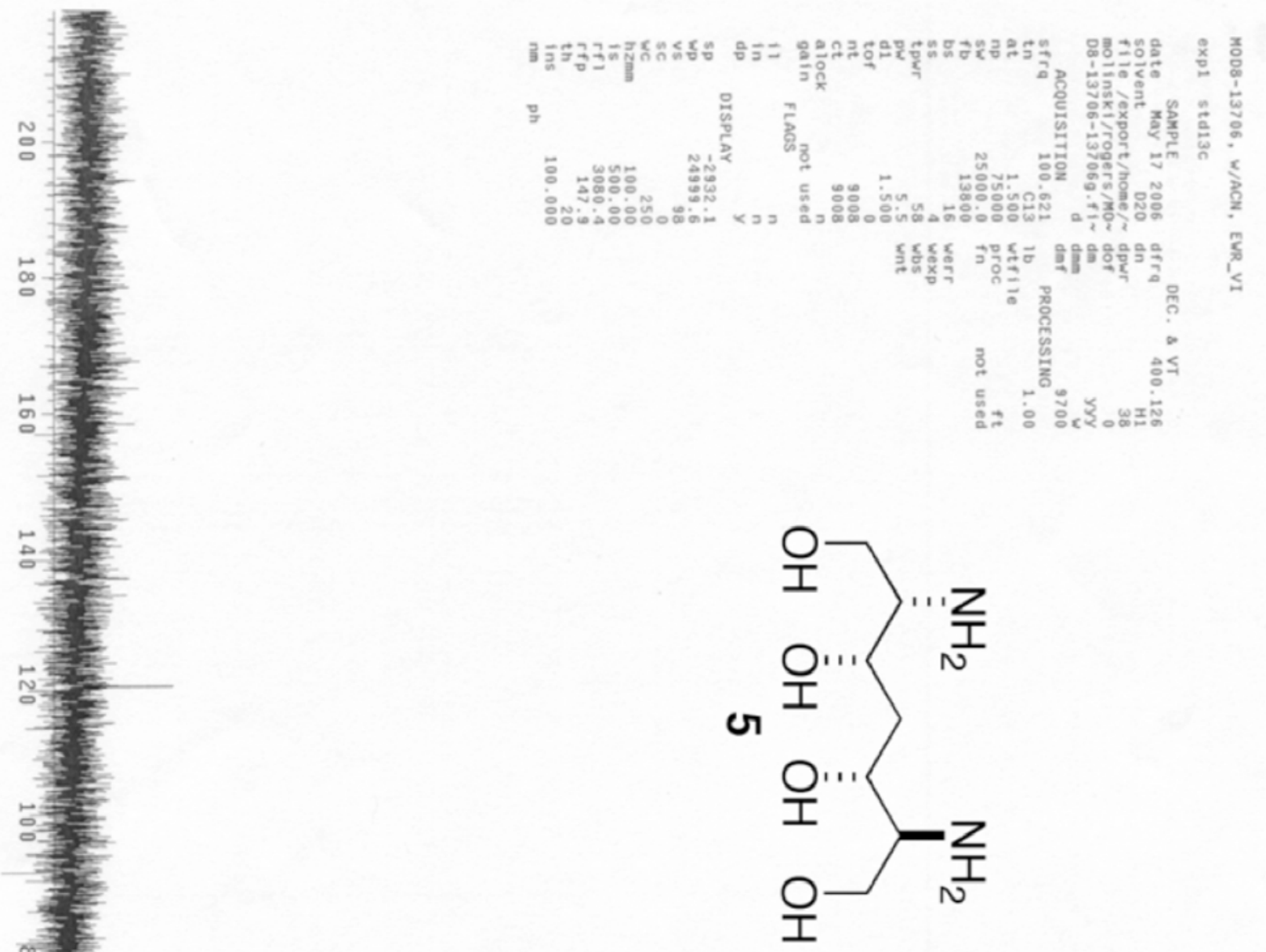




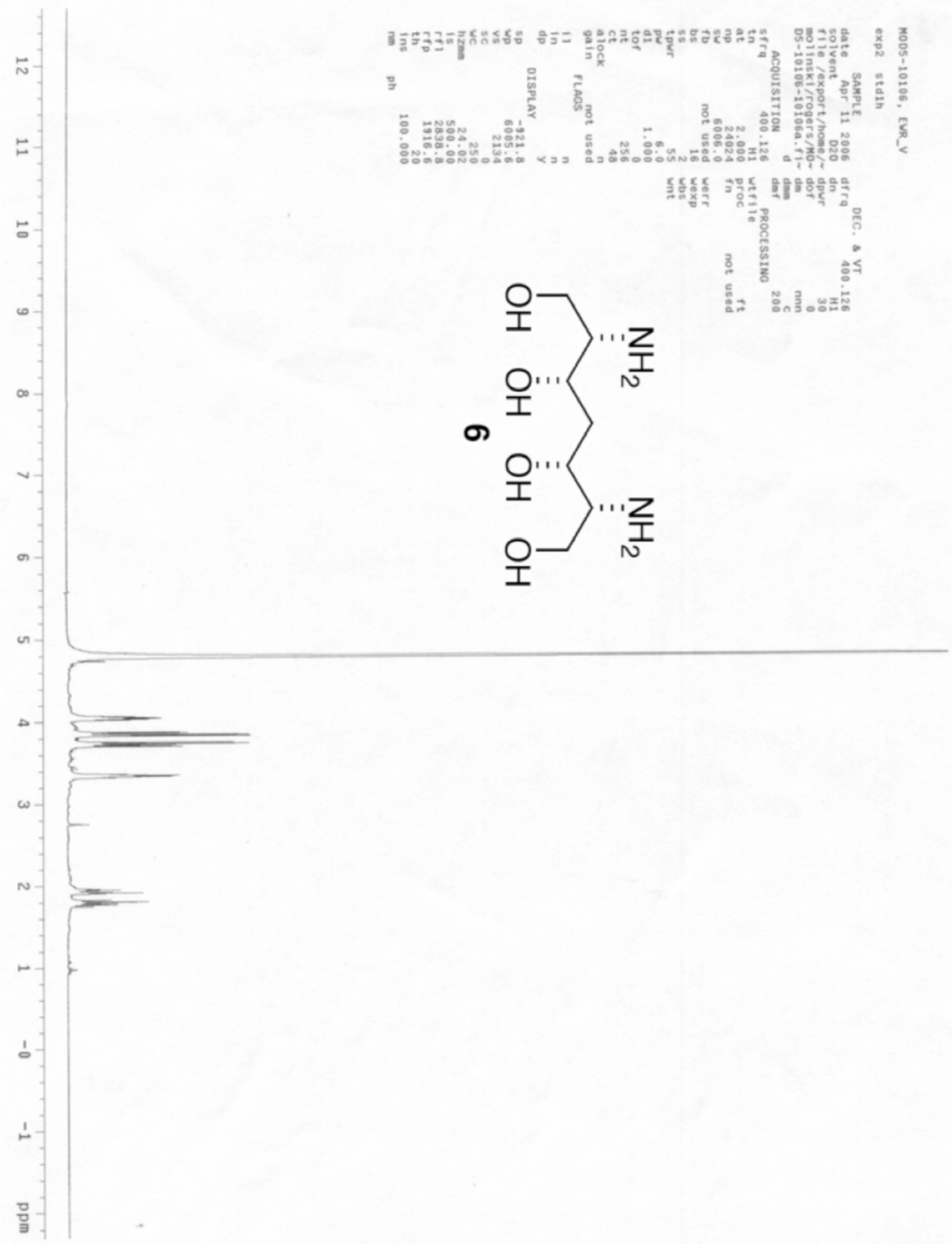




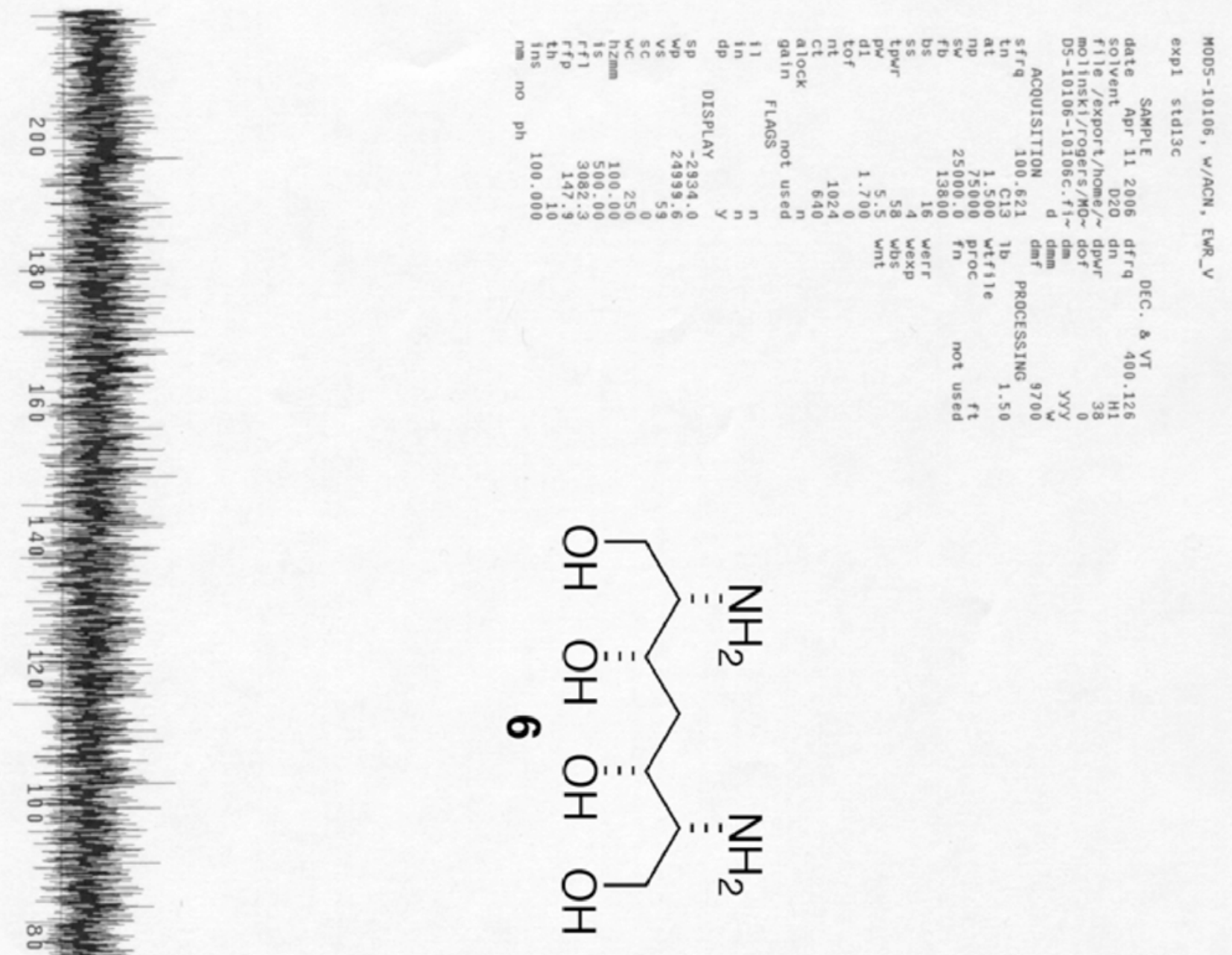




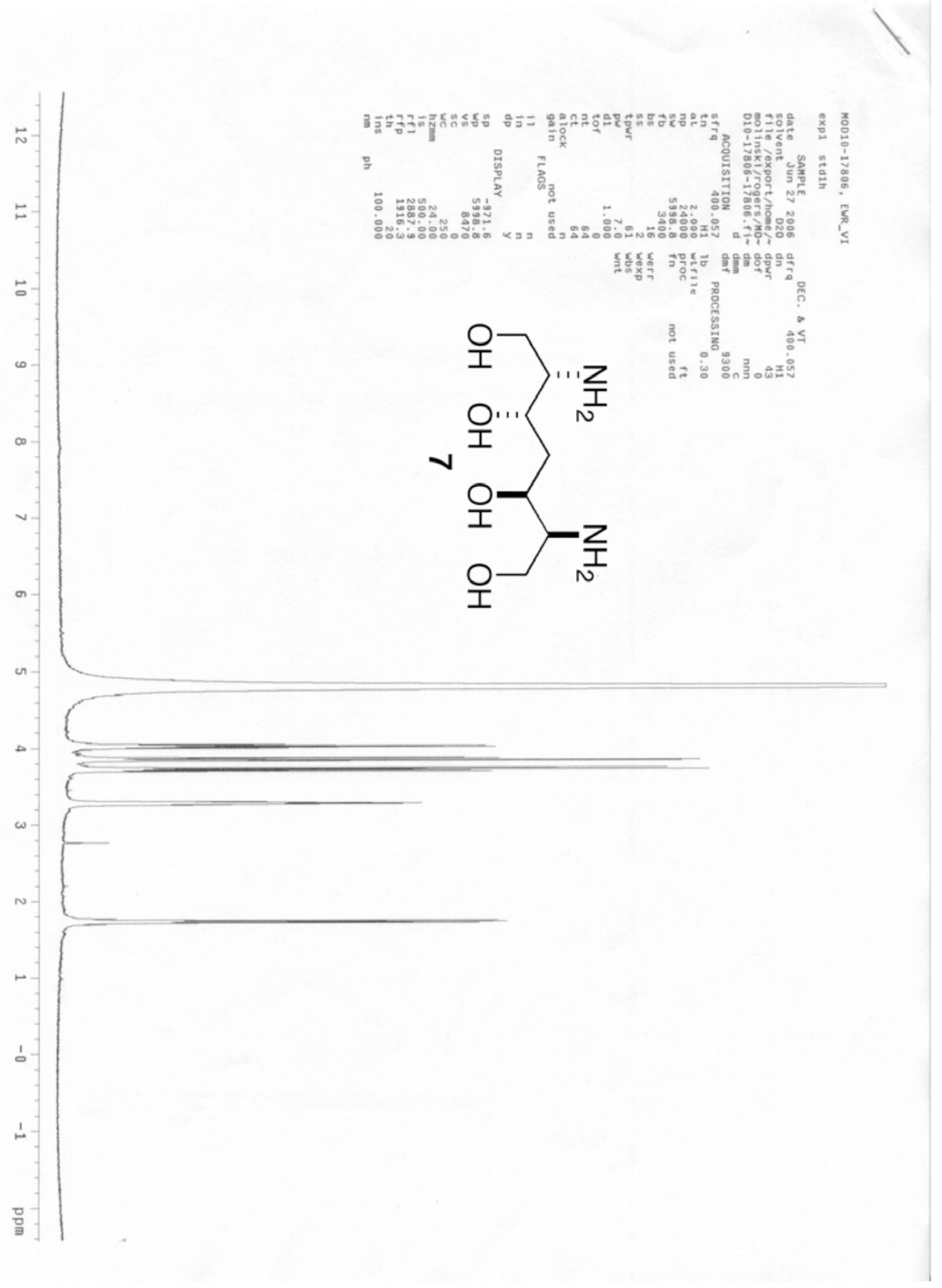




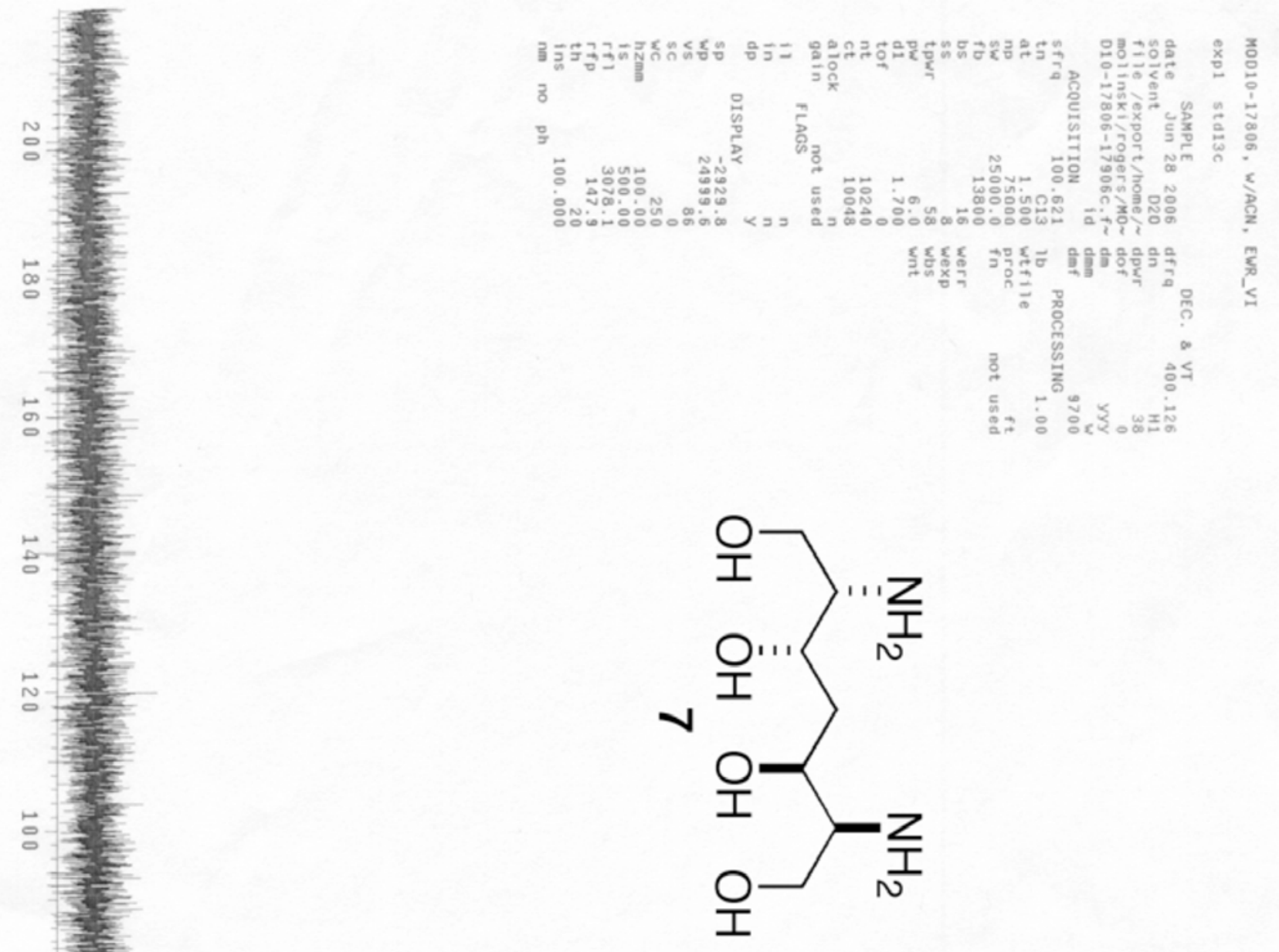




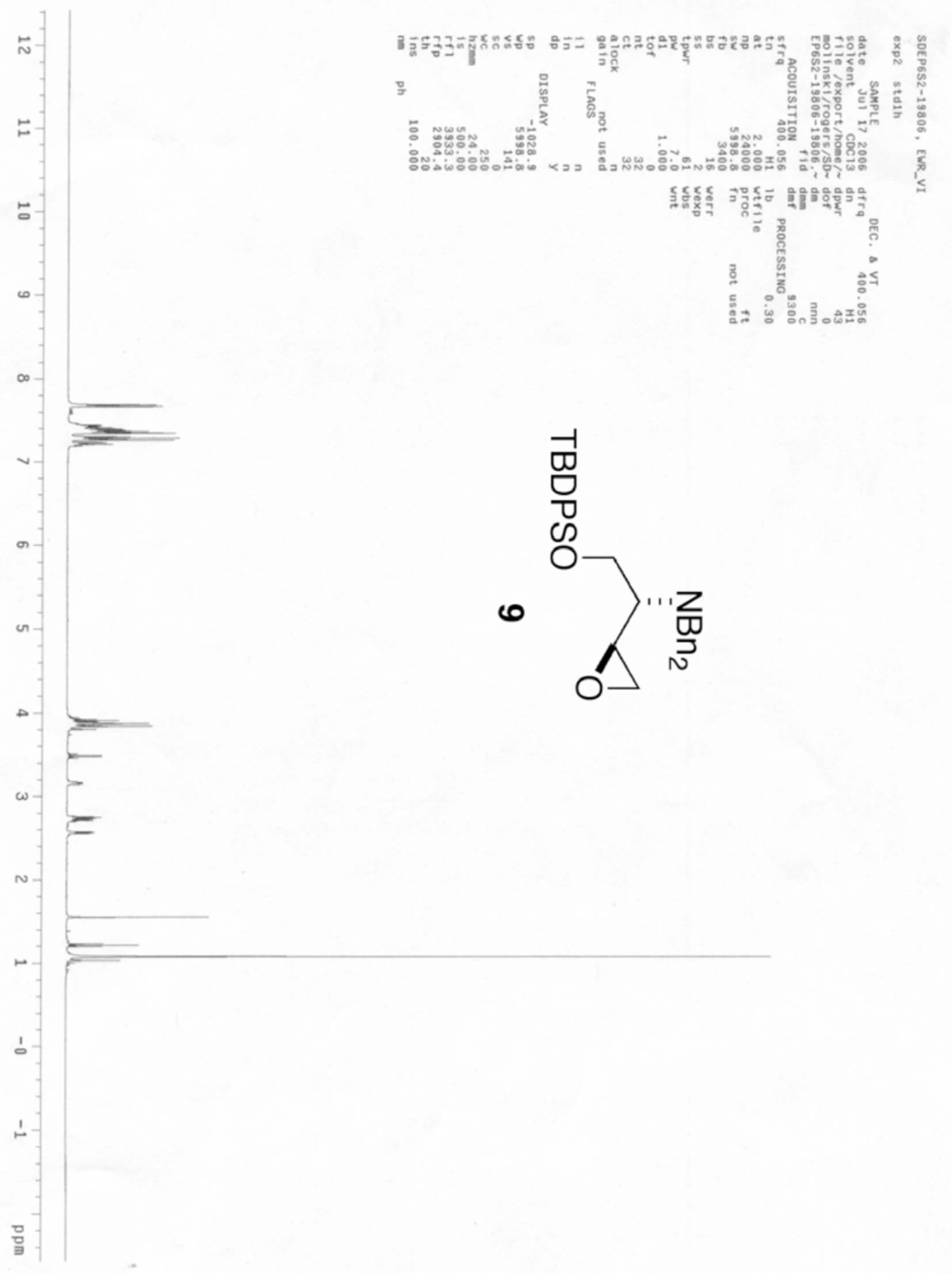




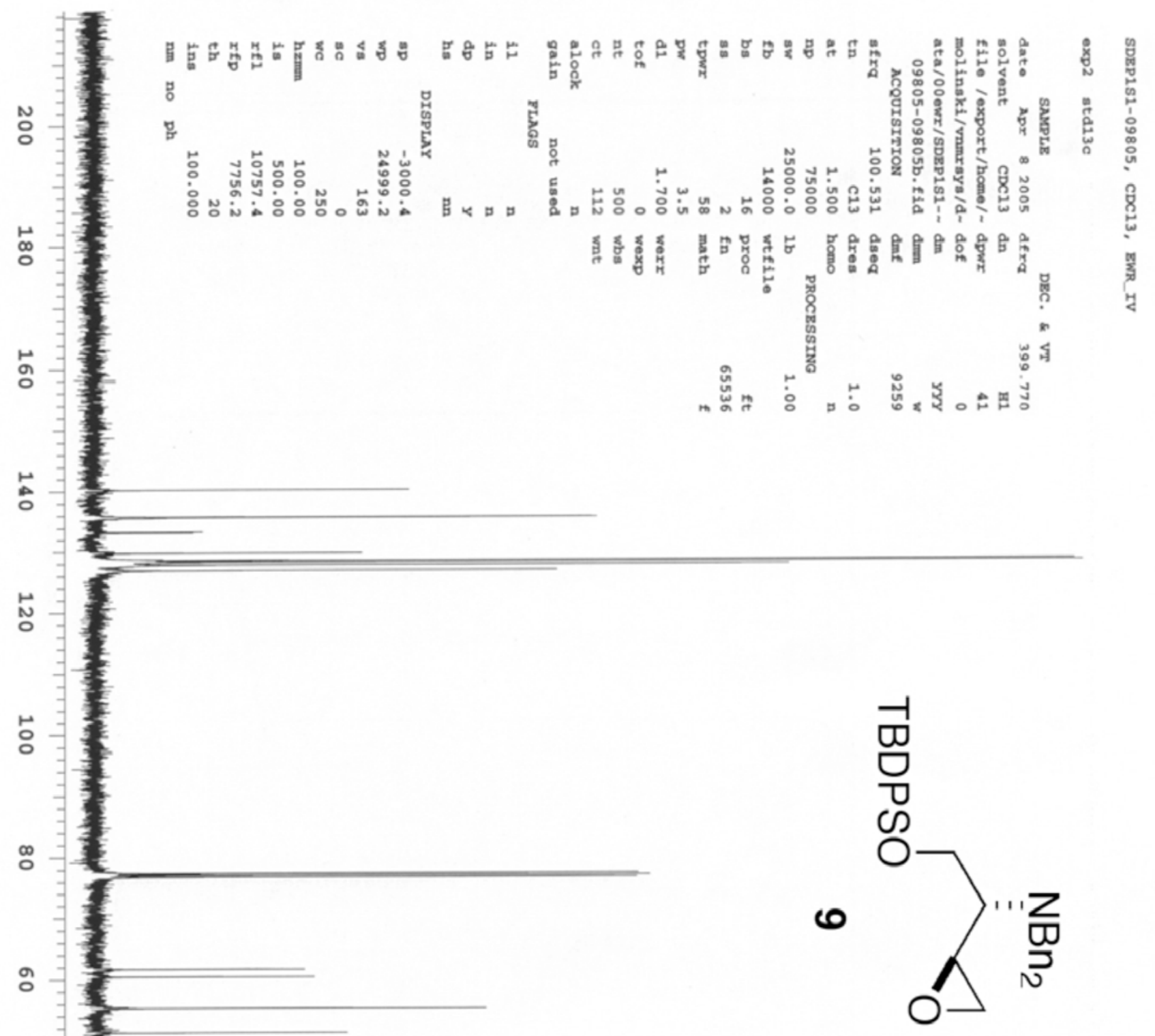




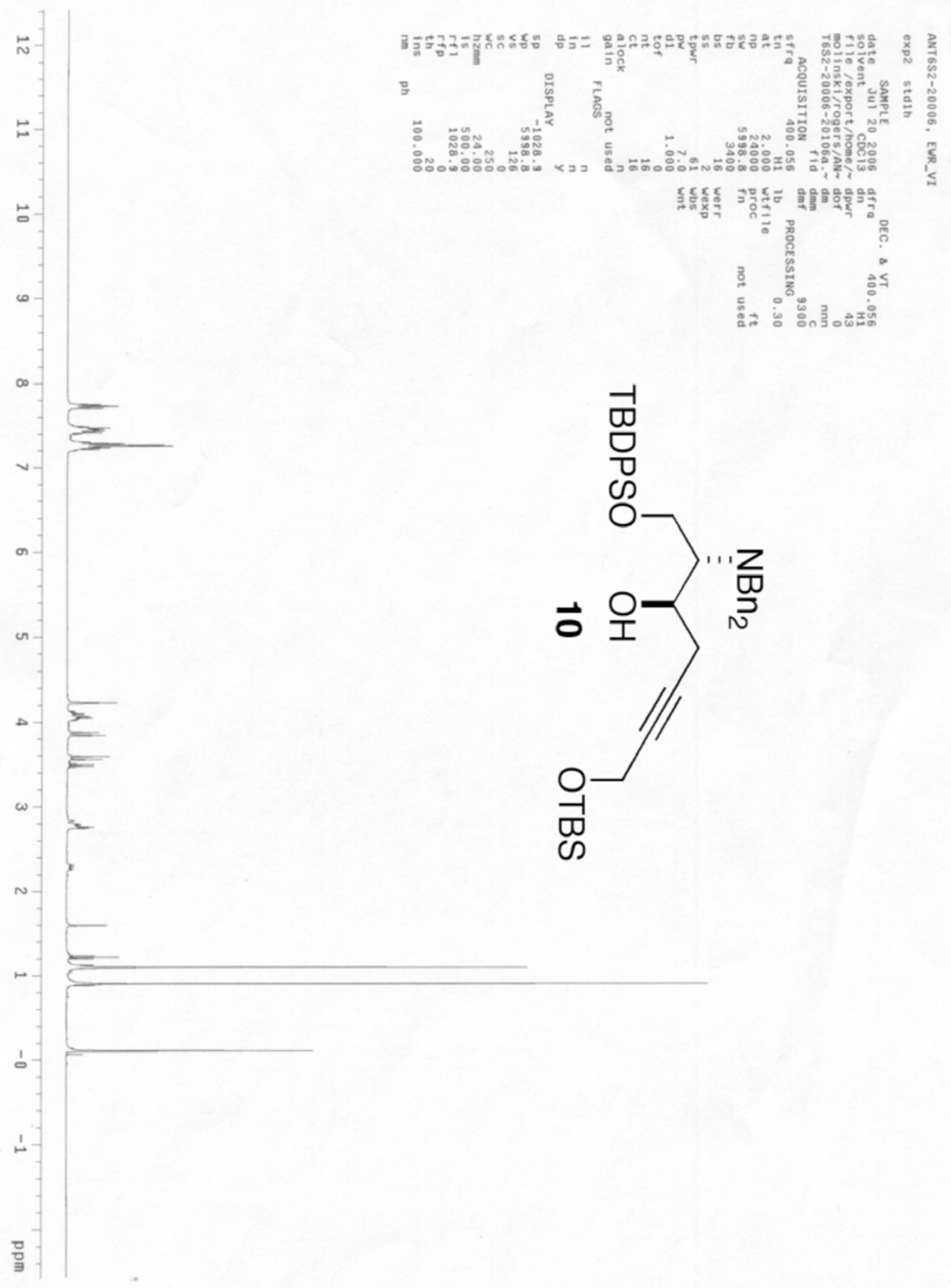




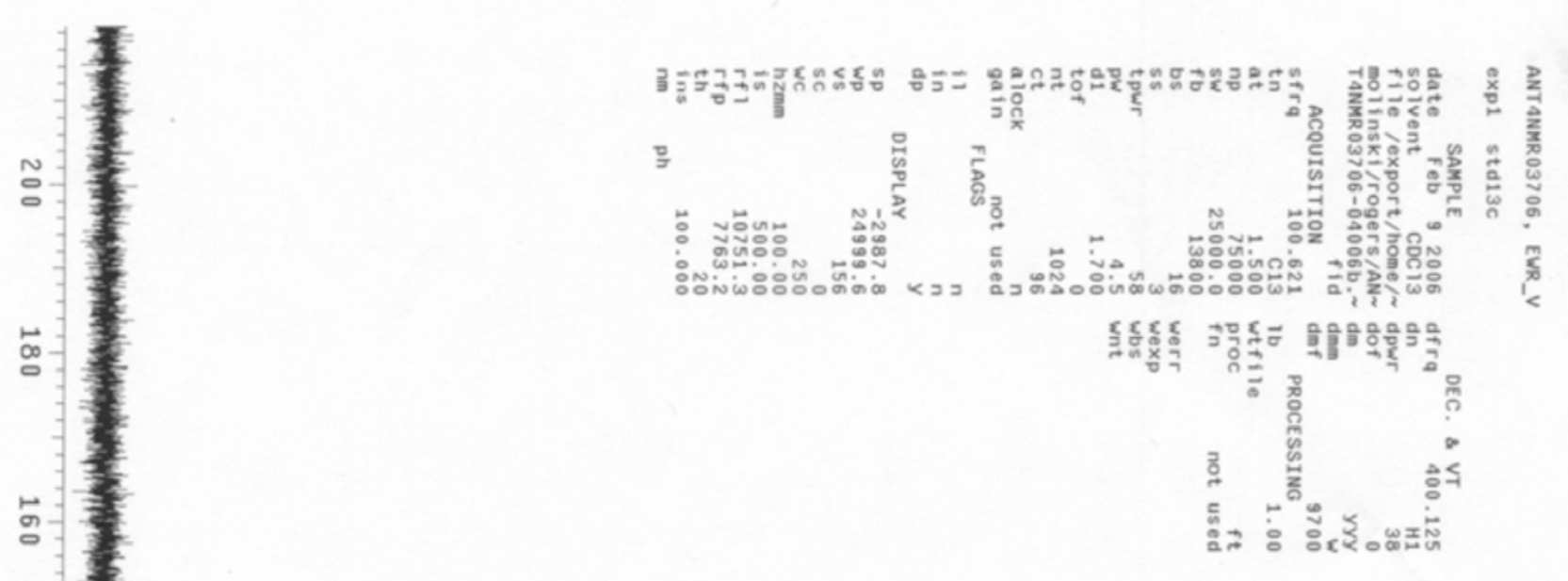

응

ㄴ.

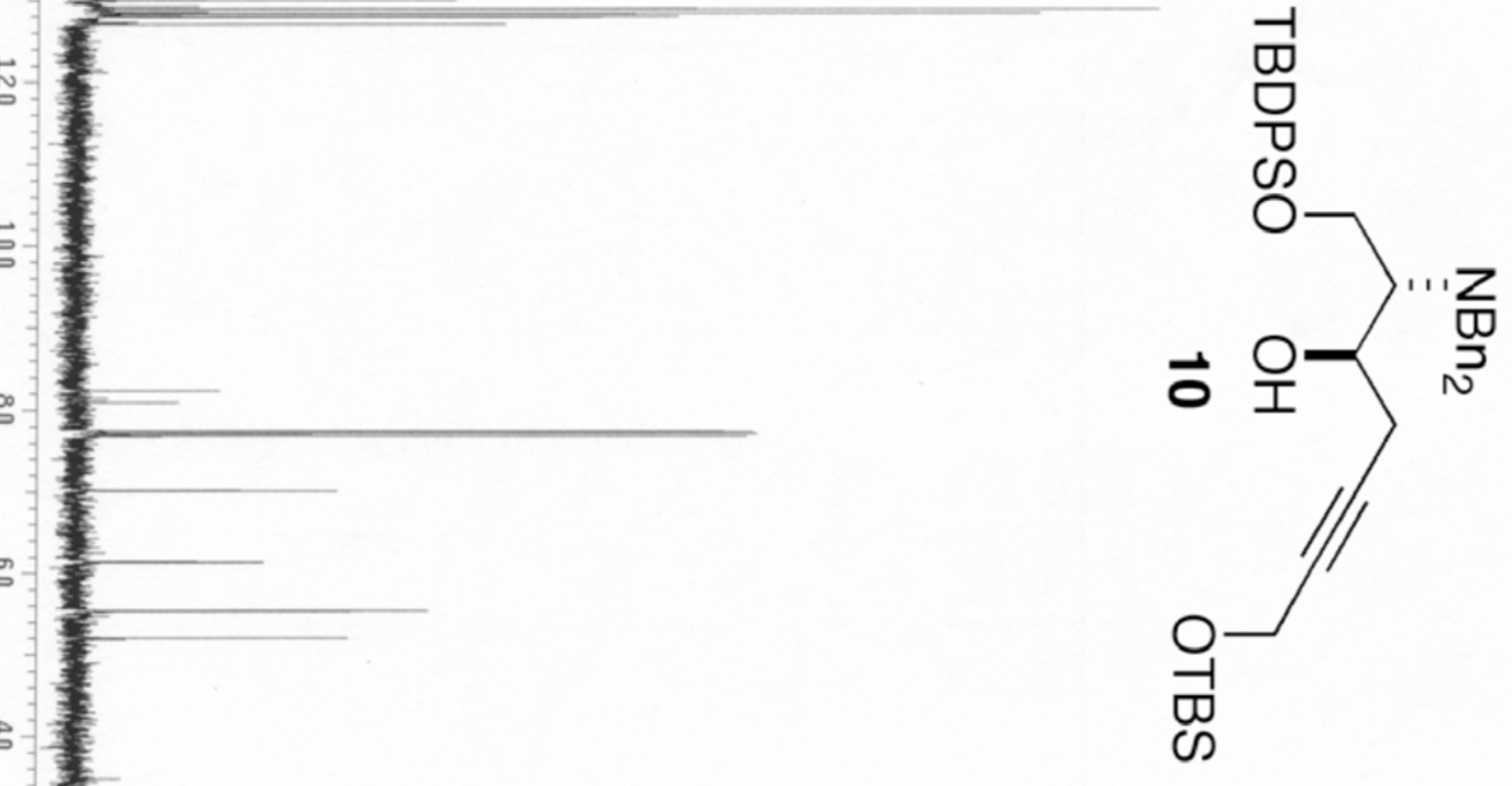

‥

0

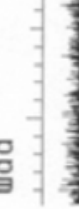




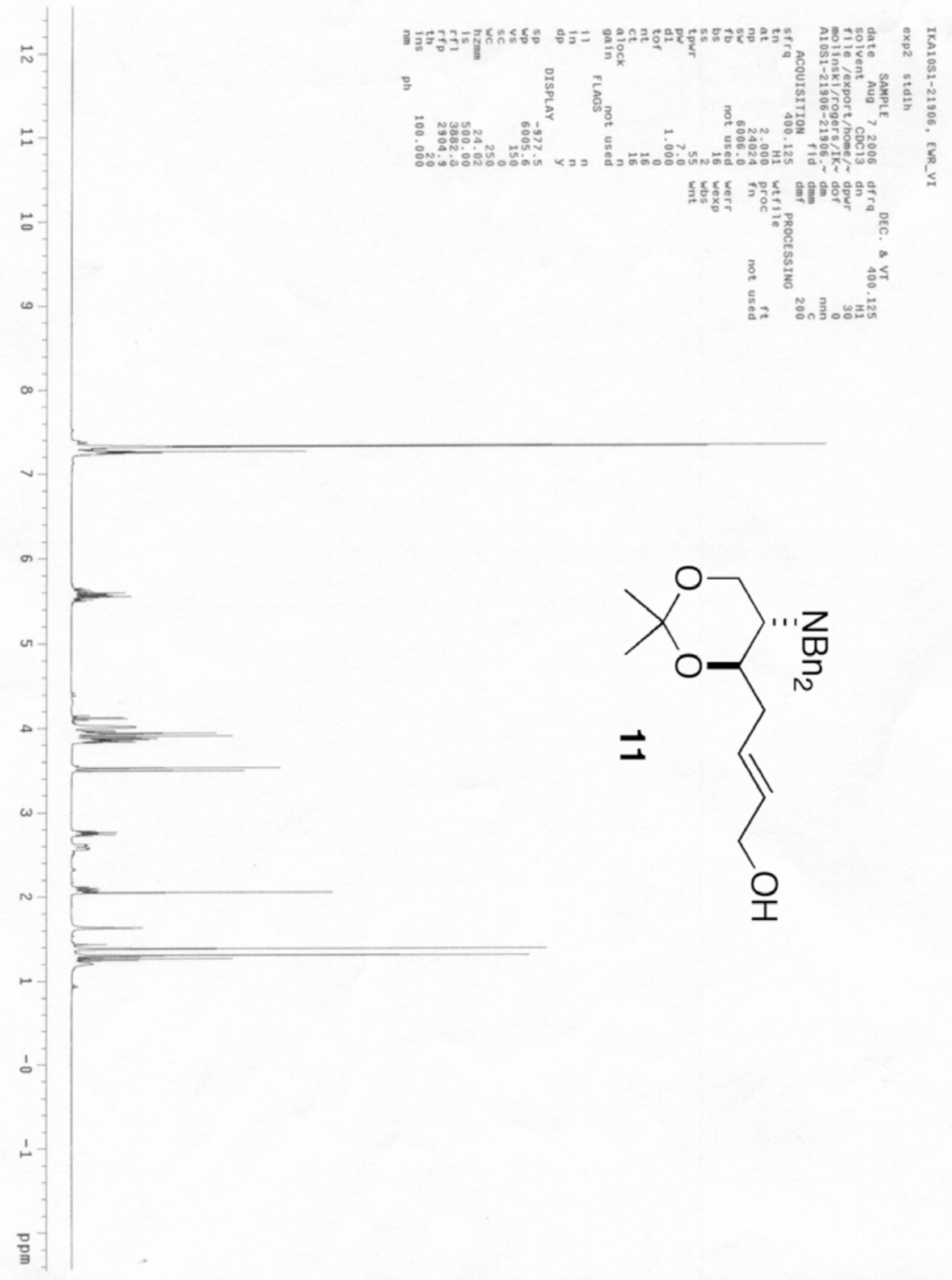




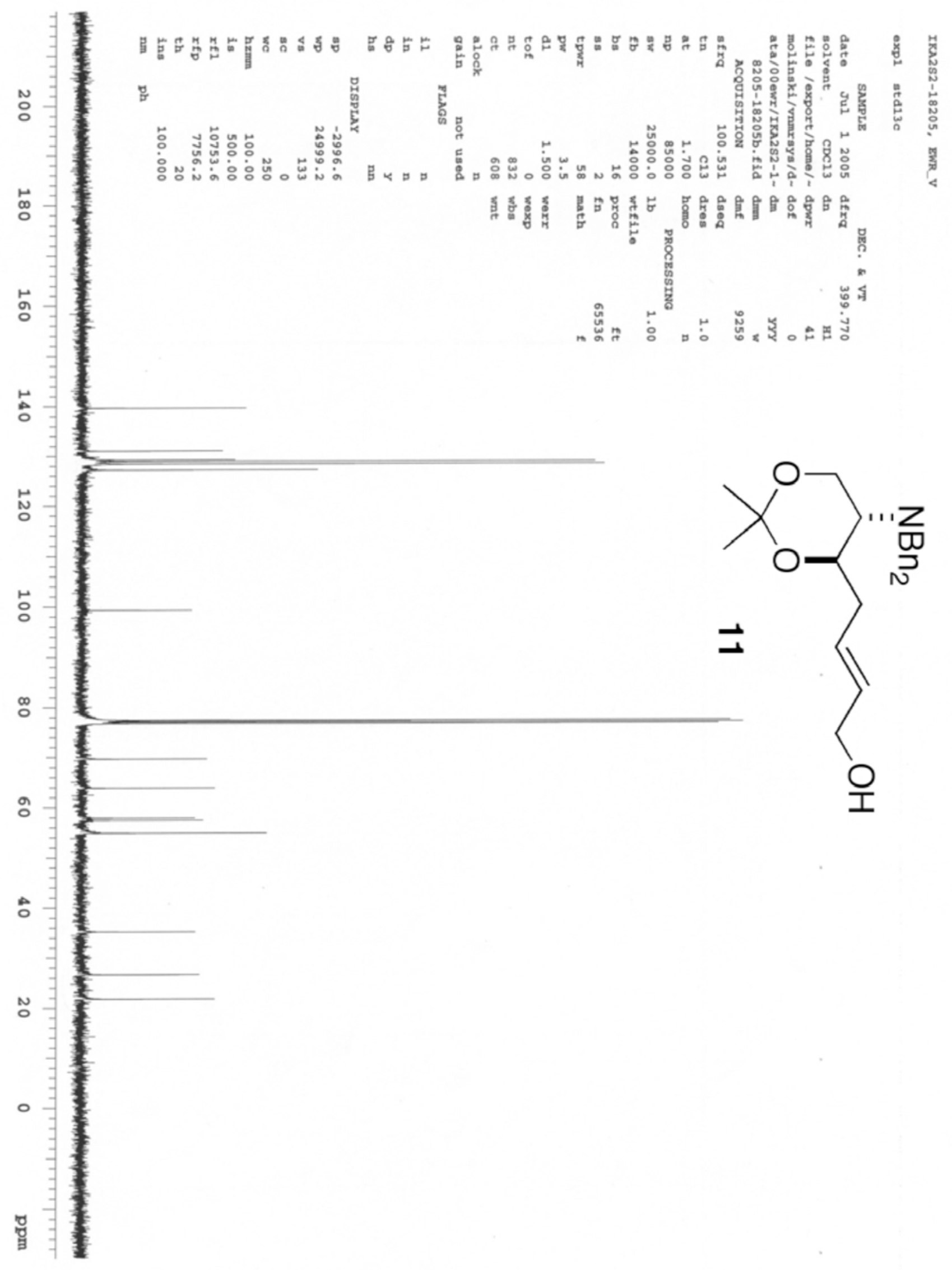



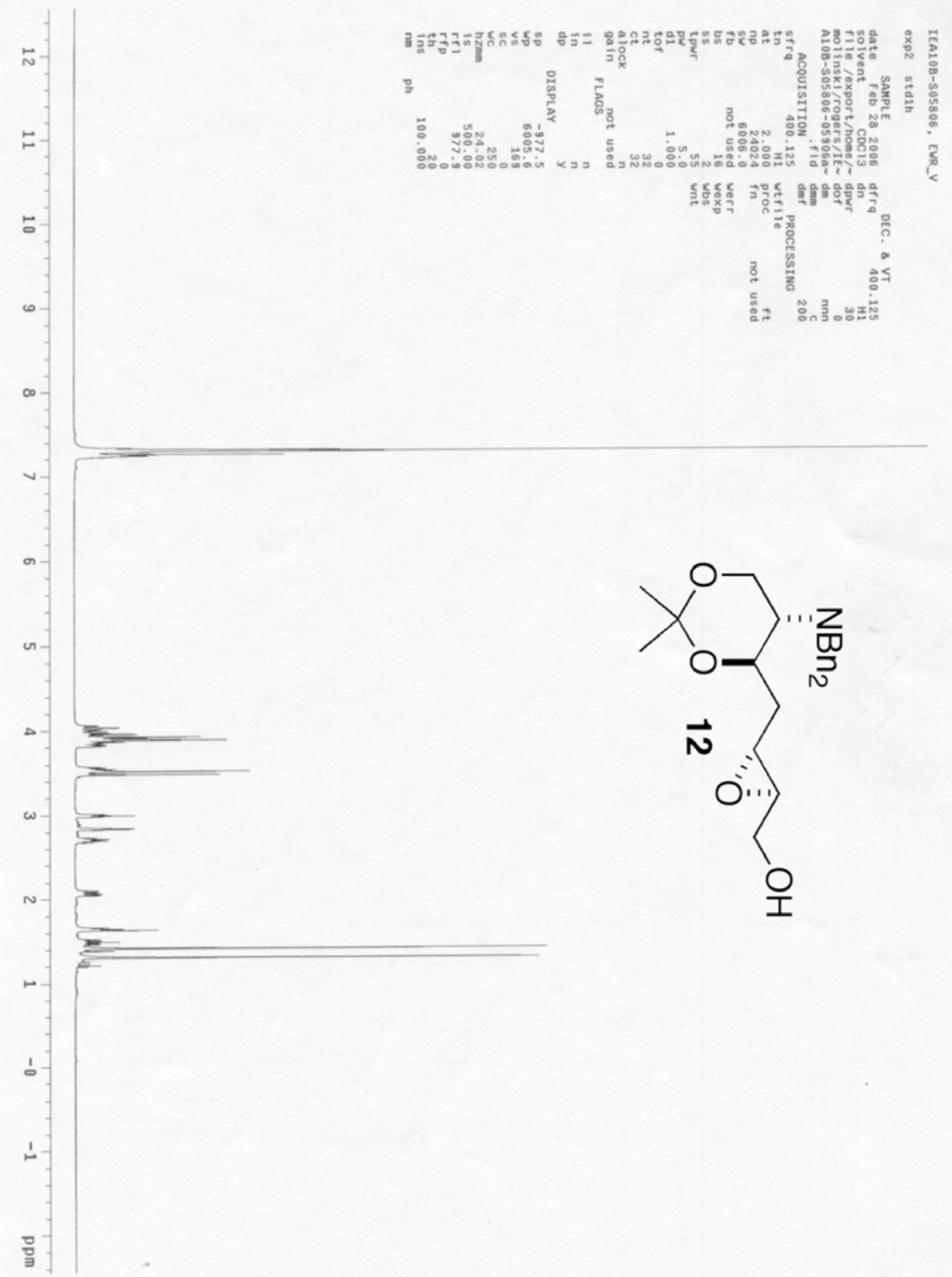

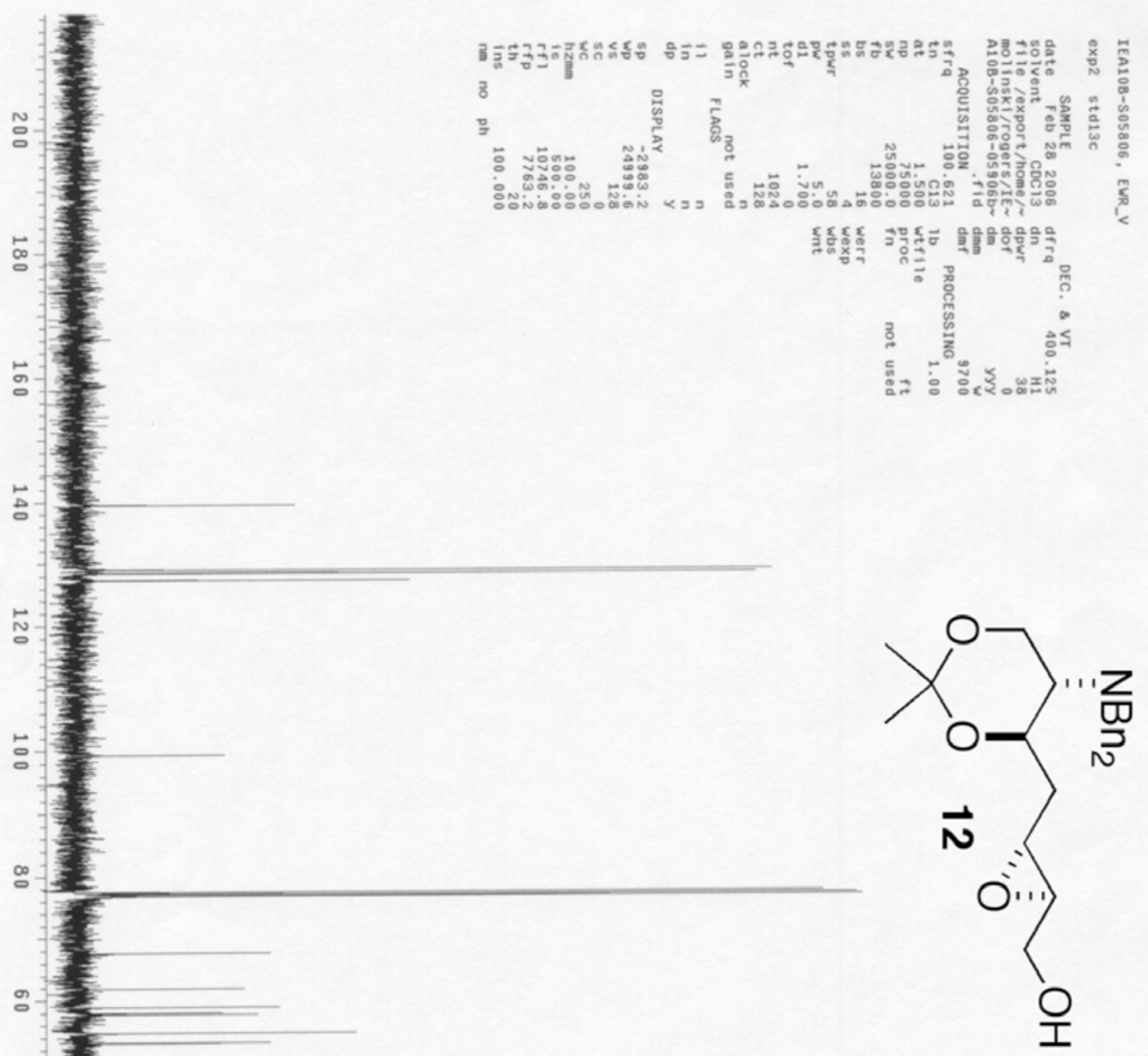


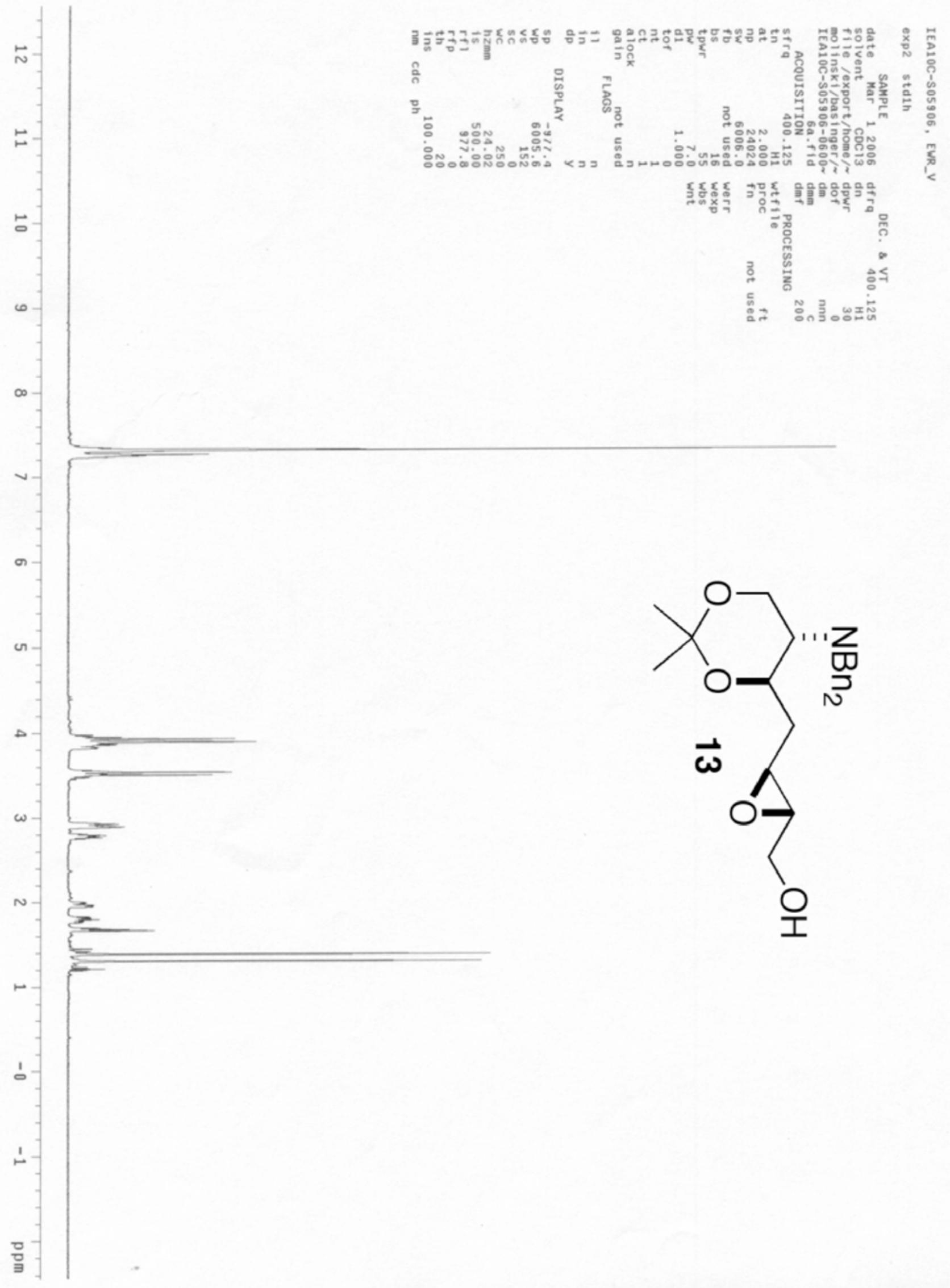




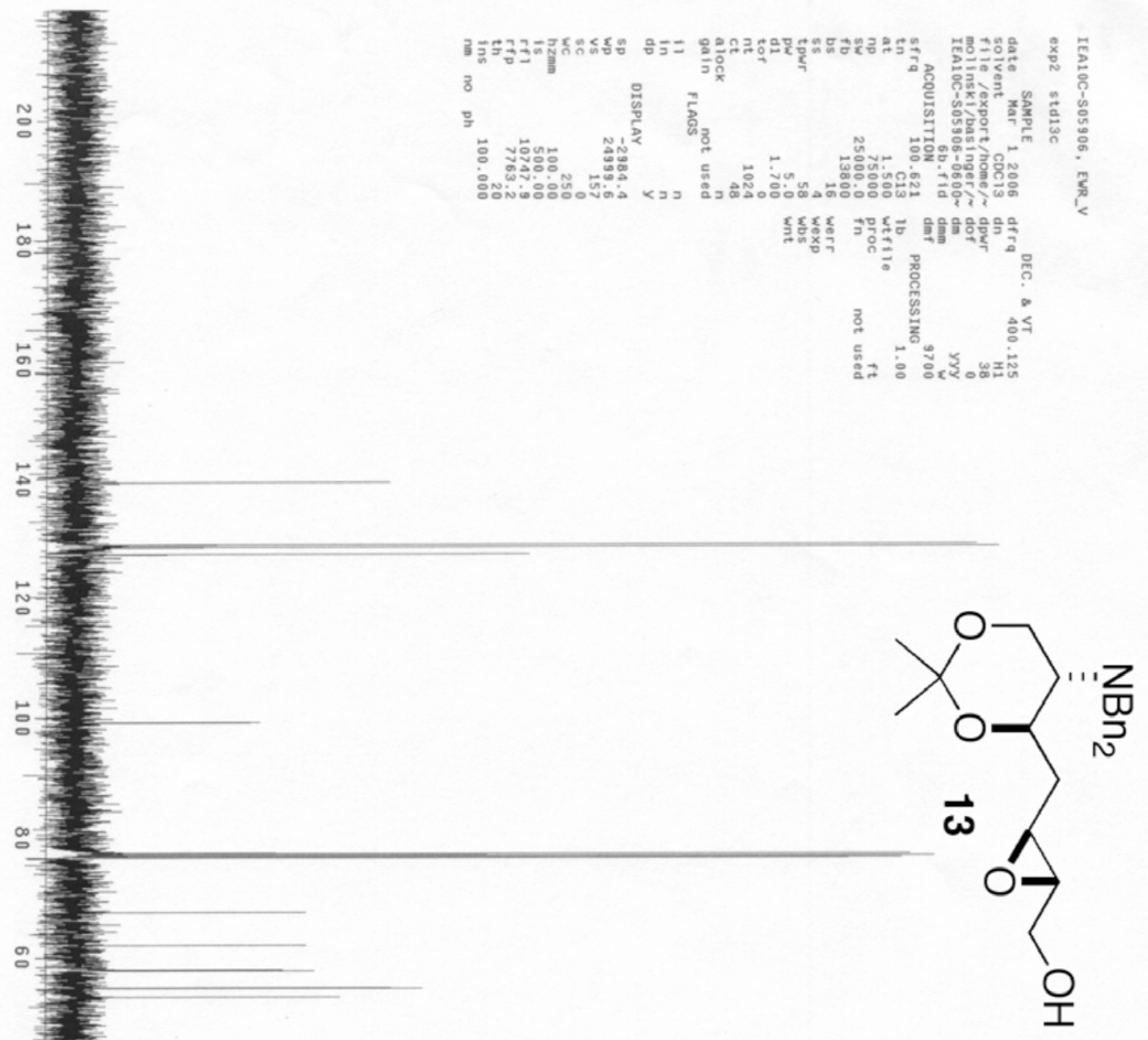



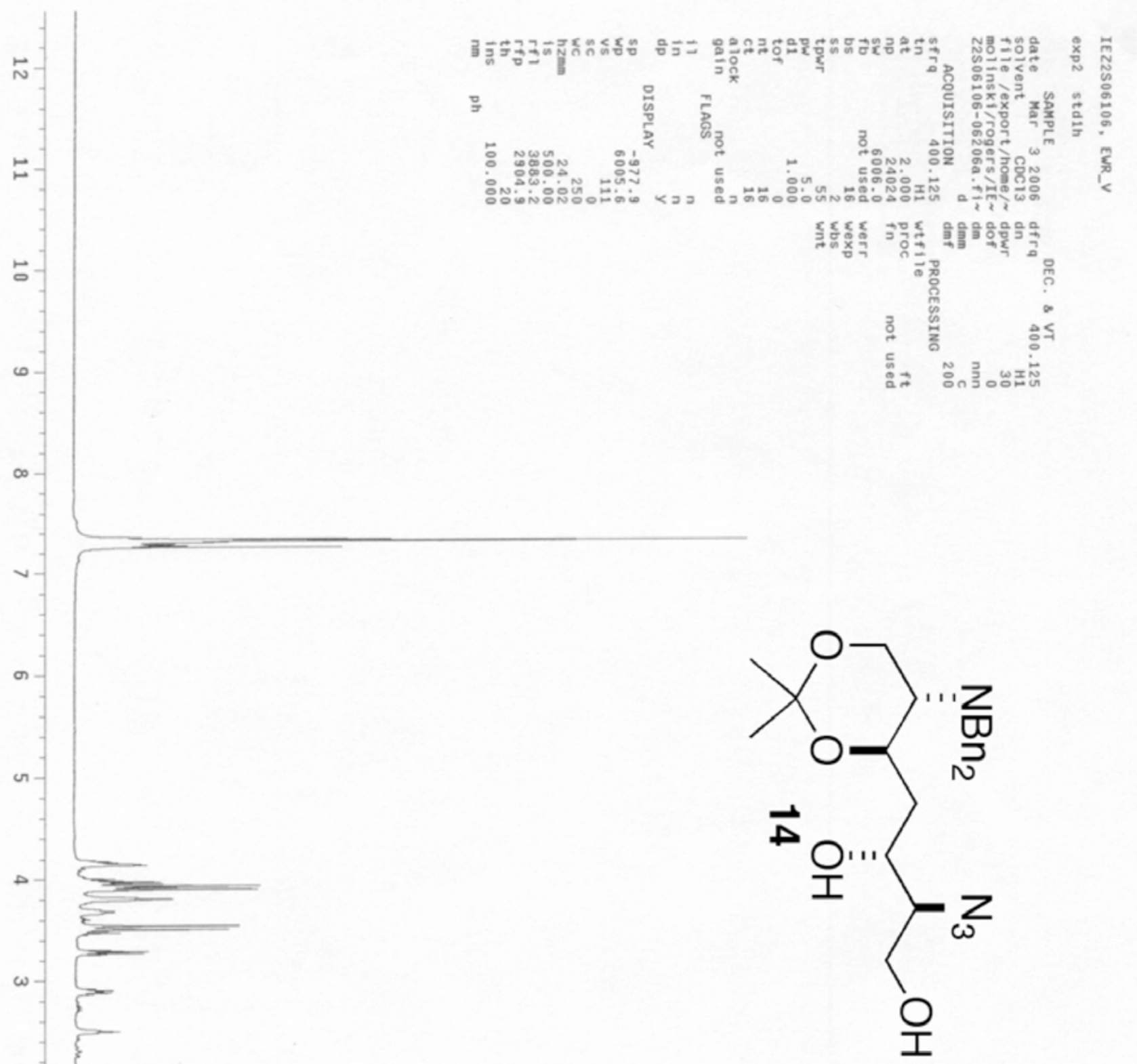

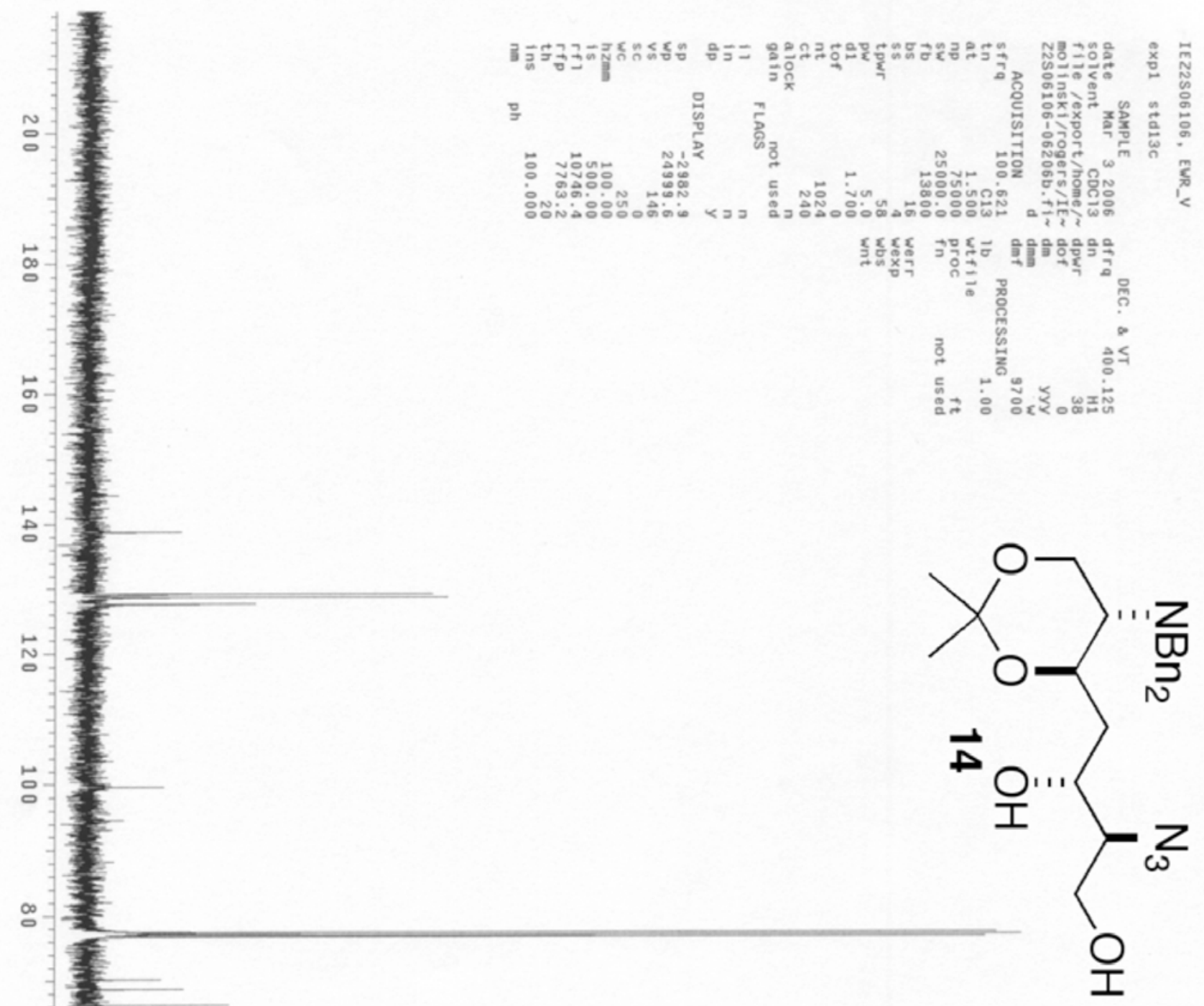

임

a

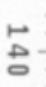

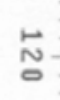

등

$\stackrel{\infty}{\circ}$

o-

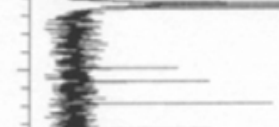

o-

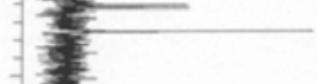

N.

-

$\circ$

持帘

㝵衰 


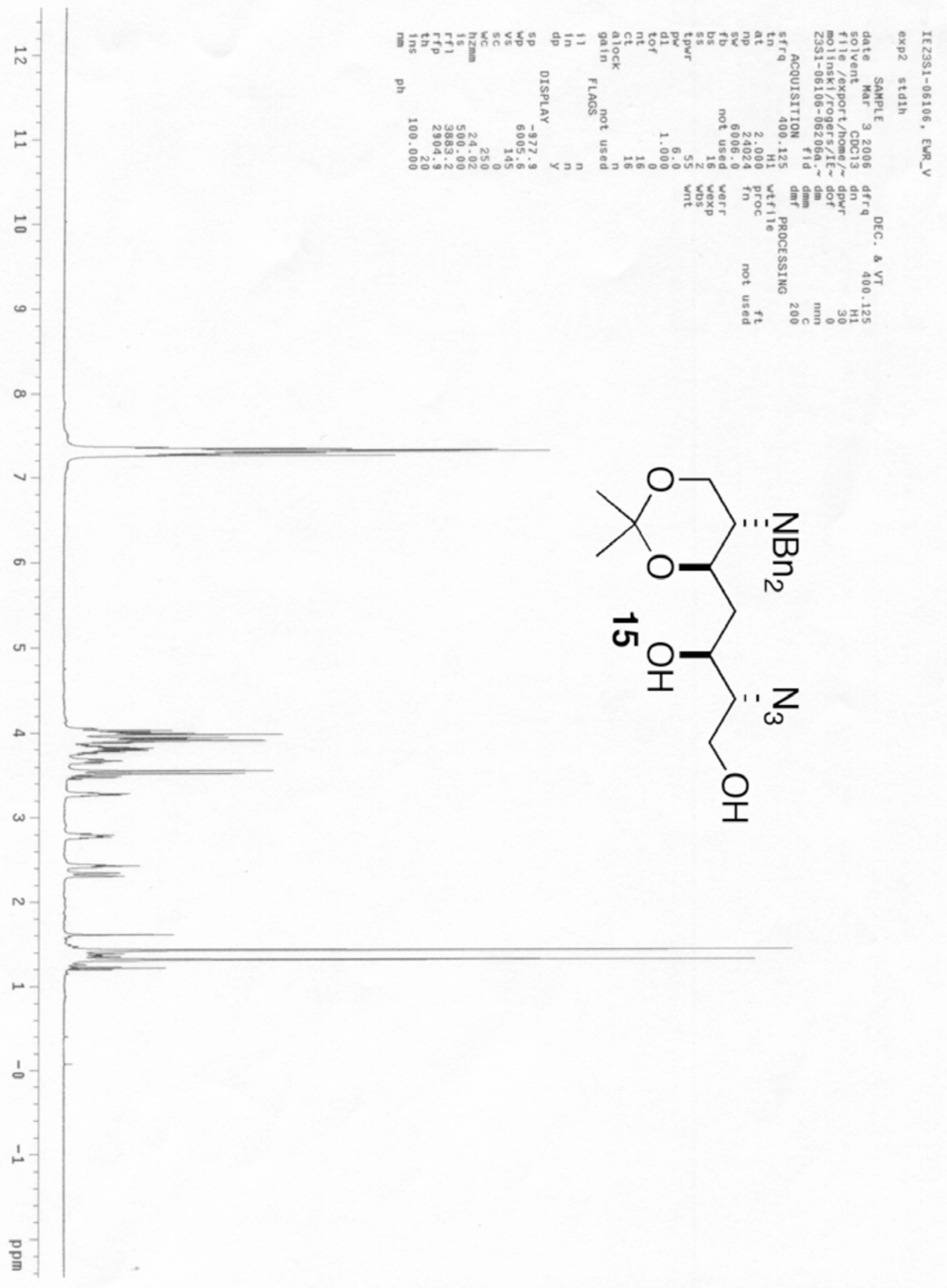




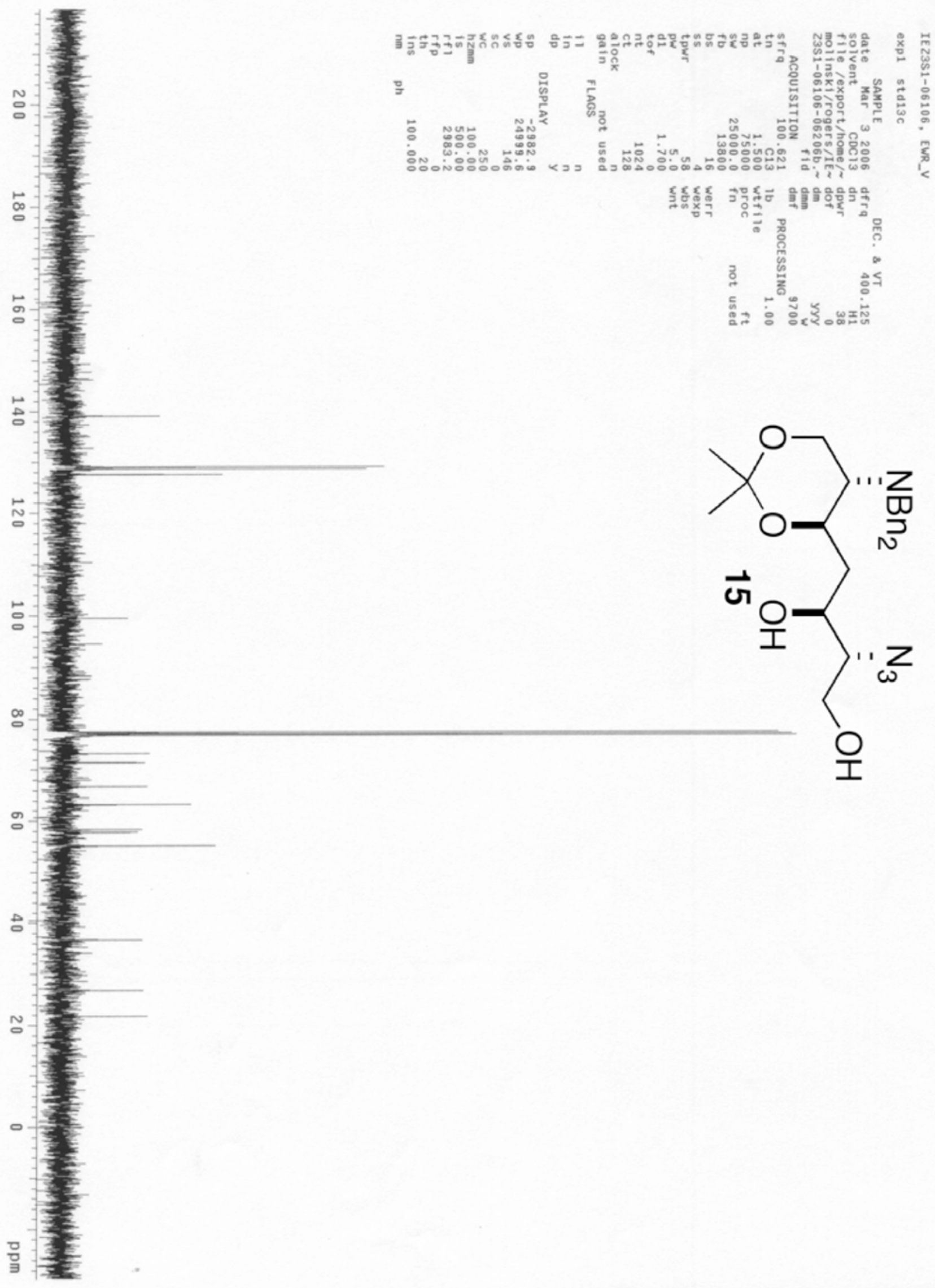




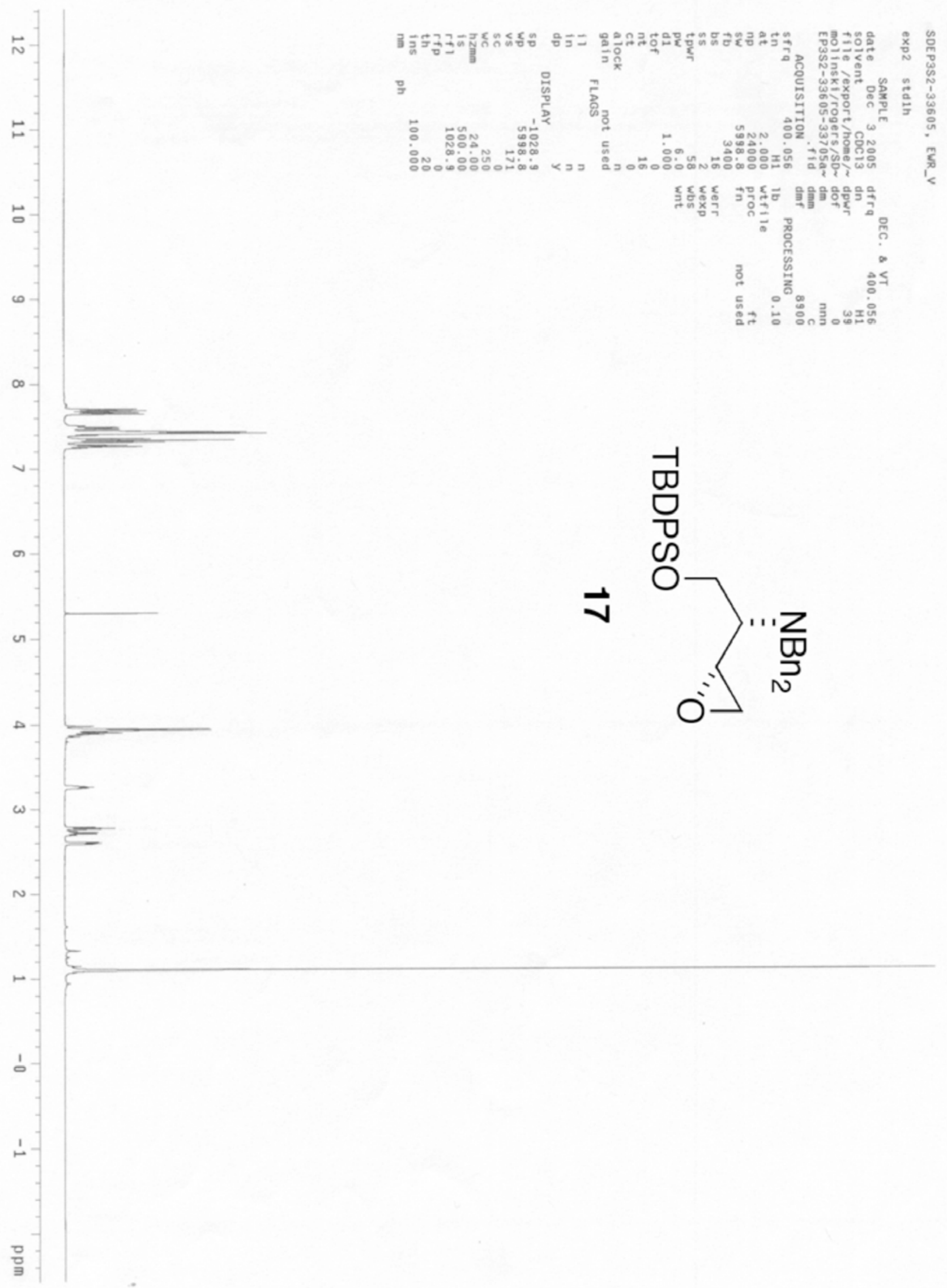




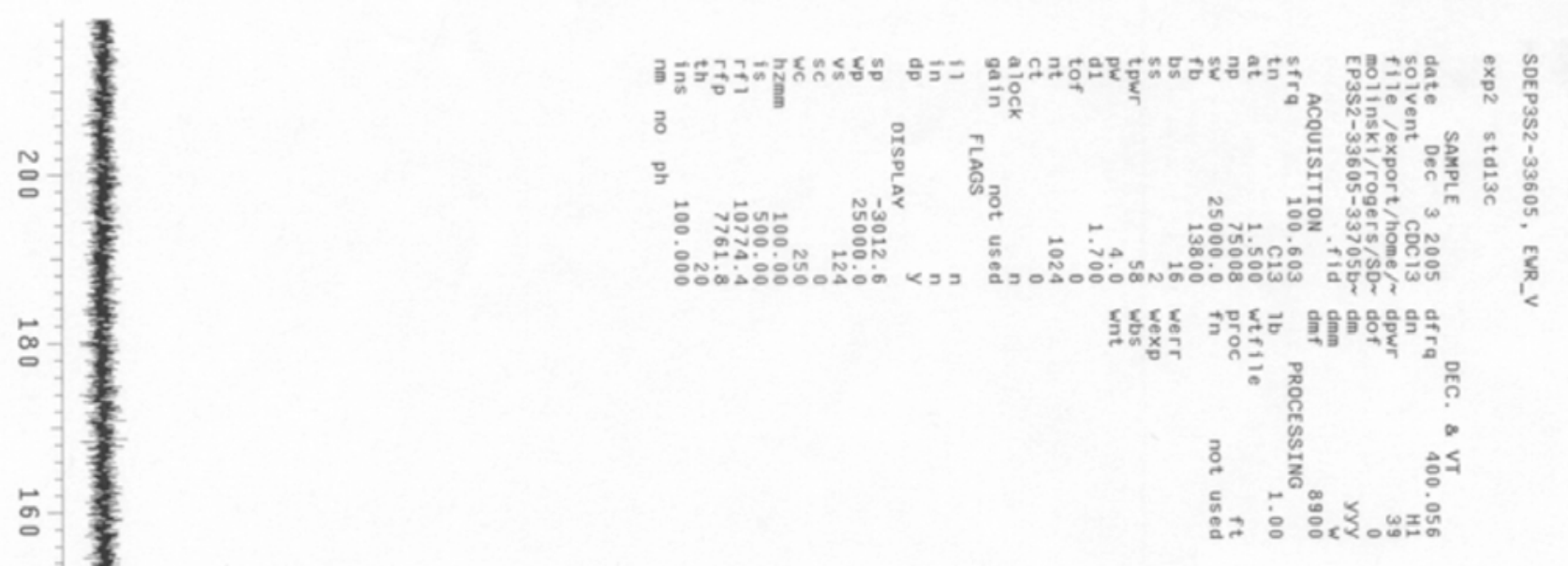

点
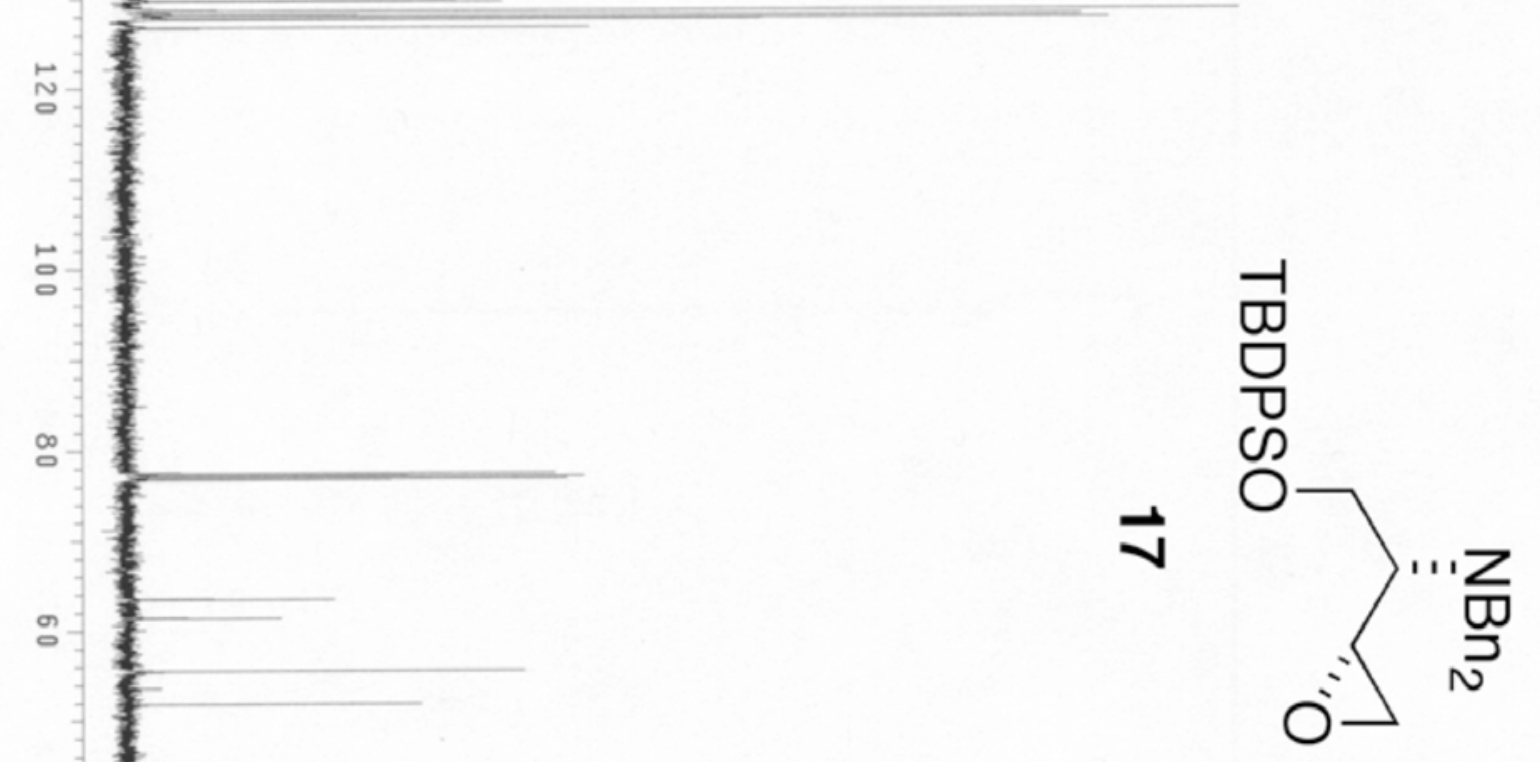

。

응

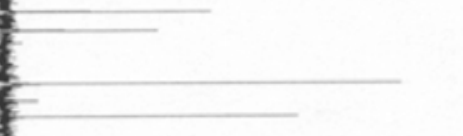

$\stackrel{\circ}{\circ}$

$\stackrel{\infty}{\circ}$

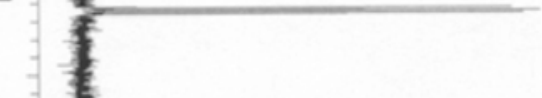

N

3
3
3
3
3
3
3 


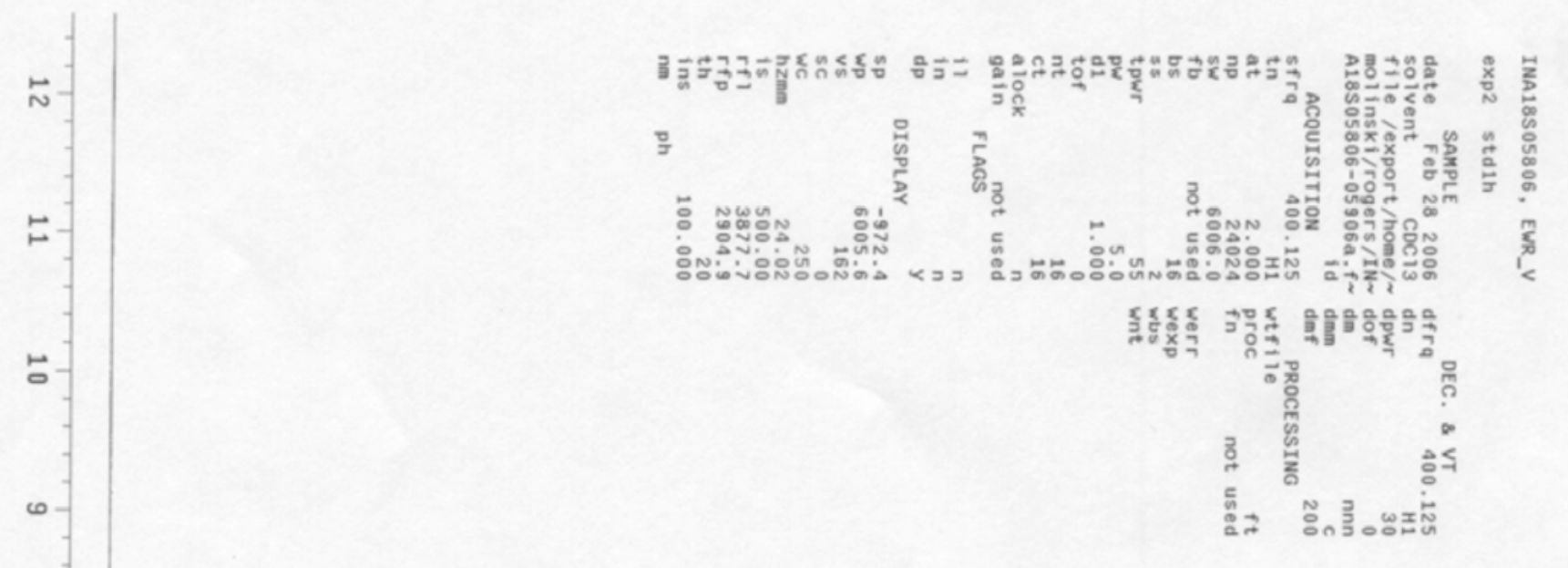

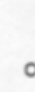
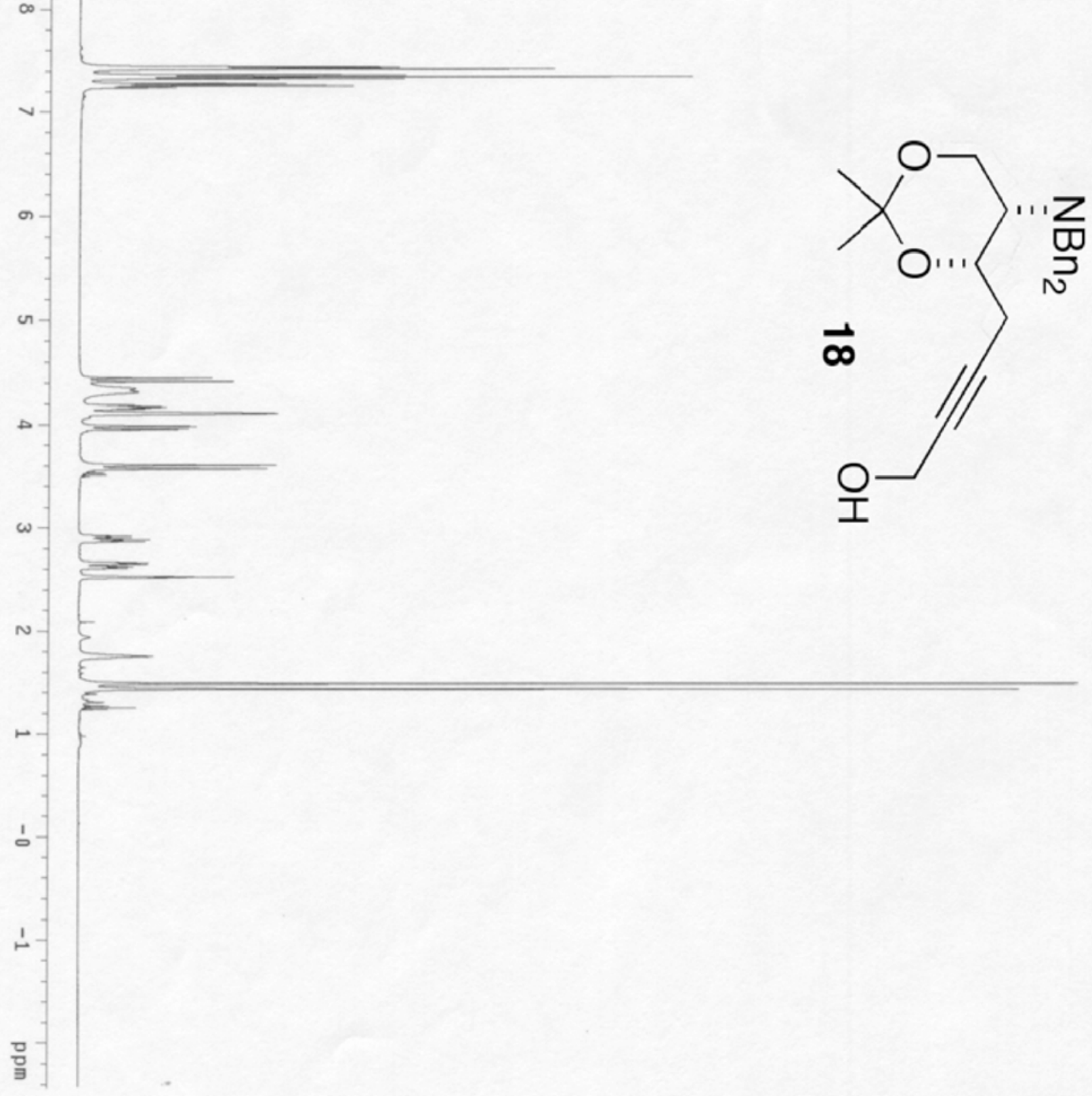


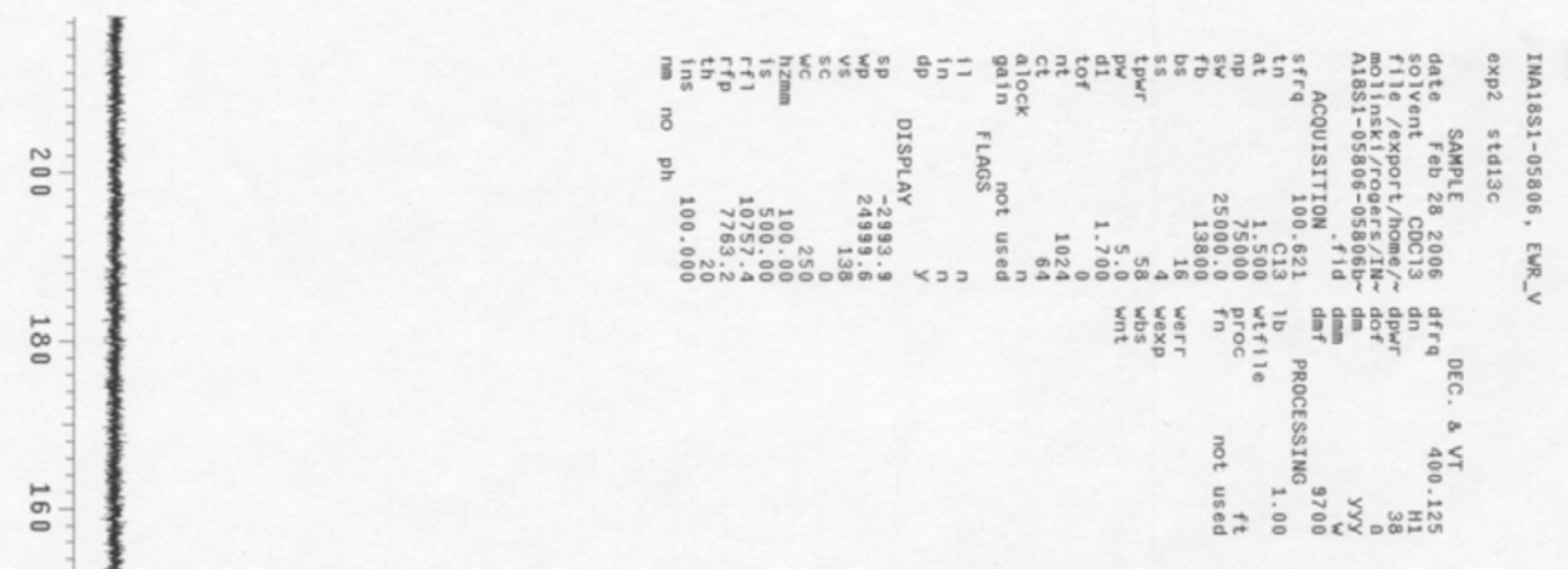

$\stackrel{\circ}{\circ}$

$\stackrel{5}{0}$

응

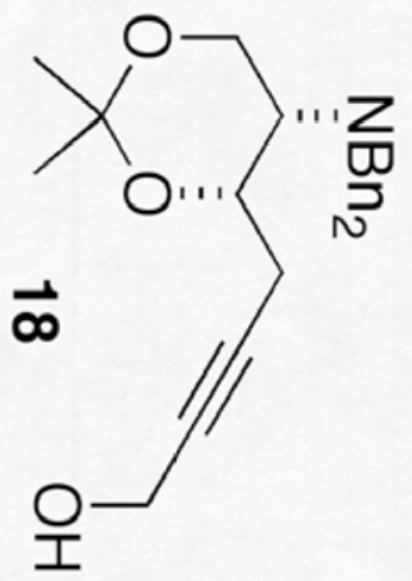

믈 


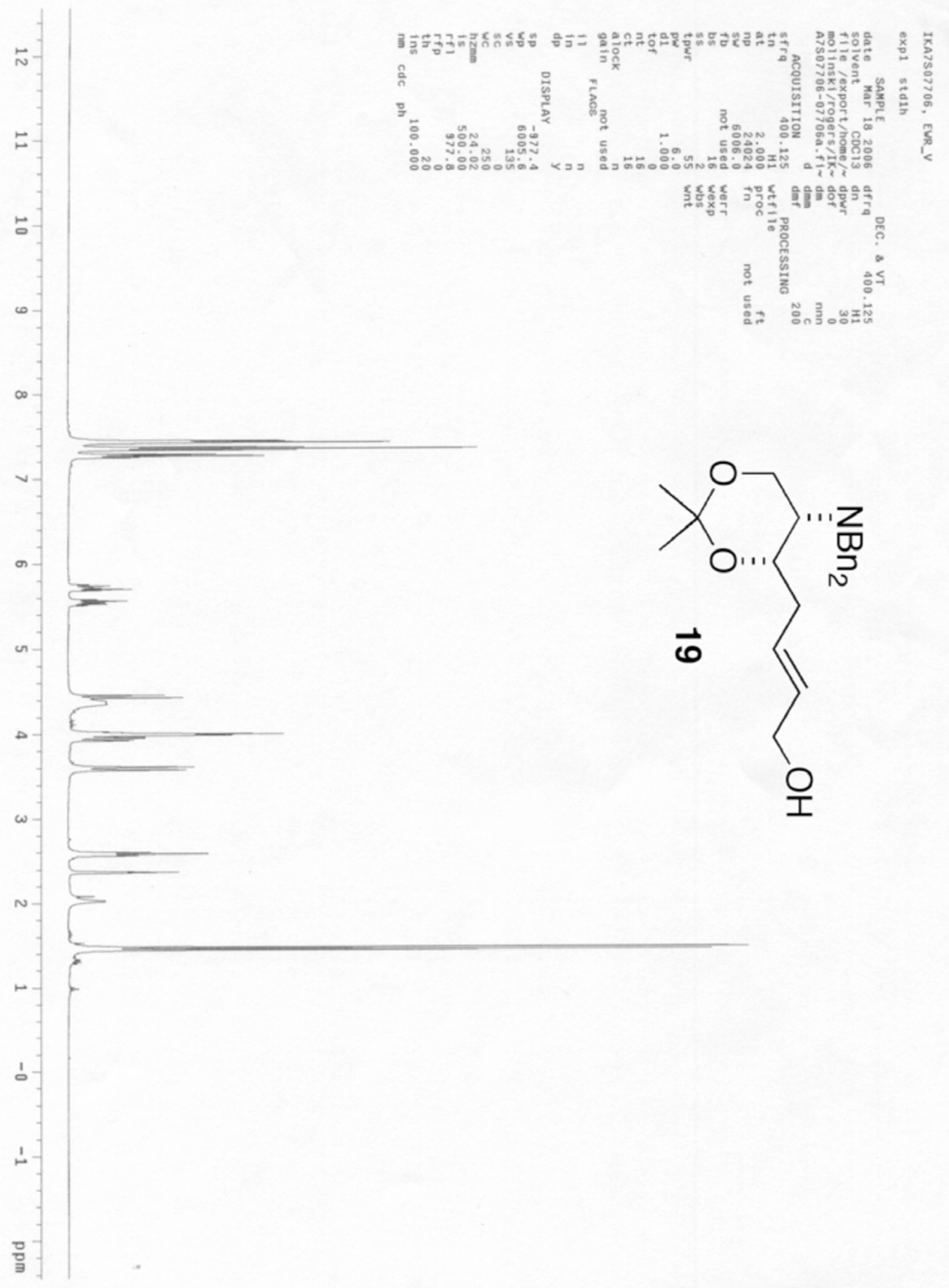




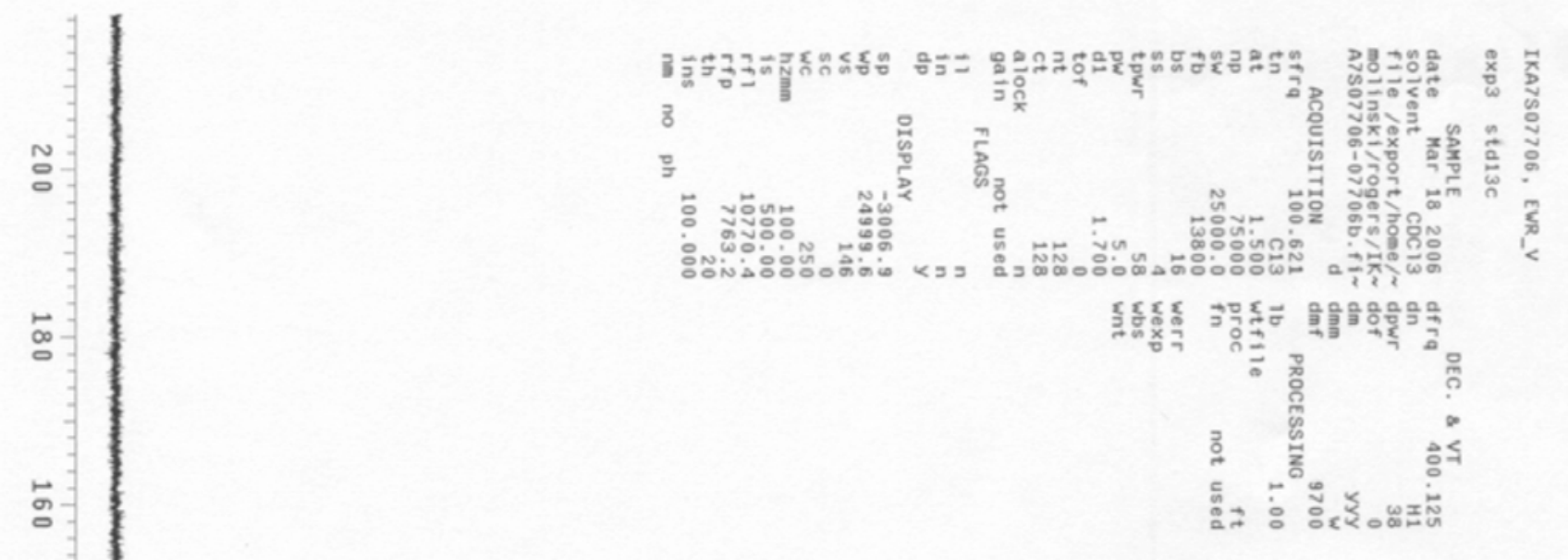

s

:

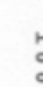$$
\infty
$$$$
\text { . }
$$
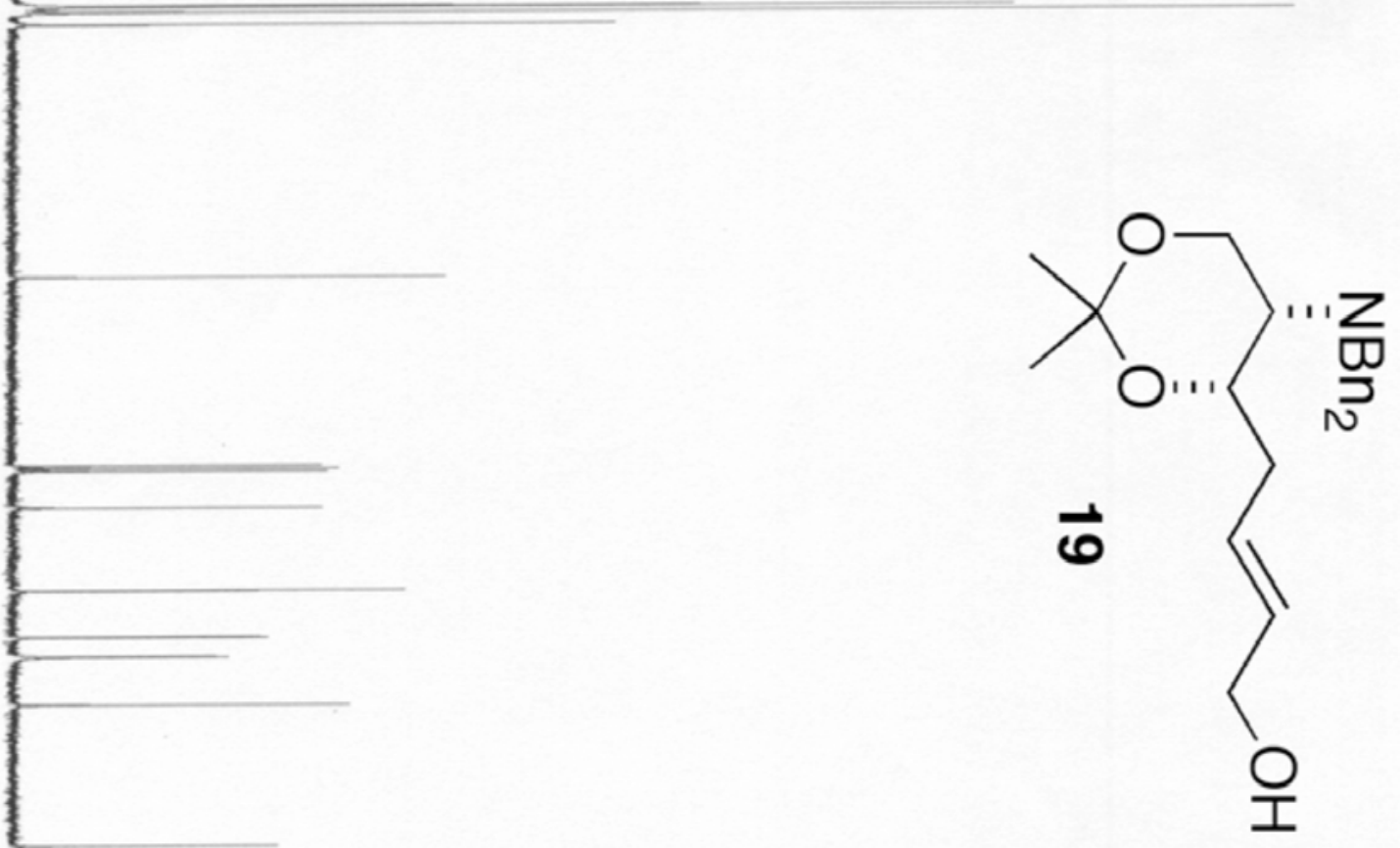



$\circ$

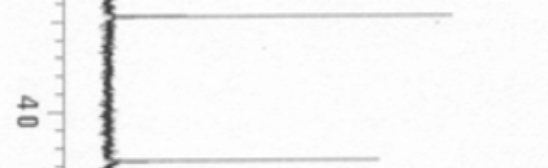

도

-

물 


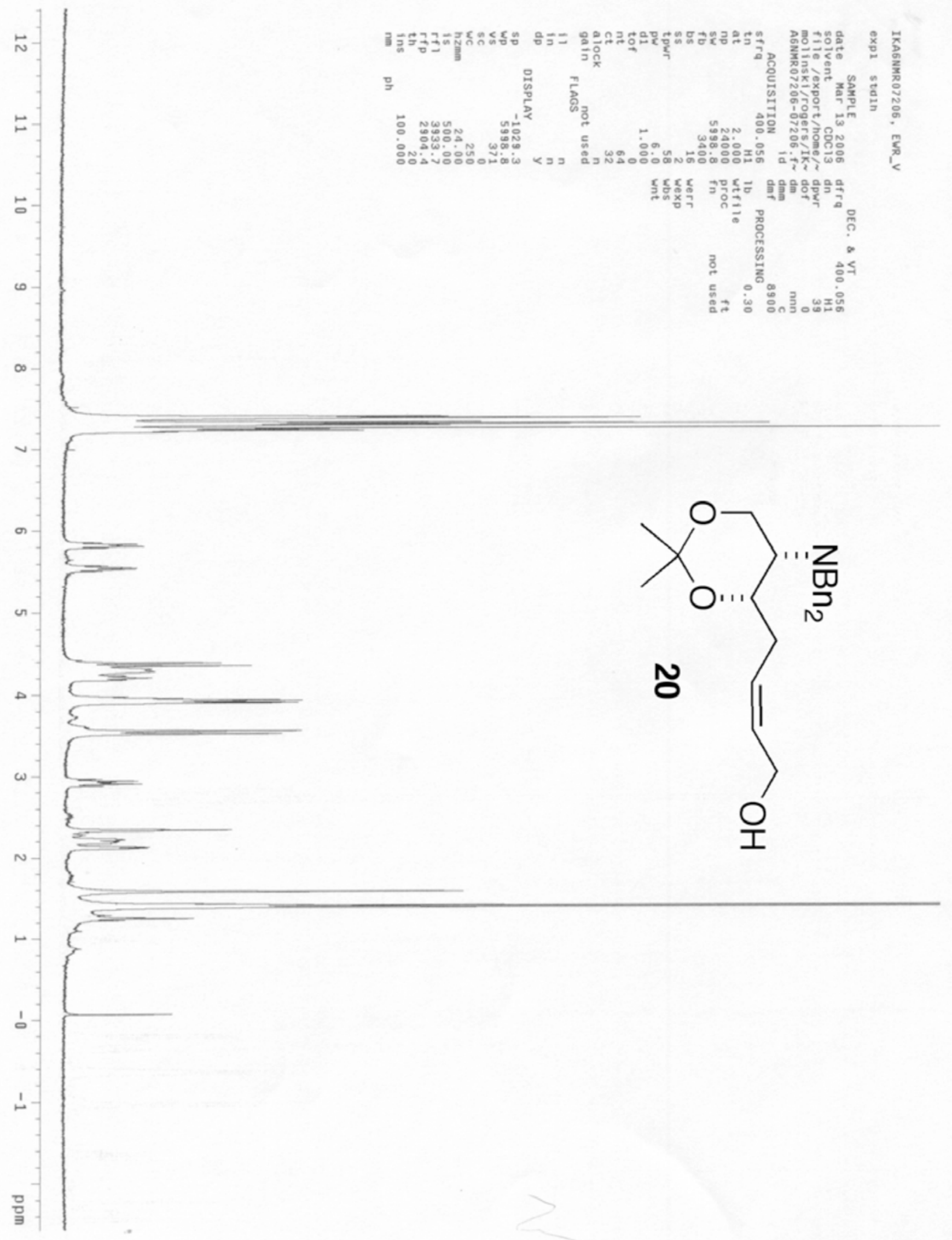




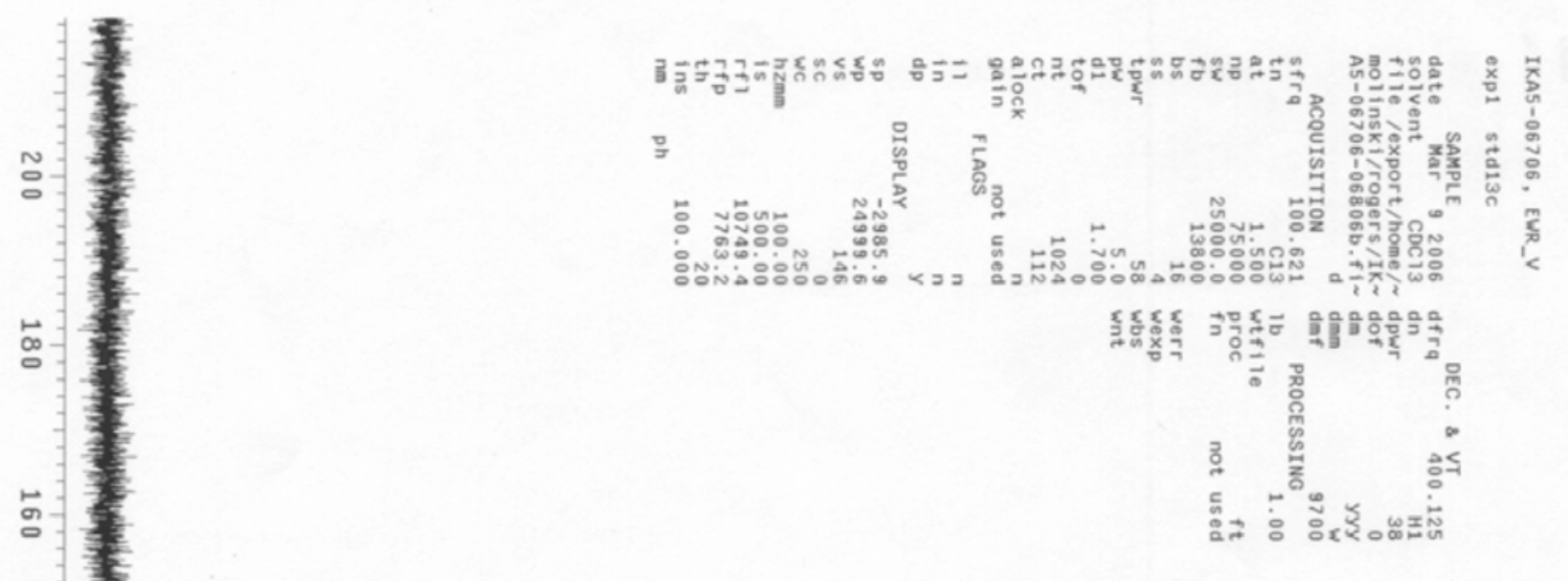

$\stackrel{\circ}{\circ}$

$\stackrel{n}{0}$

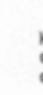

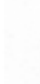

$\stackrel{\infty}{\circ}$

$\circ$

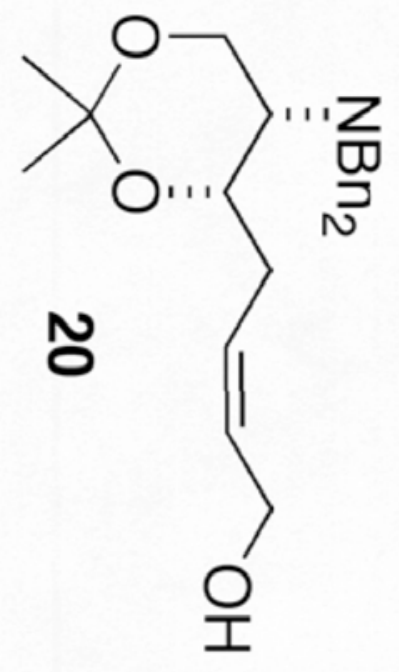

:

ㅇ-

و

언

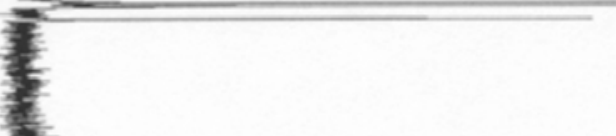

-

$\circ-$

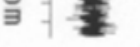




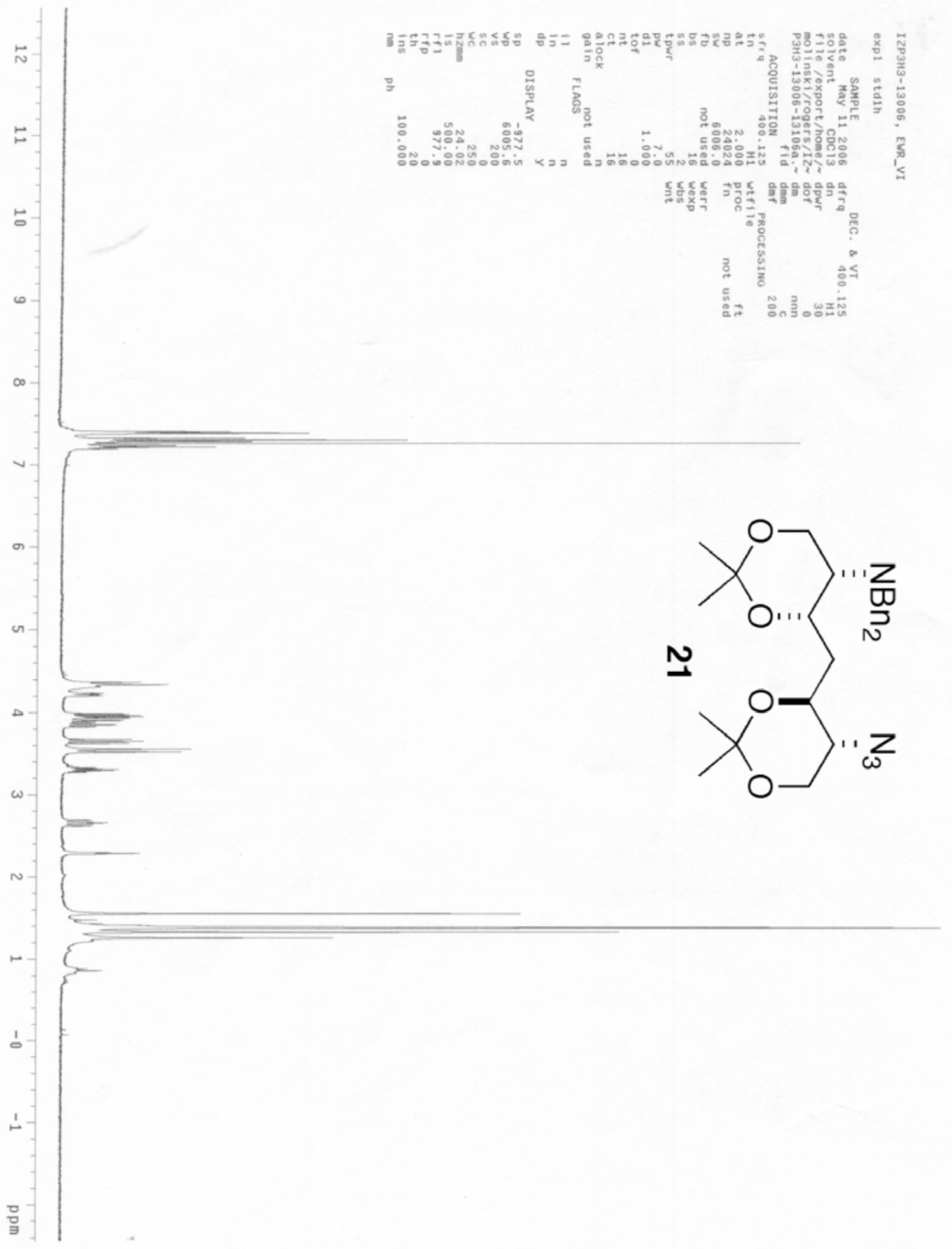




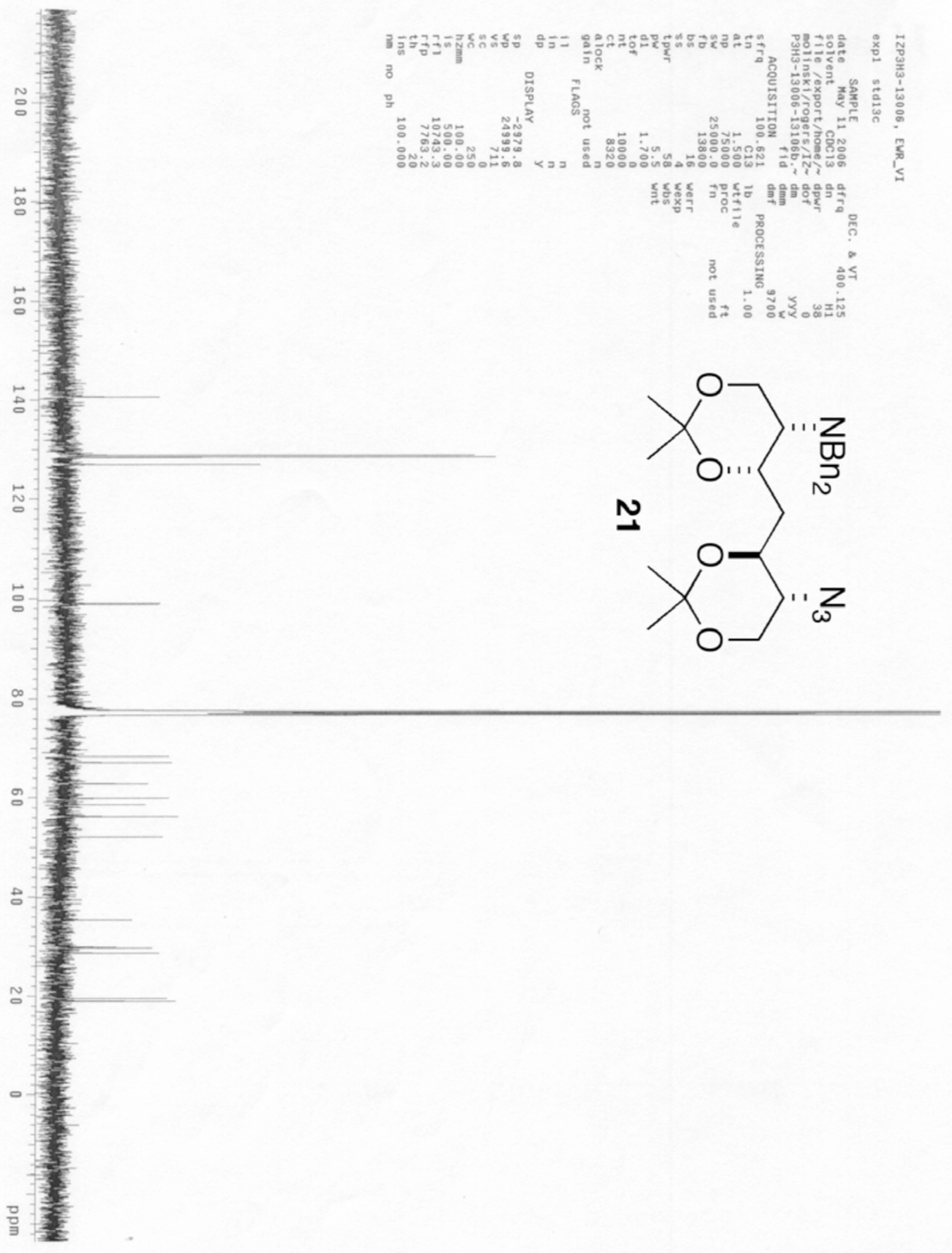




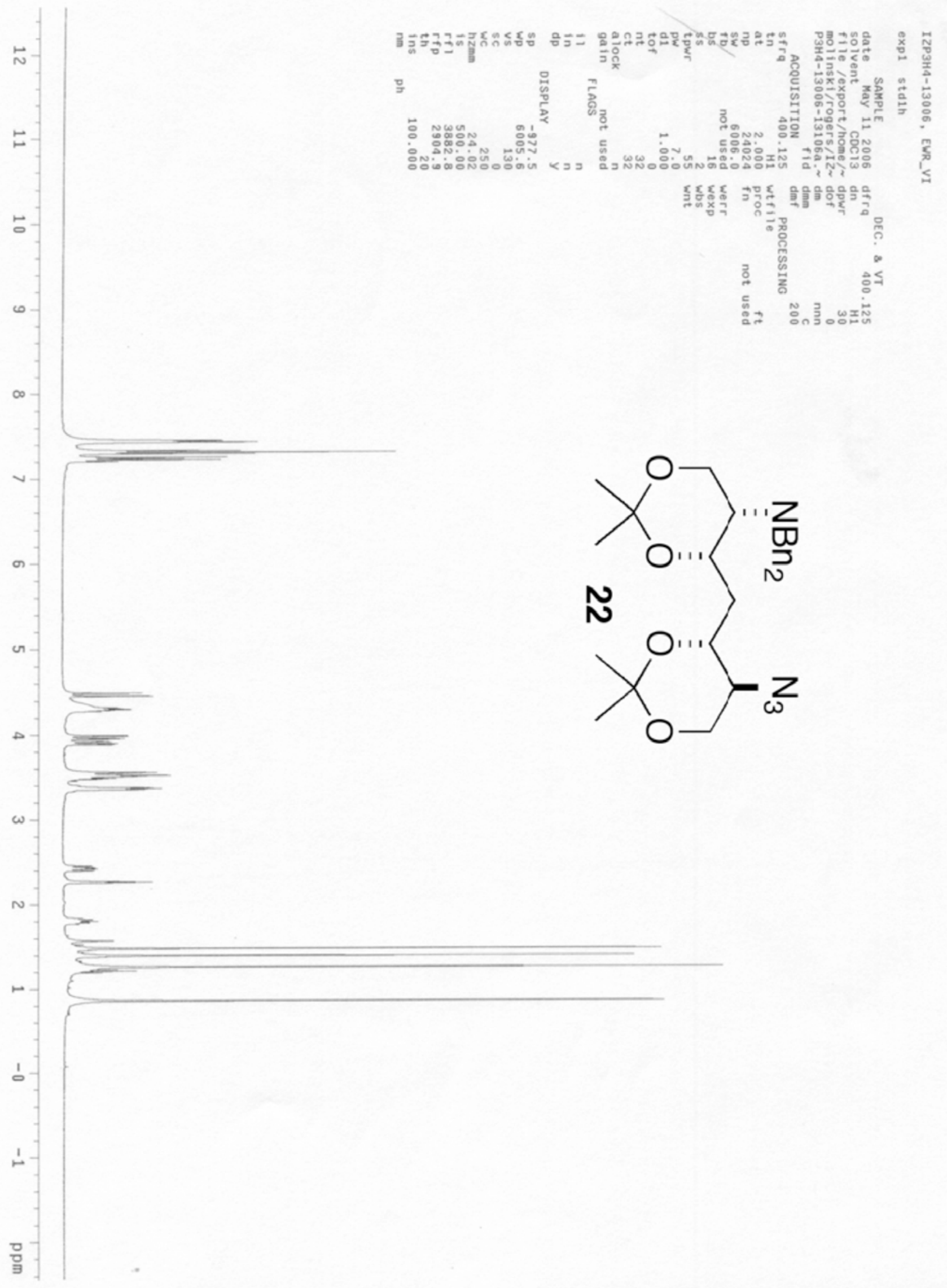



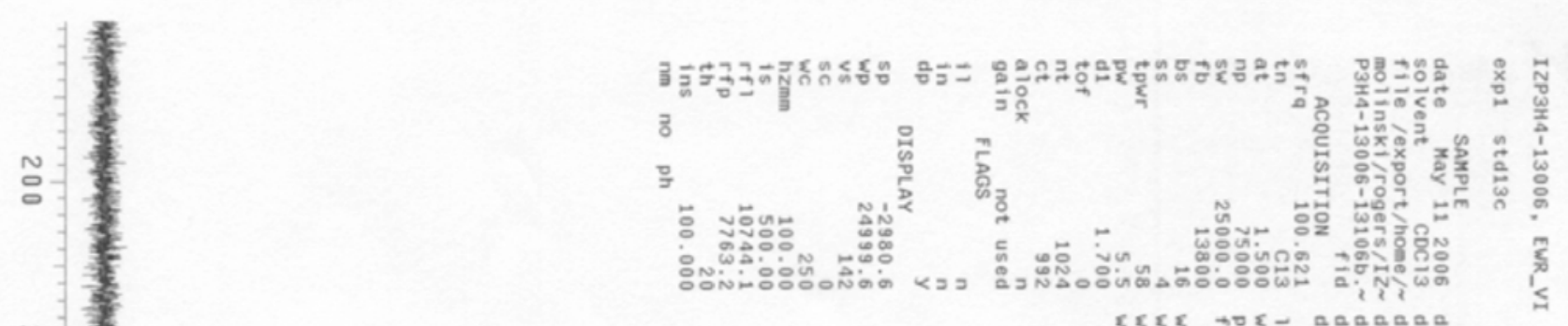

$\stackrel{\infty}{\circ}$

م)

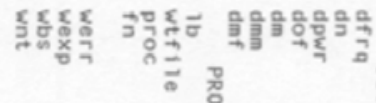

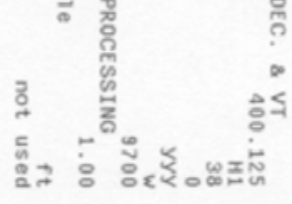

$\stackrel{\bullet}{\circ}$

N
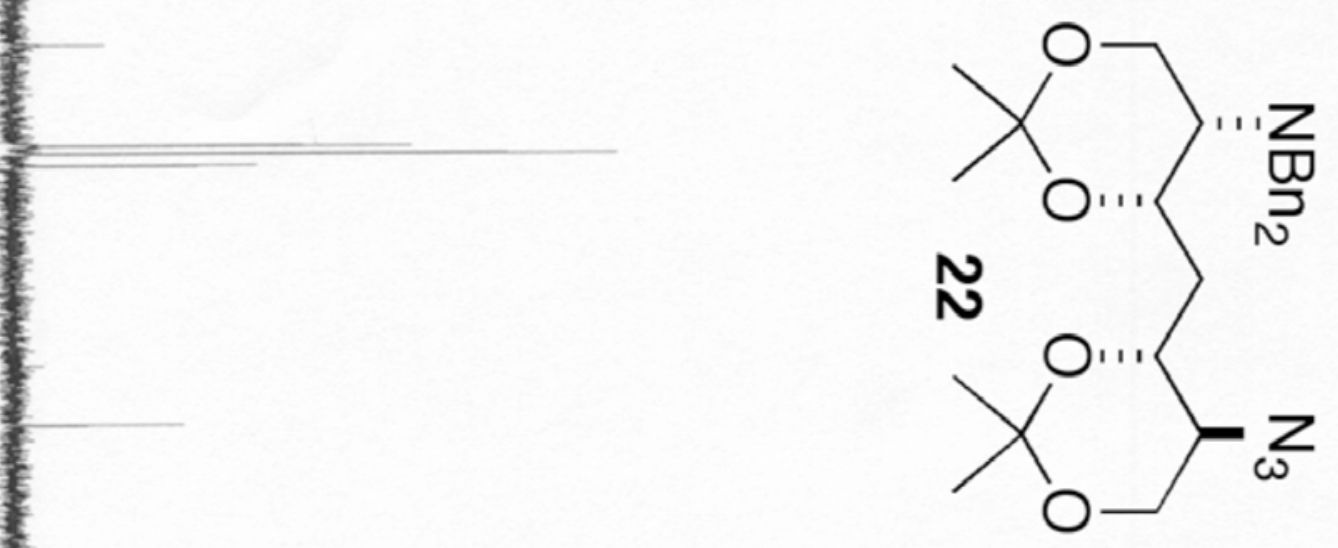

$\infty$

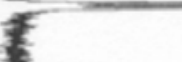

o

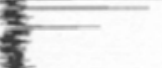

$\stackrel{\circ}{0}$

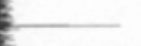

@ 


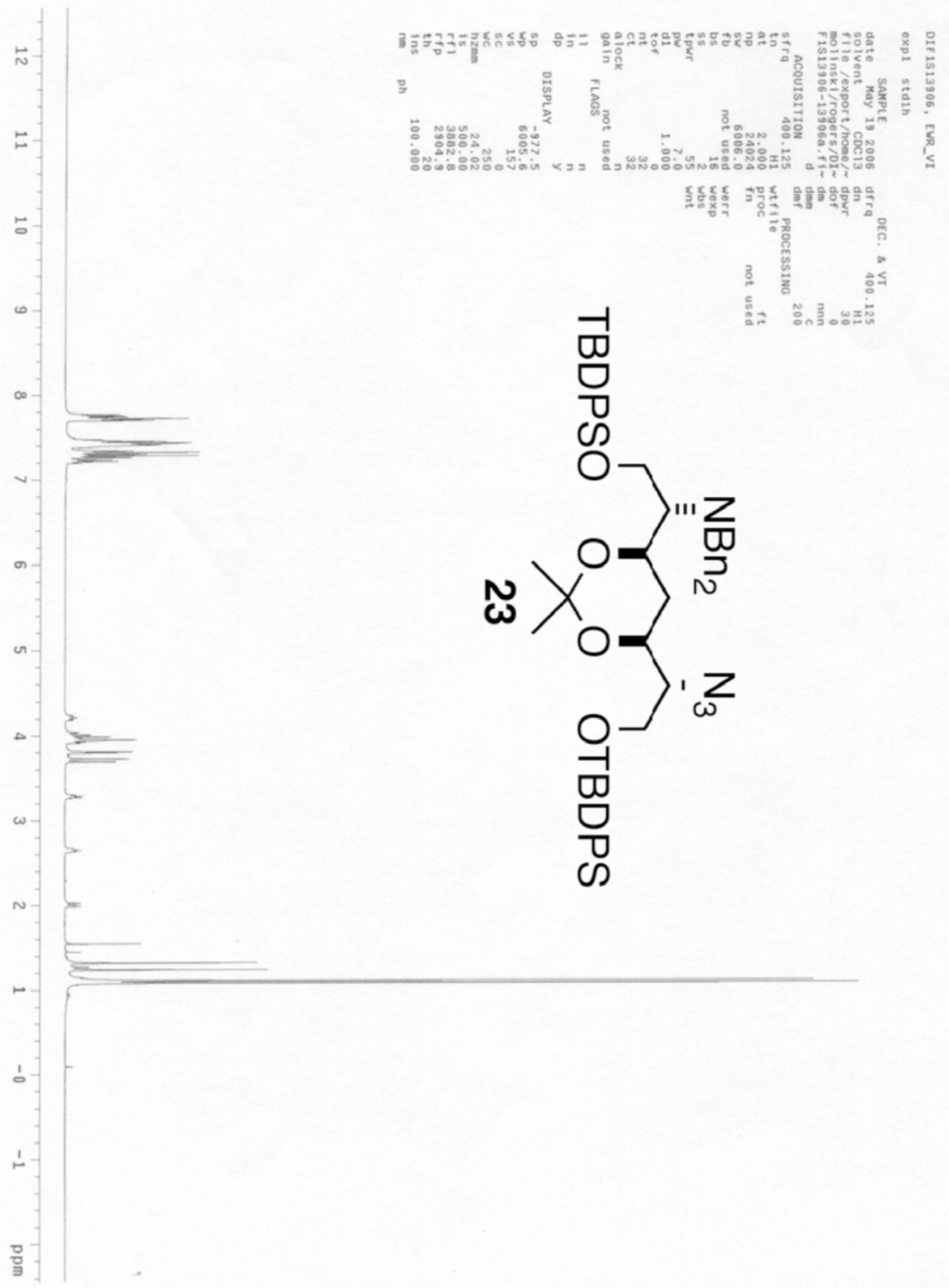




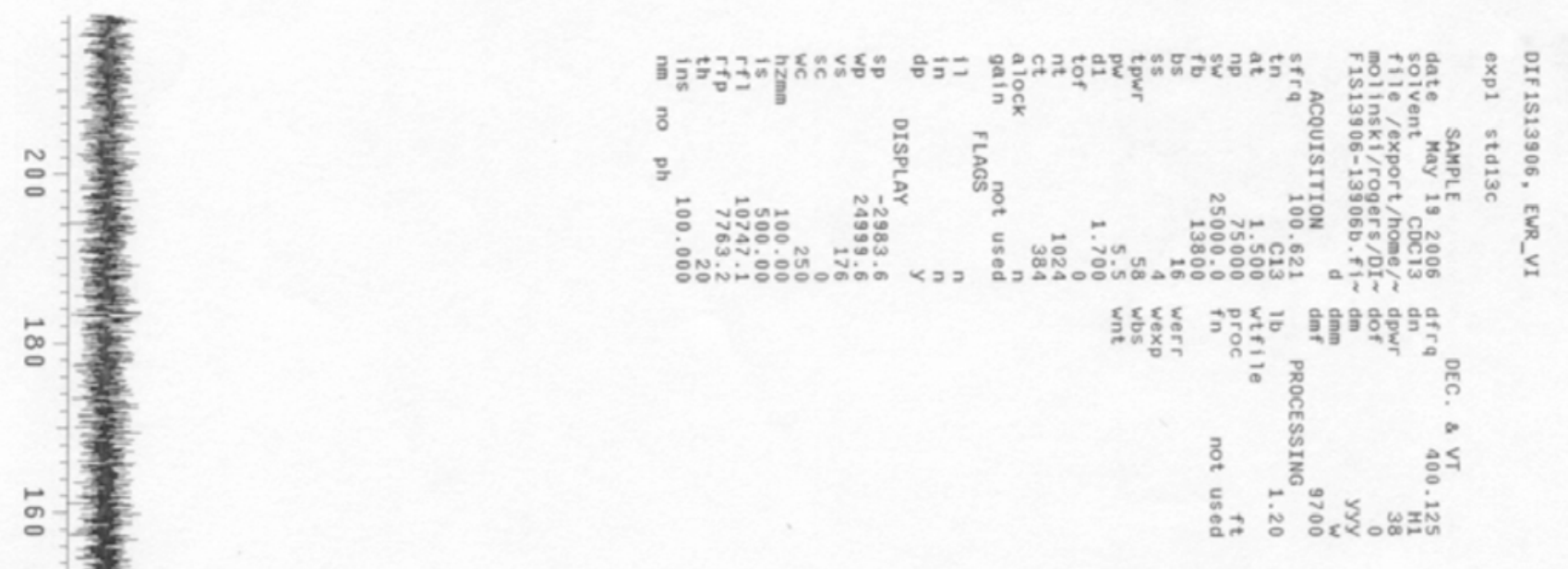

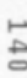

。

.

谙

$-1$

$\infty$

엄

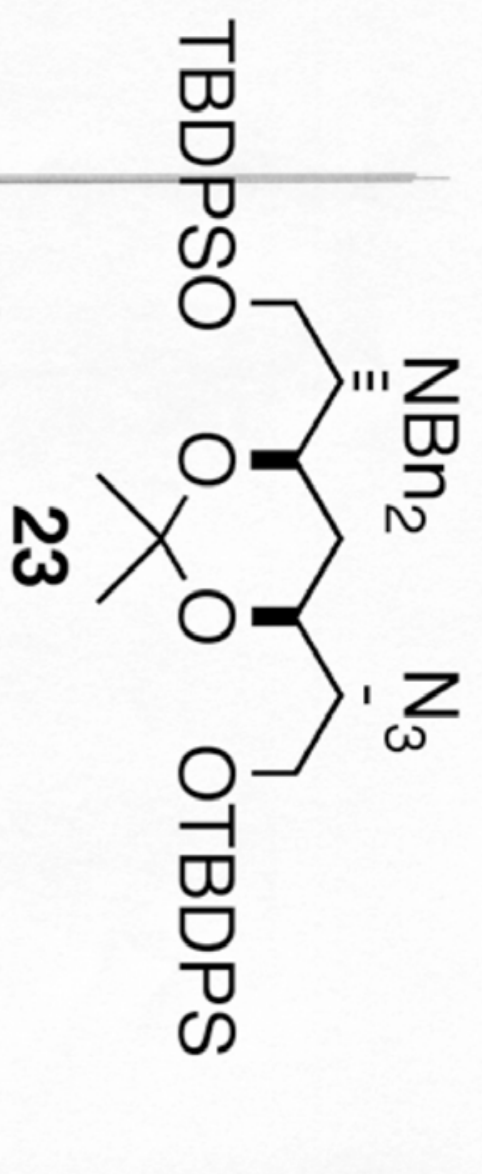

흡 